\title{
Estimates of Dirichlet heat kernel for symmetric Markov processes *
}

\author{
Tomasz Grzywny ${ }^{\dagger}$ Kyung-Youn Kim $\risingdotseq$ Panki Kim ${ }^{\S}$
}

March 6, 2019

\begin{abstract}
We consider a large class of symmetric pure jump Markov processes dominated by isotropic unimodal Lévy processes with weak scaling conditions. First, we establish sharp two-sided heat kernel estimates for these processes in $C^{1,1}$ open sets. As corollaries of our main results, we obtain sharp two-sided Green function estimates and a scale invariant boundary Harnack inequality with explicit decay rates in $C^{1,1}$ open sets.
\end{abstract}

\section{Introduction}

The study of the heat kernel of a semigroup is a field of interactions between probability, analysis and geometry. The transition density function provides direct access to the path properties of a Markov process. In addition, it is the fundamental solution(or heat kernel) of the heat equation with an infinitesimal generator of the corresponding process. The Dirichlet heat kernel describes an operator with zero exterior conditions. For instance, the Green function and the solutions to Cauchy and Poisson problems with Dirichlet conditions are expressed by the heat kernel. In this paper, we consider a large class of symmetric pure jump Markov processes dominated by isotropic unimodal Lévy processes with weak scaling conditions. We estimate the transition density $p_{D}(t, x, y)$ of such Markov processes killed upon leaving an open set $D \subset \mathbb{R}^{d}$ with $C^{1,1}$ smoothness of the boundary. In other words, we establish sharp two-sided estimates of the Dirichlet heat kernel of the integro-differential operators with the maximum principle. Such operators are commonly used to model nonlocal phenomena [32, 8, 37, 28, 30, 22 .

The Dirichlet heat kernel estimates of the Laplacian (the Brownian motion) on the bounded $C^{1,1}$ domains were obtained in [23, 29] (the upper bound) and [45] (the lower bound). See [20] for the Dirichlet heat kernel estimates for more general diffusions, and see [43] for bounds of the Dirichlet heat kernel of the Laplacian on the bounded Lipschitz domain.

For the fractional Laplacian, in 2010, Chen et al [13] gave sharp (two-sided) explicit estimates for the Dirichlet heat kernel $p_{D}(t, x, y)$ of the fractional Laplacian in any $C^{1,1}$ open set $D$

*2010 MSC: Primary 31B25, 60J50; Secondary 60J75, 60J35. Keywords: first exit time, Dirichlet heat kernel, heat kernel, Markov process, transition density, Green function, boundary Harnack inequality

${ }^{\dagger}$ The first author was partially supported by National Science Centre (Poland): grant 2014/14/M/ST1/00600 and the Alexander von Humboldt Foundation

†The second author was partially supported by DAAD PaJaKo Programme and the German Research Foundation (DFG) through the International Research Training Group (IRTG) 2235.

$\S$ This work was supported by the National Research Foundation of Korea(NRF) grant funded by the Korea government(MSIP) (No. NRF-2015R1A4A1041675) 
and over any finite time interval (see [3] for an extension to non-smooth open sets). When $D$ is bounded, large-time Dirichlet heat kernel estimates can be deduced easily from short-time estimates using a spectral analysis.

The approach developed in [13] provides a road map for establishing sharp two-sided Dirichlet heat kernel estimates of other discontinuous processes, and the result has been generalized to more general stochastic processes: purely discontinuous symmetric Lévy processes $([17,5])$, symmetric Lévy processes with Gaussian component ([18]), symmetric non-Lévy processes $([14,33])$ and non-symmetric stable processes with gradient perturbation ([15]).

Let $\mathbb{P}_{y}\left(\tau_{D}>t\right)$ be the survival probability of the corresponding process and $p(t, x, y)=$ $p_{\mathbb{R}^{d}}(t, x, y)$ be the (free) heat kernel for $D=\mathbb{R}^{d}$. Another form of two-sided heat kernel estimates is the following factorization;

$$
c_{1} \mathbb{P}_{x}\left(\tau_{D}>t\right) \mathbb{P}_{y}\left(\tau_{D}>t\right) p(t, x, y) \leqslant p_{D}(t, x, y) \leqslant c_{2} \mathbb{P}_{x}\left(\tau_{D}>t\right) \mathbb{P}_{y}\left(\tau_{D}>t\right) p(t, x, y)
$$

In fact, (1.1) holds for more general sets such as the Lipschitz open set. See [3] 17]. See [6] for a direct approach to obtain sharp estimates of the survival probabilities of unimodal Lévy processes.

Extensions of the result in [13] were obtained for a quite large class of symmetric Lévy processes, including general unimodal Lévy processes with Lévy densities satisfying weak scaling conditions, in [17, 5]. However, the extension to symmetric Markov processes with jumping kernels satisfying similar weak scaling conditions is unknown. In this paper, we extend the results of [5] and [33] to more general processes that are non-isotropic and non-Lévy. Our results cover not only a large class of symmetric Markov processes with jumping kernels satisfying weak scaling conditions, but also a large class of symmetric Markov processes with jumping kernels that decay exponentially with the damping exponent $\beta \in(0, \infty)$, and symmetric finite range Markov processes.

For two non-negative functions $f$ and $g$, the notation $f \asymp g$ means that there are positive constants $c_{1}$ and $c_{2}$ such that $c_{1} g(x) \leqslant f(x) \leqslant c_{2} g(x)$ in the common domain of the definition of $f$ and $g$. We use the symbol ":=," which is read as "is defined to be." For $a, b \in \mathbb{R}$, $a \wedge b:=\min \{a, b\}$ and $a \vee b:=\max \{a, b\}$. We use $d x$ to denote the Lebesgue measure in $\mathbb{R}^{d}$. For a Borel set $A \subset \mathbb{R}^{d}$, we use $|A|$ to denote its Lebesgue measure.

For $0<\underline{\alpha} \leqslant \bar{\alpha}<2$, let $\phi$ be an increasing function on $[0, \infty)$ satisfying that there exist positive constants $\underline{c} \leqslant 1$ and $1 \leqslant \bar{C}$ such that

(WS)

$$
\underline{c}\left(\frac{R}{r}\right)^{\underline{\alpha}} \leqslant \frac{\phi(R)}{\phi(r)} \leqslant \bar{C}\left(\frac{R}{r}\right)^{\bar{\alpha}} \quad \text { for } 0<r \leqslant R .
$$

Using this $\phi$, we define

$$
\nu(r):=\frac{1}{\phi(r) r^{d}} \quad \text { for } r>0 .
$$

Note that according to (WS) and (1.2), there exists $c=c(\bar{\alpha}, \bar{C}, d)$ such that

$$
\nu(r) \leqslant c \nu(2 r) \quad \text { for any } r>0 .
$$

A measure on $\mathbb{R}^{d}$ is called isotropic unimodal, if it is absolutely continuous on $\mathbb{R}^{d} \backslash\{0\}$ with a radial non-increasing density function and a Lévy process is called isotropic unimodal, if the 
one-dimensional distributions are unimodal. Since (WS) implies

$$
\int_{\mathbb{R}^{d}}\left(1 \wedge|x|^{2}\right) \nu(|x|) d x \leqslant c\left(\int_{0}^{1} s^{-\bar{\alpha}+1} d s+\int_{1}^{\infty} s^{-\underline{\alpha}-1}\right) d s<\infty,
$$

$\nu(d x):=\nu(|x|) d x$ is unimodal Lévy measure, and by Proposition in [44] there exists a pure jump isotropic unimodal Lévy process $Z$ corresponding to $\nu$.

Throughout this paper, we assume that $\kappa: \mathbb{R}^{d} \times \mathbb{R}^{d} \rightarrow(0, \infty)$ is a symmetric measurable function and there exists $L_{0}>1$ such that

$$
L_{0}^{-1} \leqslant \kappa(x, y) \leqslant L_{0}, \quad x, y \in \mathbb{R}^{d} .
$$

Let $J: \mathbb{R}^{d} \times \mathbb{R}^{d} \rightarrow(0, \infty)$ be a symmetric measurable function, which is the jumping kernel of our process. We consider two sets of conditions on $J$. The first set is as follows:

(J1) (J1.1) $J(x, y)=\kappa(x, y) \nu(|x-y|)$ on $|x-y| \leqslant 1$,

$(\mathbf{J} 1.2) \sup _{x \in \mathbb{R}^{d}} \int_{|x-y|>1} J(x, y) d y<\infty$

(J1.3) For any $M>0$, there exists $C_{M}>1$ such that $C_{M}^{-1} \nu(|x-y|) \leqslant J(x, y) \leqslant C_{M} \nu(\mid x-$ $y \mid)$ for $|x-y|<M$.

The constant 1 in the condition (J1.1) plays no special role, and it can be changed to any small positive real number. Since (J1.3) implies (J1.1), $J$ satisfies conditions (J1.2) and (J1.3) if and only if $J$ satisfies condition $(\mathbf{J} 1)$.

For the second set of conditions on $J$, let $\chi$ be a non-decreasing function on $(0, \infty)$ with $\chi(r) \equiv \chi(0), r \in(0,1]$, and there exists $\gamma_{1}, \gamma_{2}, L_{1} L_{2}>0$ and $\beta \in[0, \infty]$ such that

$$
L_{1} e^{\gamma_{1} r^{\beta}} \leqslant \chi(r) \leqslant L_{2} e^{\gamma_{2} r^{\beta}}, \quad r>1 .
$$

Then, the second condition on $J$ is as follows:

$$
J(x, y)=\kappa(x, y) \nu(|x-y|) \chi(|x-y|)^{-1}, \quad x, y \in \mathbb{R}^{d},
$$

which is equal to

$$
\begin{cases}\kappa(x, y)\left(\phi(|x-y|)|x-y|^{d} \cdot \chi(|x-y|)\right)^{-1} & \text { if } \beta \in[0, \infty), \\ \kappa(x, y)\left(\phi(|x-y|)|x-y|^{d}\right)^{-1} \mathbf{1}_{\{|x-y| \leqslant 1\}} & \text { if } \beta=\infty .\end{cases}
$$

Clearly (J2) implies (J1.1) and (J1.2). Moreover, if (J2) holds and $\beta \neq \infty$, then (J1) holds.

We consider the Dirichlet form $(\mathcal{E}, \mathcal{F})$ associated with the jumping kernel $J$ :

$$
\mathcal{E}(u, v):=\frac{1}{2} \iint(u(x)-u(y))(v(x)-v(y)) J(x, y) d x d y,
$$

and $\mathcal{F}:=\left\{u \in L^{2}\left(\mathbb{R}^{d}\right): \mathcal{E}(u, u)<\infty\right\}$. Under conditions (J1.1) and (J1.2), according to [40, Theorem 2.1] and [41, Theorem 2.4], $(\mathcal{E}, \mathcal{F})$ is a regular (symmetric) Dirichlet form on $L^{2}\left(\mathbb{R}^{d}, d x\right)$. Moreover, the corresponding Hunt process $Y$ is conservative and $Y$ has the Hölder continuous transition density $p(t, x, y)$ on $(0, \infty) \times \mathbb{R}^{d} \times \mathbb{R}^{d}$ (see [11]).

Now, we state the estimates of the transition density $p(t, x, y)$ of $Y$ with the jumping intensity kernel $J$ satisfying either the conditions (J1.2) and (J1.3), or the condition (J2). The proof of the upper bound of Theorem 1.1 is almost the same as that of [17, (2.6)] using the condition (J1.3) instead of that of [17, (1.5)]. Thus, we skip the proof of the upper bound. The proof of the lower bound is given in Section 6 . 
Theorem 1.1. Suppose that $Y$ is a symmetric pure jump Hunt process whose jumping intensity kernel $J$ satisfies the conditions $(\mathbf{J 1 . 2})$ and (J1.3). Then, for each $M>0$ and $T>0$, there is a positive constant $G \overline{1.1} \geqslant 1$ that depends on $\phi, L_{0}, M$ and $T$ such that for every $(t, x, y) \in(0, T] \times \mathbb{R}^{d} \times \mathbb{R}^{d}$ with $|x-y|<M$, the function $p(t, x, y)$ has the following estimates:

$$
C_{[1.1}^{-1}\left(\left[\phi^{-1}(t)\right]^{-d} \wedge t \nu(|x-y|)\right) \leqslant p(t, x, y) \leqslant C_{1.1}\left(\left[\phi^{-1}(t)\right]^{-d} \wedge t \nu(|x-y|)\right)
$$

where $\phi^{-1}(t)$ is the inverse function of $\phi(t)$.

For notational convenience, for each $a, \gamma, T>0$, we define a function $F_{a, \gamma, T}(t, r)$ on $(0, T] \times$ $[0, \infty)$ as

$$
F_{a, \gamma, T}(t, r):= \begin{cases}{\left[\phi^{-1}(t)\right]^{-d} \wedge t \nu(r) e^{-\gamma r^{\beta}}} & \text { if } \beta \in[0,1], \\ {\left[\phi^{-1}(t)\right]^{-d} \wedge t \nu(r)} & \text { if } \beta \in(1, \infty] \text { with } r<1, \\ t \exp \left\{-a\left(r\left(\log \frac{T r}{t}\right)^{\frac{\beta-1}{\beta}} \wedge r^{\beta}\right)\right\} & \text { if } \beta \in(1, \infty) \text { with } r \geqslant 1, \\ (t /(T r))^{a r}=\exp \left\{-\operatorname{ar}\left(\log \frac{T r}{t}\right)\right\} & \text { if } \beta=\infty \text { with } r \geqslant 1 .\end{cases}
$$

Theorem 1.2. Suppose that $Y$ is a symmetric pure jump Hunt process whose jumping intensity kernel J satisfies the condition (J2). Then, the process $Y$ has a continuous transition density function $p(t, x, y)$ on $(0, \infty) \times \mathbb{R}^{d} \times \mathbb{R}^{d}$. For each $T>0$, there are positive constants $Q_{1.2} \geqslant 1, c_{1}$ and $c_{2} \geqslant 1$ that depend on $\phi, L_{0}, \beta, \chi$ and $T$ such that for every $t \in(0, T]$ the function $p(t, x, y)$ has the following estimates:

$$
c_{2}^{-1} F_{c_{1}, \gamma_{2}, T}(t,|x-y|) \leqslant p(t, x, y) \leqslant c_{2} F_{q_{1.2}^{\gamma_{1}, T}}(t,|x-y|) .
$$

Theorem 1.2 for the case $\beta \in(1, \infty]$ is basically derived from [12, Theorems 1.2 and 1.4]. Despite using 1 instead of $T$, the proof is the same. When $\beta \in[0,1]$, the upper bound in Theorem [1.2 is derived from [31, Theorem 2, Proposition 1]. The lower bound in Theorem 1.2 is proved as a special case of the preliminary lower bound on the heat kernel of the killed process in Section 6.

For any open set $D \subset \mathbb{R}^{d}$, the first exit time of $D$ by process $Y$ is defined by the formula $\tau_{D}:=\inf \left\{t>0: Y_{t} \notin D\right\}$ and we use $Y^{D}$ to denote the process obtained by killing process $Y$ upon exiting $D$. The strong Markov property is used to easily verify that $p_{D}(t, x, y):=$ $p(t, x, y)-\mathbb{E}_{x}\left[p\left(t-\tau_{D}, Y_{\tau_{D}}, y\right) ; t>\tau_{D}\right]$ is the transition density of $Y^{D}$. Using the continuity and estimate of $p$, it is routine to show that $p_{D}(t, x, y)$ is symmetric and continuous (e.g., see the proof of Theorem 2.4 in [21]).

Let $D \subset \mathbb{R}^{d}$ (when $d \geqslant 2$ ) be a $C^{1,1}$ open set, that is, there exists a localization radius $R_{0}>0$ and a constant $\Lambda>0$ such that for every $z \in \partial D$ there exists a $C^{1,1}$-function $\varphi=\varphi_{z}: \mathbb{R}^{d-1} \rightarrow \mathbb{R}$ satisfying $\varphi(0)=0, \nabla \varphi(0)=(0, \ldots, 0),\|\nabla \varphi\|_{\infty} \leqslant \Lambda,|\nabla \varphi(x)-\nabla \varphi(w)| \leqslant \Lambda|x-w|$ and an orthonormal coordinate system $C S_{z}$ of $z=\left(z_{1}, \cdots, z_{d-1}, z_{d}\right):=\left(\widetilde{z}, z_{d}\right)$ with an origin at $z$ such that $D \cap B\left(z, R_{0}\right)=\left\{y=\left(\tilde{y}, y_{d}\right) \in B\left(0, R_{0}\right)\right.$ in $\left.C S_{z}: y_{d}>\varphi(\widetilde{y})\right\}$. The pair $\left(R_{0}, \Lambda\right)$ is called the $C^{1,1}$ characteristics of the open set $D$. Note that a $C^{1,1}$ open set $D$ with characteristics $\left(R_{0}, \Lambda\right)$ can be unbounded and disconnected, and the distance between two distinct components of $D$ is at least $R_{0}$. By a $C^{1,1}$ open set in $\mathbb{R}$ with a characteristic $R_{0}>0$, we mean an open set that can be written as the union of disjoint intervals so that the infimum of the lengths of all these intervals is at least $R_{0}$ and the infimum of the distances between these intervals is at least $R_{0}$.

It is well known that if $D$ is $C^{1,1}$ open set with the characteristics $\left(R_{0}, \Lambda\right)$, then $D$ satisfies the interior and exterior ball conditions with the characteristic $R_{1} \leqslant R_{0}$. That is, there exist 
balls $B_{1}, B_{2} \subset \mathbb{R}^{d}$ with radius $R_{1}$ such that $B_{1} \subset D \subset B_{2}^{c}$ satisfying $\delta_{B_{1}}(x)=\delta_{D}(x)=\delta_{B_{2}}(x)$ for any $x \in B_{1}$. Throughout this paper, without loss of generality, we always assume that $R_{0}=R_{1}$.

To obtain the sharp estimates of the exit distributions for $Y$ (see Theorem 4.2), we need additional conditions for the regularity of $\kappa$ and $\phi$.

$\left(\mathbf{K}_{\eta}\right)$ There are $L_{3}>0$ and $\eta>\bar{\alpha} / 2$ such that $|\kappa(x, x+h)-\kappa(x, x)| \leqslant L_{3}|h|^{\eta}$ for every $x, h \in \mathbb{R}^{d},|h| \leqslant 1$.

Note that the condition $\left(\mathbf{K}_{\eta}\right)$ implies that

$$
\left|\kappa\left(x+h_{1}, x+h_{2}\right)-\kappa(x, x)\right| \leqslant 2 L_{3}\left(\left|h_{1}\right|^{\eta}+\left|h_{2}\right|^{\eta}\right), \quad \text { for }\left|h_{1}\right|,\left|h_{2}\right|<1 .
$$

(SD) $\phi \in C^{1}(0, \infty)$ and $r \rightarrow-\nu^{\prime}(r) / r$ is decreasing.

(See Remark 1.4.)

Now, we state the following theorem, which is one of the main results of this paper. Let $\delta_{D}(x)$ be the distance between $x$ and $D^{c}$, and let

$$
\Psi(t, x):=\left(1 \wedge \sqrt{\frac{\phi\left(\delta_{D}(x)\right)}{t}}\right)
$$

Theorem 1.3. Suppose that $Y$ is a symmetric pure jump Hunt process whose jumping intensity kernel $J$ satisfies the conditions $(\mathbf{J 1}),(\mathbf{S D})$, and $\left(\mathbf{K}_{\eta}\right)$. Suppose that $D$ is a bounded $C^{1,1}$ open set in $\mathbb{R}^{d}$ with characteristics $\left(R_{0}, \Lambda\right)$. Then, for each $T>0$, there exists $c_{1}=$ $c_{1}\left(\phi, L_{0}, L_{3}, \eta, R_{0}, \Lambda, T, d\right)$ and $c_{2}=c_{2}\left(\phi, L_{0}, L_{3}, \eta, R_{0}, \Lambda, T, d, \operatorname{diam}(D)\right)>0$ such that the transition density $p_{D}(t, x, y)$ of $Y^{D}$ has the following estimates.

(1) For any $(t, x, y) \in(0, T] \times D \times D$, we have

$$
c_{1}^{-1} \Psi(t, x) \Psi(t, y) p(t, x, y) \leqslant p_{D}(t, x, y) \leqslant c_{1} \Psi(t, x) \Psi(t, y) p(t, x, y)
$$

(2) For any $(t, x, y) \in[T, \infty) \times D \times D$, we have

$$
c_{2}^{-1} e^{-t \lambda^{D}} \sqrt{\phi\left(\delta_{D}(x)\right)} \sqrt{\phi\left(\delta_{D}(y)\right)} \leqslant p_{D}(t, x, y) \leqslant c_{2} e^{-t \lambda^{D}} \sqrt{\phi\left(\delta_{D}(x)\right)} \sqrt{\phi\left(\delta_{D}(y)\right)},
$$

where $-\lambda^{D}<0$ is the largest eigenvalue of the generator of $Y^{D}$.

Remark 1.4. Conditions (SD) and (WS) hold for a large class of pure jump isotropic unimodal Lévy processes including all subordinate Brownian motions with weak scaling conditions (see (1.8)): let $W=\left(W_{t}, \mathbb{P}_{x}\right)$ be a Brownian motion in $\mathbb{R}^{d}$ and $S=\left(S_{t}\right)$ be an independent driftless subordinator with Laplace exponent $\varphi_{1}$. The Laplace exponent $\varphi_{1}$ is a Bernstein function with $\varphi_{1}(0+)=0$. Since $\varphi_{1}$ has no drift part, $\varphi_{1}$ can be written in the form

$$
\varphi_{1}(\lambda)=\int_{0}^{\infty}\left(1-e^{-\lambda t}\right) \mu(d t) .
$$

Here $\mu$ is a $\sigma$-finite measure on $(0, \infty)$ satisfying $\int_{0}^{\infty}(t \wedge 1) \mu(d t)<\infty$. $\mu$ is called the Lévy measure of the subordinator $S$. 
The subordinate Brownian motion $Z=\left(Z_{t}, \mathbb{P}_{x}\right)$ is defined by $Z_{t}=W_{S_{t}}$. The density of the Lévy measure of $Z$ with respect to the Lebesgue measure is given by $x \rightarrow \nu_{d}(|x|)$ with

$$
\nu_{d}(r)=\int_{0}^{\infty}(4 \pi t)^{-d / 2} \exp \left(-\frac{r^{2}}{4 t}\right) \mu(t) d t, \quad r \neq 0 .
$$

Thus, $r \rightarrow \nu_{d}(r)$ is smooth for $r>0$, and

$$
-\frac{\nu_{d}^{\prime}(r)}{r}=2 \pi \nu_{d+2}(r), \quad r>0
$$

which is decreasing. Suppose that

$$
\underline{c}\left(\frac{R}{r}\right)^{\underline{\alpha} / 2} \leqslant \frac{\varphi_{1}(R)}{\varphi_{1}(r)} \leqslant \bar{C}\left(\frac{R}{r}\right)^{\bar{\alpha} / 2} \quad \text { for } 0<r \leqslant R .
$$

Then, by [4, Theorem 26]

$$
\nu_{d}(r) \asymp r^{-d} \varphi_{1}\left(r^{-2}\right) .
$$

Let $\widehat{\phi}(r):=r^{-d} \nu_{d}(r)^{-1}$ (so that $\left.\nu_{d}(r)=\widehat{\phi}(r)^{-1} r^{-d}\right)$. Then $\widehat{\phi}$ is smooth, and since $\widehat{\phi}(r) \asymp$ $\varphi_{1}\left(r^{-2}\right)^{-1}$, it satisfies (WS).

When either $D$ is unbounded or $\beta=\infty$, we need precise information on $J$, which is encoded in (J2), for large $|x-y|$. Moreover, when $\beta \in(1, \infty]$, we need to impose an addition assumption for $D$ in order to obtain the sharp lower bound of $p_{D}(t, x, y)$; We say that the path distance in an open set $U$ is comparable to the Euclidean distance with characteristic $\lambda_{1}$ if for every $x$ and $y$ in $U$ there is a rectifiable curve $l$ in $U$ that connects $x$ to $y$ such that the length of $l$ is less than or equal to $\lambda_{1}|x-y|$. Clearly, such a property holds for all bounded $C^{1,1}$ connected open sets, $C^{1,1}$ connected open sets with compact complements, and connected open sets above graphs of $C^{1,1}$ functions.

Theorem 1.5. Suppose that $Y$ is a symmetric pure jump Hunt process whose jumping intensity kernel $J$ satisfies the conditions $(\mathbf{J} 2),(\mathbf{S D})$ and $\left(\mathbf{K}_{\eta}\right)$. Suppose that $D$ is a $C^{1,1}$ open set in $\mathbb{R}^{d}$ with characteristics $\left(R_{0}, \Lambda\right)$. Then, for each $T>0$, the transition density $p_{D}(t, x, y)$ of $Y^{D}$ has the following estimates.

(1) There is a positive constant $c_{1}=c_{1}\left(\beta, \chi, \phi, L_{0}, L_{3}, \eta, R_{0}, \Lambda, T, d\right)$ such that for all $(t, x, y) \in$ $(0, T] \times D \times D$ we have

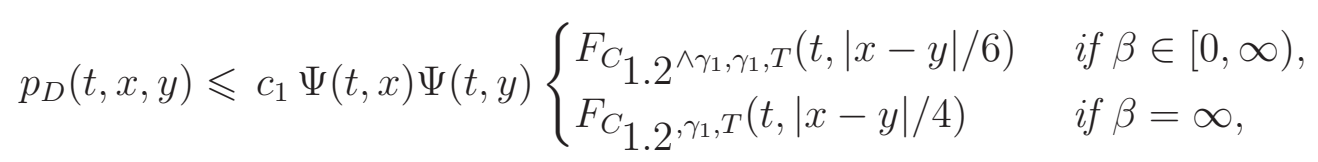

where $C_{1.2}$ is the constant in Theorem 1.2.

(2) There is a positive constant $c_{2}=c_{2}\left(\beta, \chi, \phi, L_{0}, L_{3}, \eta, R_{0}, \Lambda, T, d\right)$ such that for all $t \in(0, T]$ we have

$$
p_{D}(t, x, y) \geqslant c_{2} \Psi(t, x) \Psi(t, y) \begin{cases}{\left[\phi^{-1}(t)\right]^{-d} \wedge t e^{-\gamma_{2}|x-y|^{\beta}} \nu(|x-y|)} & \text { if } \beta \in[0,1], \\ {\left[\phi^{-1}(t)\right]^{-d} \wedge t \nu(|x-y|)} & \text { if } \beta \in(1, \infty) \text { and }|x-y|<1,\end{cases}
$$


(3) Suppose, in addition, that the path distance in $D$ is comparable to the Euclidean distance with characteristic $\lambda_{1}$. Then, there are positive constants $c_{i}=c_{i}\left(\beta, \chi, \phi, L_{0}, L_{3}, \eta, R_{0}, \Lambda, T, d, \lambda_{1}\right)$, $i=3,4$, such that if $x, y \in D$ and $t \in(0, T]$, we have

$$
p_{D}(t, x, y) \geqslant c_{3} \Psi(t, x) \Psi(t, y) \begin{cases}F_{c_{4}, \gamma_{2}, T}(t,|x-y|) & \text { if } \beta \in(1, \infty) \text { and }|x-y| \geqslant 1, \\ F_{c_{4}, \gamma_{2}, T}(t, 5|x-y| / 4) & \text { if } \beta=\infty \text { and }|x-y| \geqslant 4 / 5 .\end{cases}
$$

(4) If $\beta \in(1, \infty)$, there is a positive constant $c_{5}=c_{5}\left(\beta, \chi, \phi, L_{0}, L_{3}, \eta, R_{0}, \Lambda, T, d\right)$ such that for every $x, y$ in the different components of $D$ with $|x-y| \geqslant 1$ and $t \in(0, T]$ we have

$$
p_{D}(t, x, y) \geqslant c_{5} \Psi(t, x) \Psi(t, y) t e^{-\gamma_{2}(5|x-y| / 4)^{\beta}} \nu(|x-y|) .
$$

(5) Suppose in addition that $\beta=\infty$ and $D$ is bounded and connected. Then the claim of Theorem 1.3 (2) holds.

Recall that the Green function $G_{D}(x, y)$ of $Y$ on $D$ is defined as $G_{D}(x, y)=\int_{0}^{\infty} p_{D}(t, x, y) d t$. As an application of Theorem 1.3 and [1.5, we derive the sharp two sided estimate on the Green function $G_{D}(x, y)$ of $Y$ on bounded $C^{1,1}$ open sets. For notational convenience, let

$$
a(x, y):=\sqrt{\phi\left(\delta_{D}(x)\right)} \sqrt{\phi\left(\delta_{D}(y)\right)}
$$

and

$$
g(x, y):= \begin{cases}\frac{\phi(|x-y|)}{|x-y|^{d}}\left(1 \wedge \frac{\phi\left(\delta_{D}(x)\right)}{\phi(|x-y|)}\right)^{1 / 2}\left(1 \wedge \frac{\phi\left(\delta_{D}(y)\right)}{\phi(|x-y|)}\right)^{1 / 2} & \text { when } d \geqslant 2, \\ \frac{a(x, y)}{|x-y|} \wedge\left(\frac{a(x, y)}{\phi^{-1}(a(x, y))}+\left(\int_{|x-y|}^{\phi^{-1}(a(x, y))} \frac{\phi(s)}{s^{2}} d s\right)^{+}\right) & \text {when } d=1\end{cases}
$$

where $x^{+}:=x \vee 0$.

Theorem 1.6. Suppose that $D$ is a bounded $C^{1,1}$ open set in $\mathbb{R}^{d}$ with characteristics $\left(R_{0}, \Lambda\right)$. Let $Y$ be a symmetric pure jump Hunt process whose jumping intensity kernel $J$ satisfies $\left(\mathbf{K}_{\eta}\right)$ and (SD). Suppose either (1) the jumping intensity kernel $J$ satisfies the condition (J1), or (2) $D$ is connected and the jumping intensity kernel $J$ satisfies the condition (J2) with $\beta=\infty$. Then for every $(x, y) \in D \times D$, we have $G_{D}(x, y) \asymp g(x, y)$.

Remark 1.7. When $d=1$, if either $\underline{\alpha}<1$ or $\bar{\alpha}>1$, one can write the Green function estimates in simpler forms. (see, [17, Corollary 7.4 and Remark 7.5])

In addition, we obtain the uniform and scale-invariant boundary Harnack inequality with explicit decay rates in $C^{1,1}$ open sets as an application of Theorems 1.5 and 1.6(1). A function $f: \mathbb{R}^{d} \rightarrow \mathbb{R}$ is said to be harmonic in the open set $D$ with respect to $Y$ if for every open set $U \subset D$ whose closure is a compact subset of $D, \mathbb{E}_{x}\left[|f|\left(Y_{\tau_{U}}\right)\right]<\infty$ for every $x \in U$ and

$$
f(x)=\mathbb{E}_{x}\left[f\left(Y_{\tau_{U}}\right)\right] \quad \text { for every } x \in U .
$$

It is said that $f$ is regular harmonic in $D$ with respect to $Y$ if $f$ is harmonic in $D$ with respect to $Y$ and (1.10) holds for $U=D$.

The next condition guarantees that $C_{c}^{2}\left(\mathbb{R}^{d}\right)$ is in the domain of the Feller generator. 
(L) $Y$ is Feller and there exists a function $q(r)$ such that $J(x, y) \leqslant q(|x-y|)$ and

$$
\lim _{R \rightarrow \infty} \int_{|h|>R} q(|h|) d h=0 .
$$

Note that if (J2) holds, then clearly (1.11) holds, and $Y$ is Feller based on Theorem 1.2, Thus, condition $(\mathrm{L})$ is weaker than condition $(\mathrm{J} 2)$.

The next condition on $J$ is necessary for the boundary Harnack inequality to hold (see [7, Assumption C and Example 5.14]).

(C) For any $0<r<R \leqslant 2$ there exists $C^{*}=C^{*}(\phi, d, r / R)$ such that for any $x_{0} \in \mathbb{R}^{d}$, $x \in B\left(x_{0}, r\right)$ and $y \in B\left(x_{0}, R\right)^{c},\left(C^{*}\right)^{-1} J\left(x_{0}, y\right) \leqslant J(x, y) \leqslant C^{*} J\left(x_{0}, y\right)$.

Note that when $\beta \in[0,1]$, condition (J2) implies (C). In contrast, when $\beta \in(1, \infty]$, the boundary Harnack inequality does not hold under condition (J2).

Theorem 1.8. Suppose $D$ is a $C^{1,1}$ open set in $\mathbb{R}^{d}$ with characteristics $\left(R_{0}, \Lambda\right)$. Let $Y$ be a symmetric pure jump Hunt process whose jumping intensity kernel $J$ satisfies conditions $(\mathbf{J} 1),(\mathbf{L}),(\mathbf{C}),\left(\mathbf{K}_{\eta}\right)$, and $(\mathbf{S D})$. Then, there exists $c=c\left(\phi, L_{0}, L_{3}, \eta, \Lambda, d\right)$ such that for any $0<r<R_{0} \wedge 1, z \in \partial D$ and any non-negative function $f$ in $\mathbb{R}^{d}$ that is regular harmonic in $D \cap B(z, r)$ with respect to $Y$, and vanishes in $D^{c} \cap B(z, r)$, we have

$$
\frac{f(x)}{f(y)} \leqslant c \sqrt{\frac{\phi\left(\delta_{D}(x)\right)}{\phi\left(\delta_{D}(y)\right)}} \quad \text { for any } x, y \in D \cap B(z, r / 2) \text {. }
$$

The rest of this paper is organized as follows. In Section 2, we solve a martingale-type problem for $Y$ which yields a Dynkin-type formula. Section 3 deals with the isotropic Lévy process $Z$ with Lévy measure $\nu(|x|) d x$. We compute some key upper bounds of the generator of $Z$ on our testing function for $C^{1,1}$ open sets. In Section 4 , we present the key estimates of exit distributions (Theorem 4.2). Section 5 contains the proof of the upper bound of $p_{D}(t, x, y)$. We use Meyer's construction when $|x-y|<c$. Then, by using Lemma 5.1 twice, we prove the upper bound of $p_{D}(t, x, y)$ without using the lower bound of $p(t, x, y)$. In Sections 6 and 7 , we prove the lower bound estimates for $p_{D}(t, x, y)$. First, we consider the case $\delta_{D}(x) \wedge \delta_{D}(y) \geqslant t^{1 / \alpha}$; that is, $x$ and $y$ are kept away from the boundary of $D$. These results are presented in Section 6 and the key estimates of the exit distributions obtained in Section 4 are used in Section 7 to prove the lower bound for all $x, y \in D$. Finally, in Section 8 , as an application of Theorem 4.2, we derive the Green function estimates and the uniform scale-invariant Boundary Harnack inequality with explicit decay rates in $C^{1,1}$ open sets.

Throughout the rest of this paper, positive constants $L_{0}, L_{1}, L_{2}, L_{3}, \gamma_{1}, \gamma_{2}$ can be regarded as fixed. In the statements and the proofs of results, constants $c_{i}=c_{i}(a, b, c, \ldots), i=1,2,3, \ldots$, denote generic constants that depend on $a, b, c, \ldots$, the exact values of which are unimportant. These are given anew in each statement and each proof. The dependence of the constants on the dimension $d \geqslant 1$ is not be mentioned explicitly.

For a function space $\mathbb{H}(U)$ on an open set $U$ in $\mathbb{R}^{d}$, we let $\mathbb{H}_{c}(U):=\{f \in \mathbb{H}(U)$ : $f$ has compact support $\}, \mathbb{H}_{0}(U):=\{f \in \mathbb{H}(U): f$ vanishes at infinity $\}$ and $\mathbb{H}_{b}(U):=\{f \in$ $\mathbb{H}(U): f$ is bounded $\}$. 


\section{Generator of $Y$}

In this section, we assume that $Y$ is the symmetric pure jump Hunt process with the jumping intensity kernel $J$ satisfying the conditions $(\mathbf{J} 1.1),(\mathbf{J 1 . 2})$ and $\left(\mathbf{K}_{\eta}\right)$. Recall that these conditions imply that $Y$ is strong Feller (see [11, Theorem 3.1]).

We define an operator $\mathcal{L}$ by

$$
\mathcal{L} g(x):=P . V . \int(g(x+h)-g(x)) J(x, x+h) d h:=\lim _{\varepsilon \downarrow 0} \mathcal{L}^{\varepsilon} g(x),
$$

where

$$
\mathcal{L}^{\varepsilon} g(x):=\int_{|h|>\varepsilon}(g(x+h)-g(x)) J(x, x+h) d h,
$$

whenever these exist pointwise. Let $g \in C_{c}^{2}\left(\mathbb{R}^{d}\right)$ and $\varepsilon<r<1$, then by $(\mathbf{J 1 . 1})$, we have

$$
\begin{aligned}
& \mathcal{L}^{\varepsilon} g(x)=\kappa(x, x) \int_{\varepsilon<|h|<r}(g(x+h)-g(x)-h \cdot \nabla g(x)) \nu(|h|) d h \\
& \quad+\int_{\varepsilon<|h|<r}(g(x+h)-g(x))(\kappa(x, x+h)-\kappa(x, x)) \nu(|h|) d h \\
& \quad+\int_{r \leqslant|h| \leqslant 1}(g(x+h)-g(x)) \kappa(x, x+h) \nu(|h|) d h+\int_{1<|h|}(g(x+h)-g(x)) J(x, x+h) d h .
\end{aligned}
$$

Since $\left(\mathbf{K}_{\eta}\right)$ holds, we have that

$$
|(g(x+h)-g(x))(\kappa(x, x+h)-\kappa(x, x))| \leqslant\|\nabla g\|_{\infty}\left(L_{3}+2 L_{0}\right)|h|^{\eta+1}, \quad x, h \in \mathbb{R}^{d} .
$$

Since (WS) and the inequality $\eta>\bar{\alpha} / 2>\bar{\alpha}-1$ imply $\int_{|h|<r}|h|^{2} \nu(|h|) d h \leqslant \int_{|h|<r}|h|^{\eta+1} \nu(|h|) d h<$ $\infty, \mathcal{L} g$ is well defined and $\mathcal{L}^{\varepsilon} g$ converges to $\mathcal{L} g$ locally uniformly on $\mathbb{R}^{d}$. Furthermore, for every $0<r<1$,

$$
\begin{aligned}
& \mathcal{L} g(x)=\kappa(x, x) \int_{|h|<r}(g(x+h)-g(x)-h \cdot \nabla g(x)) \nu(|h|) d h \\
& +\int_{|h|<r}(g(x+h)-g(x))(\kappa(x, x+h)-\kappa(x, x)) \nu(|h|) d h \\
& +\int_{r \leqslant|h| \leqslant 1}(g(x+h)-g(x)) \kappa(x, x+h) \nu(|h|) d h+\int_{1<|h|}(g(x+h)-g(x)) J(x, x+h) d h .
\end{aligned}
$$

Now we are ready to prove the following lemma.

Lemma 2.1. There is $C_{2.1}=C 2.1\left(\phi, \eta, L_{0}, L_{3}\right)>0$ such that for any function $g \in C_{c}^{2}\left(\mathbb{R}^{d}\right)$ and $0<r<1$,

$$
\|\mathcal{L} g\|_{\infty} \leqslant \frac{G 2.1}{\phi(r)}\left(r^{2}\left\|\partial^{2} g\right\|_{\infty}+r^{\eta+1}\|\nabla g\|_{\infty}+\|g\|_{\infty}\right)
$$

Proof. By (2.2), (1.4), $\left(\mathbf{K}_{\eta}\right)$ and (J1.2), we obtain that

$$
\begin{aligned}
|\mathcal{L} g(x)| \leqslant c_{0}( & L_{0}\left\|\partial^{2} g\right\|_{\infty} \int_{0}^{r} \frac{s}{\phi(s)} d s+L_{3}\|\nabla g\|_{\infty} \int_{0}^{r} \frac{s^{\eta}}{\phi(s)} d s \\
& \left.+2 L_{0}\|g\|_{\infty} \int_{r}^{1} \frac{d s}{s \phi(s)}\right)+c_{1}\|g\|_{\infty} .
\end{aligned}
$$


For $s \leqslant r$, since $\phi(r) / \phi(s) \leqslant \bar{C}(r / s)^{\bar{\alpha}}$ by (WS) and $\eta>\bar{\alpha} / 2>\bar{\alpha}-1$, we have

$$
\int_{0}^{r} \frac{s^{\eta}}{\phi(s)} d s \leqslant \frac{\bar{C}}{\phi(r)} \frac{1}{\eta+1-\bar{\alpha}} r^{\eta+1} .
$$

For $r<s$, since $\underline{c}(s / r)^{\underline{\alpha}} \leqslant \phi(s) / \phi(r)$ by (WS), we have

$$
\int_{r}^{\infty} \frac{d s}{s \phi(s)} \leqslant \frac{\underline{c}^{-1}}{\phi(r)} r \underline{\alpha} \int_{r}^{\infty} s^{-1-\bar{\alpha}} d s<(\underline{c} \underline{\alpha})^{-1} \frac{1}{\phi(r)} .
$$

Applying (2.5) and (2.6) to (2.4), we conclude that (2.3) hold.

Lemma 2.2. For any $u \in C_{c}^{2}\left(\mathbb{R}^{d}\right)$ and $x \in \mathbb{R}^{d}$, there exists a $\mathbb{P}_{x}$-martingale $M_{t}^{u}$ with respect to the filtration of $Y$ such that

$$
M_{t}^{u}=u\left(Y_{t}\right)-u\left(Y_{0}\right)-\int_{0}^{t} \mathcal{L} u\left(Y_{s}\right) d s
$$

$\mathbb{P}_{x}$-a.s. In particular, for any stopping time $S$ with $\mathbb{E}_{x} S<\infty$ we have

$$
\mathbb{E}_{x} u\left(Y_{S}\right)-u(x)=\mathbb{E}_{x} \int_{0}^{S} \mathcal{L} u\left(Y_{s}\right) d s .
$$

Proof. Let $(A, D(A))$ be the $L^{2}$-generator of the semigroup $T_{t}$ with respect to $Y$. Due to [40, Proposition 2.5], we have $C_{c}^{2}\left(\mathbb{R}^{d}\right) \subset D(A)$ and $\left.A\right|_{C_{c}^{2}\left(\mathbb{R}^{d}\right)}=\left.\mathcal{L}\right|_{C_{c}^{2}\left(\mathbb{R}^{d}\right)}$. Since $T_{t}$ is strongly continuous (see [27, Theorem 1.3.1 and Lemma 1.3.2]) we have that for any $f \in D(A)$ and $t \geqslant 0$,

$$
\left\|\left(T_{t} f-f\right)-\int_{0}^{t} T_{s} A f d s\right\|_{L^{2}}=0
$$

(see e.g. [24, Proposition 1.5]). Hence for $u \in C_{c}^{2}\left(\mathbb{R}^{d}\right)$,

$$
T_{t} u(x)-u(x)=\int_{0}^{t} T_{s} \mathcal{L} u(x) d s, \quad \text { a.e. } x \in \mathbb{R}^{d},
$$

and $\mathcal{L} u$ is bounded by Lemma 2.1.

Let us denote $g_{t}(x)=\int_{0}^{t} T_{s} \mathcal{L} u(x) d s$. First, we show that $g_{t} \in C_{b}\left(\mathbb{R}^{d}\right), t>0$. Note that $g_{t}(x) \leqslant t\|\mathcal{L} u\|_{\infty}$. Hence, since $T_{\varepsilon}$ is strong Feller for any $\varepsilon>0$, we have $T_{\varepsilon} g_{t-\varepsilon} \in C_{b}\left(\mathbb{R}^{d}\right)$ for all $\varepsilon \in(0, t)$. Moreover,

$$
\left|g_{t}(x)-T_{\varepsilon} g_{t-\varepsilon}(x)\right|=\left|g_{\varepsilon}(x)\right| \leqslant \varepsilon|| \mathcal{L} u \|_{\infty} .
$$

Hence, $g_{t}$ is continuous and (2.8) holds for any $x \in \mathbb{R}^{d}$. This and Markov property imply that

$$
M_{t}^{u}=u\left(Y_{t}\right)-u\left(Y_{0}\right)-\int_{0}^{t} \mathcal{L} u\left(Y_{s}\right) d s
$$

is $\mathbb{P}_{x}$-martingale for any $x \in \mathbb{R}^{d}$. Since $\left|M_{t}^{u}\right| \leqslant 2|| u\left\|_{\infty}+t|| \mathcal{L} u\right\|_{\infty}$, by the optional stopping theorem (2.7) follows.

Lemma 2.3. There exists a constant $C_{2.3}=C_{2.3}\left(\phi, \eta, L_{0}, L_{3}\right)>0$ such that, for any $r \in(0,1]$, $x_{0} \in \mathbb{R}^{d}$, and any stopping time $S$ (with respect to the filtration of $Y$ ), we have

$$
\mathbb{P}_{x}\left(\left|Y_{S}-x_{0}\right| \geqslant r\right) \leqslant C 2.3 \frac{\mathbb{E}_{x}[S]}{\phi(r)}, \quad x \in B\left(x_{0}, r / 2\right) .
$$


Proof. Fix $x_{0} \in \mathbb{R}^{d}$. Since this lemma is clear for $\mathbb{E}_{x}[S]=\infty$, we consider the case that $\mathbb{E}_{x}[S]<\infty$ for $x \in B\left(x_{0}, r / 2\right)$. Define a radial function $g \in C_{c}^{\infty}\left(\mathbb{R}^{d}\right)$ such that $-1 \leqslant g \leqslant 0$, with

$$
g(y):= \begin{cases}-1, & \text { if }|y|<1 / 2 \\ 0, & \text { if }|y| \geqslant 1\end{cases}
$$

Then,

$$
\sum_{i=1}^{d}\left\|\frac{\partial}{\partial y_{i}} g\right\|_{\infty}+\sum_{i, j=1}^{d}\left\|\frac{\partial^{2}}{\partial y_{i} \partial y_{j}} g\right\|_{\infty}=c_{1}<\infty
$$

For any $r \in(0,1]$, define $g_{r}(y)=g\left(\frac{x_{0}-y}{r}\right)$ so that $-1 \leqslant g_{r} \leqslant 0$,

$$
g_{r}(y)= \begin{cases}-1, & \text { if }\left|x_{0}-y\right|<r / 2 \\ 0, & \text { if }\left|x_{0}-y\right| \geqslant r\end{cases}
$$

and

$$
\sum_{i=1}^{d}\left\|\frac{\partial}{\partial y_{i}} g_{r}\right\|_{\infty}<c_{1} r^{-1} \text { and } \sum_{i, j=1}^{d}\left\|\frac{\partial^{2}}{\partial y_{i} \partial y_{j}} g_{r}\right\|_{\infty}<c_{1} r^{-2}
$$

By Lemma 2.1, there exists $c_{2}=c_{2}\left(\underline{\alpha}, \underline{c}, \bar{\alpha}, \bar{C}, \eta, L_{0}, L_{3}\right)>0$ such that for $0<r<1$,

$$
\left\|\mathcal{L} g_{r}\right\|_{\infty} \leqslant \frac{c_{2}}{\phi(r)}
$$

Combining Lemma 2.2. (2.9) and (2.11), we find that for any $x \in B\left(x_{0}, r / 2\right)$ with $\mathbb{E}_{x} S<\infty$, we have

$$
\begin{aligned}
& \mathbb{P}_{x}\left(\left|Y_{S}-x_{0}\right| \geqslant r\right)=\mathbb{E}_{x}\left[1+g_{r}\left(Y_{S}\right) ;\left|Y_{S}-x_{0}\right| \geqslant r\right] \\
& \leqslant \mathbb{E}_{x}\left[1+g_{r}\left(Y_{S}\right)\right]=-g_{r}(x)+\mathbb{E}_{x}\left[g_{r}\left(Y_{S}\right)\right]=\mathbb{E}_{x}\left[\int_{0}^{S} \mathcal{L} g_{r}\left(Y_{t}\right) d t\right] \leqslant\left\|\mathcal{L} g_{r}\right\|_{\infty} \mathbb{E}_{x}[S] \\
& \leqslant c_{2} \frac{\mathbb{E}_{x}[S]}{\phi(r)}
\end{aligned}
$$

Recall that for any open set $D \subset \mathbb{R}^{d}, \tau_{D}=\inf \left\{t>0: Y_{t} \notin D\right\}$ denote the first exit time of $D$ by the process $Y_{t}$.

Corollary 2.4. There exists a constant $C_{2.4}=C_{2.4}\left(\phi, \eta, L_{0}, L_{3}\right)>0$ such that, for any $r \in(0,1], x_{0} \in \mathbb{R}^{d}$, and any open sets $U$ and $D$ with $D \cap B\left(x_{0}, r\right) \subset U \subset D$, we have

$$
\mathbb{P}_{x}\left(Y_{\tau_{U}} \in D\right) \leqslant C 2.4 \frac{\mathbb{E}_{x}\left[\tau_{U}\right]}{\phi(r)}, \quad x \in D \cap B\left(x_{0}, r / 2\right)
$$

Proof. Since $D \backslash U \subset B\left(x_{0}, r\right)^{c}$, by Lemma 2.3 we have that for $x \in D \cap B\left(x_{0}, r / 2\right)$

$$
\mathbb{P}_{x}\left(Y_{\tau_{U}} \in D\right) \leqslant \mathbb{P}_{x}\left(\left|Y_{\tau_{U}}-x_{0}\right| \geqslant r\right) \leqslant C_{2.3} \frac{\mathbb{E}_{x}\left[\tau_{U}\right]}{\phi(r)} .
$$




\section{Analysis on $Z$}

Recall that $Z$ is a pure jump isotropic unimodal Lévy process with Lévy measure $\nu(|x|) d x$. Moreover, we assume (SD) holds in this section. The Lévy-Khintchine (characteristic) exponent of $Z$ has the form

$$
\psi(|\xi|)=\int_{\mathbb{R}^{d}}(1-\cos (\xi \cdot x)) \nu(|x|) d x, \quad \xi \in \mathbb{R}^{d} .
$$

Let $Z^{d}$ be the last coordinate of $Z$ and $M_{t}=\sup _{s \leqslant t} Z_{s}^{d}$ and let $L_{t}$ be the local time at 0 for $M_{t}-Z_{t}^{d}$, the last coordinate of $Z$ reflected at the supremum. We consider its right-continuous inverse, $L_{s}^{-1}$ which is called the ascending ladder time process for $X_{t}^{1}$. Define the ascending ladder-height process as $H_{s}=Z_{L_{s}^{-1}}^{d}=M_{L_{s}^{-1}}$. The Laplace exponent of $H_{s}$ is

$$
\kappa(\xi)=\exp \left\{\frac{1}{\pi} \int_{0}^{\infty} \frac{\log \psi(\theta \xi)}{1+\theta^{2}} d \theta\right\}, \quad \xi \geqslant 0 .
$$

(See [25, Corollary 9.7].) The renewal function $V$ of the ascending ladder-height process $H$ is defined as

$$
V(x)=\int_{0}^{\infty} \mathbb{P}\left(H_{s} \leqslant x\right) d s, \quad x \in \mathbb{R} .
$$

then $V(x)=0$ if $x<0$ and $V$ is non-decreasing. Also $V$ is subadditive (see [2, p.74]), that is,

$$
V(x+y) \leqslant V(x)+V(y), \quad x, y \in \mathbb{R},
$$

and $V(\infty)=\infty$. Since the distribution of $Z_{t}^{d}$ is absolutely continuous for every $t>0$ the resolvent measures of $Z_{t}^{d}$ as well, (see [26. Theorem 6]), it follows by [42, Theorem 2] that $V(x)$ is absolutely continuous and harmonic on $(0, \infty)$ for the process $Z_{t}^{d}$. Also, $V^{\prime}$ is a positive harmonic function for $Z_{t}^{d}$ on $(0, \infty)$, hence $V$ is actually (strictly) increasing.

For $r>0$, define Pruitt's function $h(r)=\int_{\mathbb{R}^{d}}\left(1 \wedge|z|^{2} r^{-2}\right) \nu(d z)$ (e.g., see [38]). By [4, Corollary 3] and [6, Proposition 2.4] (see also [2, p.74]), we first note that

$$
h(r) \asymp[V(r)]^{-2} \asymp \kappa\left(r^{-1}\right) \asymp \psi\left(r^{-1}\right) \quad \text { for any } r>0 .
$$

Clearly, (WS) implies that $s \rightarrow \phi\left(s^{-1}\right)^{-1}$ also satisfies (WS), that is, using the notation in [4], $s \rightarrow \phi\left(s^{-1}\right)^{-1} \in \operatorname{WLSC}(\underline{\alpha}, \underline{c}, 0) \cap \operatorname{WUSC}(\bar{\alpha}, \bar{C}, 0)$. So by (1.2) and [4, Proposition 28], we have that

$$
\psi(r) \asymp \phi\left(r^{-1}\right)^{-1} \quad \text { for any } r>0 .
$$

Combining these observations, we conclude that

$$
V(r) \asymp[\phi(r)]^{1 / 2} \quad \text { and } \quad \nu(r) \asymp[V(r)]^{-2} r^{-d} \quad \text { for any } r>0 .
$$

So by (WS), there exists $C_{V}:=(\underline{c}, \bar{C}, d)>1$ such that

$$
C_{V}^{-1}\left(\frac{R}{r}\right)^{\underline{\alpha} / 2} \leqslant \frac{V(R)}{V(r)} \leqslant C_{V}\left(\frac{R}{r}\right)^{\bar{\alpha} / 2} \quad \text { for any } 0<r \leqslant R .
$$

Define $w(x):=V\left(\left(x_{d}\right)^{+}\right)$and $\mathbb{H}:=\left\{x=\left(\widetilde{x}, x_{d}\right) \in \mathbb{R}^{d}: x_{d}>0\right\}$. Since the renewal function $V$ is harmonic on $(0, \infty)$ for $Z^{d}$, by the strong Markov Property $w$ is harmonic in $\mathbb{H}$ with respect to $Z$. 
Proposition 3.1. $x \rightarrow V(x)$ is twice-differentiable for any $x>0$, and there exists $93.1>0$ such that

$$
\left|V^{\prime \prime}(x)\right| \leqslant C 3.1 \frac{V^{\prime}(x)}{x \wedge 1} \quad \text { and } \quad V^{\prime}(x) \leqslant 93.1 \frac{V(x)}{x \wedge 1}, \quad x>0 .
$$

Proof. Let $f\left(\left(\widetilde{y}, x_{d}\right)\right)=V^{\prime}\left(\left(x_{d}\right)^{+}\right)$for $\widetilde{y} \in \mathbb{R}^{d-1}$. Then $f$ is harmonic in $\mathbb{H}$. The assumption [36, (A)] is satisfied by (SD). Hence, by Theorem 1.1 therein, we get for any $x>0$

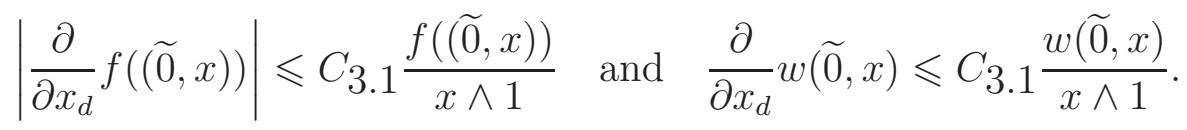

These imply the claim of proposition, because $V(x)=w(\widetilde{0}, x)$ and $V^{\prime}(x)=f(\widetilde{0}, x), x>0$.

Proposition 3.2. For $\lambda>0$, there exists $C_{3.2}=C_{3.2}(d, \lambda)>0$ such that for any $r>0$, we have

$$
\sup _{\left\{x \in \mathbb{R}^{d}: 0<x_{d} \leqslant \lambda r\right\}} \int_{B(x, r)^{c}} w(y) \nu(|x-y|) d y<\frac{93.2}{V(r)} .
$$

Proof. Since $w(x+z)=V\left(x_{d}+z_{d}\right) \leqslant V\left(x_{d}\right)+V(|z|)$ for $x_{d}>0$, it follows that

$$
\begin{aligned}
\int_{B(x, r)^{c}} w(y) \nu(|x-y|) d y & =\int_{B(0, r)^{c}} w(x+z) \nu(|z|) d z \\
& \leqslant V\left(x_{d}\right) \int_{B(0, r)^{c}} \nu(|z|) d z+\int_{B(0, r)^{c}} V(|z|) \nu(|z|) d z .
\end{aligned}
$$

By [6, (2.23) and Lemma 3.5], we have that

$$
\sup _{\left\{x \in \mathbb{R}^{d}: 0<x_{d} \leqslant \lambda r\right\}} \int_{B(x, r)^{c}} w(y) \nu(|x-y|) d y \leqslant c_{1}\left(\frac{V(\lambda r)}{[V(r)]^{2}}+\frac{1}{V(r)}\right) .
$$

Since $V$ is subadditive, $V(\lambda r) \leqslant(\lambda+1) V(r)$ for any $\lambda>0$, and therefore we conclude the result.

For any function $f: \mathbb{R}^{d} \rightarrow \mathbb{R}$ and $x \in \mathbb{R}^{d}$, we define an operator as follows:

$$
\begin{aligned}
\mathcal{L}_{Z} f(x) & :=P . V . \int_{\mathbb{R}^{d}}(f(y)-f(x)) \nu(|x-y|) d y:=\lim _{\varepsilon \downarrow 0} \mathcal{L}_{Z}^{\varepsilon} f(x), \\
\mathcal{D}\left(\mathcal{L}_{Z}\right) & :=\left\{f \in C^{2}\left(\mathbb{R}^{d}\right): P . V . \int_{\mathbb{R}^{d}}(f(y)-f(x)) \nu(|x-y|) d y \text { exists and is finite. }\right\},
\end{aligned}
$$

where

$$
\mathcal{L}_{Z}^{\varepsilon} f(x):=\int_{B(x, \varepsilon)^{c}}(f(y)-f(x)) \nu(|x-y|) d y .
$$

Recall that $C_{0}^{2}\left(\mathbb{R}^{d}\right)$ be the collection of $C^{2}$ functions in $\mathbb{R}^{d}$ vanishing at infinity. It is well known that $C_{0}^{2}\left(\mathbb{R}^{d}\right) \subset \mathcal{D}\left(\mathcal{L}_{Z}\right)$ and that, by the rotational symmetry of $Z,\left.A_{Z}\right|_{C_{0}^{2}\left(\mathbb{R}^{d}\right)}=\left.\mathcal{L}_{Z}\right|_{C_{0}^{2}\left(\mathbb{R}^{d}\right)}$ where $A_{Z}$ is the infinitesimal generator of $Z$ (e.g. [39, Theorem 31.5]). Hence, we see that Dynkin formula holds for $\mathcal{L}_{Z}$ : for each $g \in C_{0}^{2}\left(\mathbb{R}^{d}\right)$ and any bounded open subset $U$ of $\mathbb{R}^{d}$ we have

$$
\mathbb{E}_{x} \int_{0}^{\tau_{U}} \mathcal{L}_{Z} g\left(Z_{t}\right) d t=\mathbb{E}_{x}\left[g\left(Z_{\tau_{U}}\right)\right]-g(x)
$$


Theorem 3.3. For any $x \in \mathbb{H}, \mathcal{L}_{Z} w(x)$ is well-defined and $\mathcal{L}_{Z} w(x)=0$.

Proof. By subadditivity of $V,|w(y)-w(x)| \leqslant V\left(\left|y_{d}-x_{d}\right|\right) \leqslant V(|x-y|)$ for $x \in \mathbb{H}$. By $[\underline{6}$, Lemma 3.5], it follows that for any $\varepsilon \in(0,1 / 2)$

$$
\left|\int_{B(x, \varepsilon)^{c}}(w(y)-w(x)) \nu(|x-y|) d y\right| \leqslant \int_{B(0, \varepsilon)^{c}} V(|z|) \nu(|z|) d z<\frac{c_{1}}{V(\varepsilon)}<\infty .
$$

Thus $\mathcal{L}_{Z}^{\varepsilon} w(x)$ is well defined in $\mathbb{H}$ and

$$
\mathcal{L}_{Z}^{\varepsilon} w(x)=\int_{B(x, \varepsilon)^{c}}\left(w(y)-w(x)-\mathbf{1}_{\{|x-y|<1\}}(x-y) \cdot \nabla w(x)\right) \nu(|x-y|) d y .
$$

Since Proposition 3.1 implies $V^{\prime \prime}(s)$ exists and so $w$ is twice differentiable in $\mathbb{H}$, we have that

$$
x \mapsto \int_{B(x, \varepsilon)}(w(y)-w(x)-(x-y) \cdot \nabla w(x)) \nu(|x-y|) d y
$$

converges to 0 locally uniformly in $\mathbb{H}$ as $\varepsilon \downarrow 0$. From (3.8), we see that $\mathcal{L}_{Z}^{\varepsilon} w(x)$ converges to

$$
\mathcal{L}_{Z} w(x)=\int_{\mathbb{R}^{d}}\left(w(y)-w(x)-\mathbf{1}_{\{|x-y|<1\}}(x-y) \cdot \nabla w(x)\right) \nu(|x-y|) d y
$$

locally uniformly in $\mathbb{H}$ as $\varepsilon \downarrow 0$.

For every $x \in \mathbb{H}, z \in B\left(x,\left(\varepsilon \wedge x_{d}\right) / 2\right)$ and $y \in B(z, \varepsilon)^{c}$, it holds that $|y-z| / 2 \leqslant|x-y| \leqslant$ $3|y-z| / 2$. Since $r \rightarrow \nu(r)$ is decreasing, using Proposition 3.1

$$
\begin{aligned}
& \mathbf{1}_{\{|y-z|>\varepsilon\}}\left|w(y)-w(z)-\mathbf{1}_{\{|y-z|<1\}}(y-z) \cdot \nabla w(z)\right| \nu(|y-z|) \\
& \leqslant c_{2}\left(\sup _{\varepsilon / 2<s<x_{d}+2} V^{\prime \prime}(s)\right)|x-y|^{2} \mathbf{1}_{\{\varepsilon / 2<|x-y|<2\}} \nu(|x-y| / 2) \\
& \quad+\left(w(y)+V\left(x_{d}+1\right)\right) \mathbf{1}_{\{|x-y|>1 / 2\}} \nu(|x-y| / 2) .
\end{aligned}
$$

So applying the dominated convergence theorem with Proposition 3.2 and the fact that $\nu$ is a Lévy density, we obtain that $x \rightarrow \mathcal{L}_{Z}^{\varepsilon} w(x)$ is continuous for each $\varepsilon$. Therefore, the function $\mathcal{L}_{Z} w(x)$ is continuous in $\mathbb{H}$.

Let $U_{1}$ and $U_{2}$ be relatively compact open subsets of $\mathbb{H}$ satisfying $\overline{U_{1}} \subset U_{2} \subset \overline{U_{2}} \subset \mathbb{H}$ and $0<r_{0}:=\operatorname{dist}\left(U_{1}, U_{2}^{c}\right)<1$. By Proposition 3.2 ,

$$
\begin{aligned}
\int_{U_{1}} \int_{U_{2}^{c}} w(y) \nu(|x-y|) d y d x & \leqslant\left|U_{1}\right| \sup _{x \in U_{1}} \int_{U_{2}^{c}} w(y) \nu(|x-y|) d y \\
& \leqslant\left|U_{1}\right| \sup _{x \in U_{1}} \int_{B\left(x, r_{0}\right)^{c}} w(y) \nu(|x-y|) d y<\infty .
\end{aligned}
$$

Since $w$ is harmonic, $w\left(Z_{\tau_{U_{1}}}\right) \in L^{1}\left(\mathbb{P}_{x}\right)$ and

$$
\sup _{x \in U_{1}} \mathbb{E}_{x}\left[\mathbf{1}_{U_{2}^{c}}\left(Z_{\tau_{U_{1}}}\right) w\left(Z_{\tau_{U_{1}}}\right)\right] \leqslant \sup _{x \in U_{1}} \mathbb{E}_{x}\left[w\left(Z_{\tau_{U_{1}}}\right)\right]=\sup _{x \in U_{1}} w(x)<\infty .
$$

From (3.9) and (3.10), the conditions [9, (2.4), (2.6)] hold and by [9, Lemma 2.3, Theorem 2.11(ii)], we have that for any $f \in C_{c}^{2}(\mathbb{H})$,

$$
0=\int_{\mathbb{R}^{d}} \int_{\mathbb{R}^{d}}(w(y)-w(x))(f(y)-f(x)) \nu(|x-y|) d x d y .
$$


For $f \in C_{c}^{2}(\mathbb{H})$ with $\operatorname{supp}(f) \subset \overline{U_{1}} \subset U_{2} \subset \overline{U_{2}} \subset \mathbb{H}$,

$$
\begin{aligned}
& \int_{\mathbb{R}^{d}} \int_{\mathbb{R}^{d}}|w(y)-w(x)||f(y)-f(x)| \nu(|x-y|) d x d y \\
= & \int_{U_{1}} \int_{U_{2}}|w(y)-w(x)||f(y)-f(x)| \nu(|x-y|) d x d y+2 \int_{U_{1}} \int_{U_{2}^{c}}|w(y)-w(x)||f(y)| \nu(|x-y|) d x d y \\
\leqslant & c_{2} \int_{U_{1} \times U_{2}}|x-y|^{2} \nu(|x-y|) d x d y+2|| f \|_{\infty} \int_{U_{1}} \int_{U_{2}^{c}} w(y) \nu(|x-y|) d x d y \\
& +2|| f \|_{\infty}\left|U_{1}\right|\left(\sup _{y \in U_{1}} w(y)\right) \int_{U_{1}} \int_{U_{2}^{c}} \nu(|x-y|) d x d y
\end{aligned}
$$

and it is finite from (3.9) and the fact that $\nu$ is a Lévy density. Applying the dominated convergence theorem with (3.11) and (3.12), for any $f \in C_{c}^{2}(\mathbb{H})$, we have

$$
\begin{aligned}
0 & =\lim _{\varepsilon \downarrow 0} \int_{\left\{(x, y) \in \mathbb{R}^{d} \times \mathbb{R}^{d}:|x-y|>\varepsilon\right\}}(w(y)-w(x))(f(y)-f(x)) \nu(|x-y|) d x d y \\
& =-2 \lim _{\varepsilon \downarrow 0} \int_{\mathbb{H}} f(x)\left(\int_{\left\{y \in \mathbb{R}^{d}:|x-y|>\varepsilon\right\}}(w(y)-w(x)) \nu(|x-y|) d y\right) d x=-2 \int_{\mathbb{H}} f(x) \mathcal{L}_{Z} w(x) d x .
\end{aligned}
$$

We have used Fubini's theorem and the fact that $\mathcal{L}_{Z}^{\varepsilon} w \rightarrow \mathcal{L}_{Z} w$ converges uniformly on the support $f$. Hence, by the continuity of $\mathcal{L}_{Z} w$, we have $\mathcal{L}_{Z} w(x)=0$ in $\mathbb{H}$.

Proposition 3.4. Suppose that $D$ is a $C^{1,1}$ open set in $\mathbb{R}^{d}$ with characteristics $\left(R_{0}, \Lambda\right)$. For any $z \in \partial D$ and $r \leqslant 1 \wedge R_{0}$, we define

$$
h_{r}(y)=h_{r, z}(y):=V\left(\delta_{D}(y)\right) \mathbf{1}_{D \cap B(z, r)}(y) .
$$

There exists $C_{3.4}=C$ 3.4 $(\phi, \Lambda, d)>0$ independent of $z$ such that $\mathcal{L}_{Z} h$ is well-defined in $D \cap$ $B(z, r / 4)$ and

$$
\left|\mathcal{L}_{Z} h_{r}(x)\right| \leqslant \frac{9 \overline{3.4}}{V(r)} \quad \text { for all } x \in D \cap B(z, r / 4)
$$

Proof. Since the case of $d=1$ is easier, we give the proof only for $d \geqslant 2$. Without loss of generality, we assume that $R_{0}<1$ and $\Lambda>1$. For $x \in D \cap B(z, r / 4)$, let $z_{x} \in \partial D$ be the point satisfying $\delta_{D}(x)=\left|x-z_{x}\right|$. Let $\varphi$ be a $C^{1,1}$ function and $C S=C S_{z_{x}}$ be an orthonormal coordinate system with $z_{x}$ chosen so that $\varphi(\widetilde{0})=0, \nabla \varphi(\widetilde{0})=(0, \ldots, 0),\|\nabla \varphi\|_{\infty} \leqslant$ $\Lambda,|\nabla \varphi(\widetilde{y})-\nabla \varphi(\widetilde{z})| \leqslant \Lambda|\widetilde{y}-\widetilde{z}|$, and $x=\left(\widetilde{0}, x_{d}\right), D \cap B\left(z_{x}, R_{0}\right)=\left\{y=\left(\widetilde{y}, y_{d}\right) \in B\left(0, R_{0}\right)\right.$ in $C S:$ $\left.y_{d}>\varphi(\widetilde{y})\right\}$. We fix the function $\varphi$ and the coordinate system $C S$, and we define a function $g_{x}(y)=V\left(\delta_{\mathbb{H}}(y)\right)=V\left(y_{d}\right)$, where $\mathbb{H}=\left\{y=\left(\widetilde{y}, y_{d}\right)\right.$ in $\left.C S: y_{d}>0\right\}$ is the half space in $C S$.

Note that $h_{r}(x)=g_{x}(x)$, and that $\mathcal{L}_{Z}\left(h_{r}-g_{x}\right)=\mathcal{L}_{Z} h_{r}$ by Theorem 3.3. So, it suffices to show that $\mathcal{L}_{Z}\left(h_{r}-g_{x}\right)$ is well defined and that there exists a constant $c_{1}=c_{1}(\underline{\alpha}, \underline{c}, \bar{\alpha}, \bar{C}, \Lambda, d)>0$ independent of $x \in D \cap B(z, r / 4)$ and $z \in \partial D$ such that

$$
\int_{D \cup \mathbb{H}}\left|h_{r}(y)-g_{x}(y)\right| \nu(|x-y|) d y \leqslant c_{1} V(r)^{-1} .
$$

We define $\widehat{\varphi}: B(\widetilde{0}, r) \rightarrow \mathbb{R}$ by $\widehat{\varphi}(\widetilde{y}):=2 \Lambda|\widetilde{y}|^{2}$. Since $\nabla \varphi(\widetilde{0})=0$, by the mean value theorem we have $-\widehat{\varphi}(\widetilde{y}) \leqslant \varphi(\widetilde{y}) \leqslant \widehat{\varphi}(\widetilde{y})$ for any $y \in D \cap B(x, r / 2)$ and so that

$$
\begin{aligned}
\left\{z=\left(\widetilde{z}, z_{d}\right) \in B(x, r / 2): z_{d} \geqslant\right. & \widehat{\varphi}(\widetilde{z})\} \subset D \cap B(x, r / 2) \\
& \subset\left\{z=\left(\widetilde{z}, z_{d}\right) \in B(x, r / 2): z_{d} \geqslant-\widehat{\varphi}(\widetilde{z})\right\} .
\end{aligned}
$$


Let $A:=\left\{y \in(D \cup \mathbb{H}) \cap B(x, r / 4):-\widehat{\varphi}(\widetilde{y}) \leqslant y_{d} \leqslant \widehat{\varphi}(\widetilde{y})\right\}$ and $E:=\left\{y \in B(x, r / 4): y_{d}>\right.$ $\widehat{\varphi}(\widetilde{y})\} \subset D$. We will prove (3.14) by showing that I + II + III $\leqslant c_{1} V(r)^{-1}$, where

$$
\begin{aligned}
& \mathrm{I}:=\int_{B(x, r / 4)^{c}}\left(h_{r}(y)+g_{x}(y)\right) \nu(|x-y|) d y \\
& \mathrm{II}:=\int_{A}\left(h_{r}(y)+g_{x}(y)\right) \nu(|x-y|) d y, \quad \text { and } \quad \text { III }:=\int_{E}\left|h_{r}(y)-g_{x}(y)\right| \nu(|x-y|) d y .
\end{aligned}
$$

First, since $h_{r} \leqslant V(r)$, by [6, (2.23)] and Proposition [3.2, we have

$$
\begin{aligned}
\mathrm{I} & \leqslant V(r) \int_{B(x, r / 4)^{c}} \nu(|x-y|) d y+\sup _{\left\{z \in \mathbb{R}^{d}: 0<z_{d}<r\right\}} \int_{B(z, r / 4)^{c} \cap \mathbb{H}} g_{x}(y) \nu(|z-y|) d y \\
& \leqslant c_{2} V(r)^{-1}+\sup _{\left\{z \in \mathbb{R}^{\left.d: 0<z_{d}<r\right\}}\right.} \int_{B(z, r / 4)^{c} \cap \mathbb{H}} w(y) \nu(|z-y|) d y \leqslant\left(c_{2}+C_{(3.2}\right)^{V(r)^{-1} .}
\end{aligned}
$$

For $y \in A$, since $\widehat{\varphi}(\widetilde{y}) \leqslant 2 \Lambda|\widetilde{y}|$ and $V$ is increasing and subadditive, we observe that $h_{r}(y)+g_{x}(y) \leqslant 2 V(2 \widehat{\varphi}(\widetilde{y})) \leqslant c_{3} V(|\widetilde{y}|)$. For $s \leqslant r / 4$, note that $m_{d-1}\left(\left\{y:|\widetilde{y}|=s,-\widehat{\varphi}(\widetilde{y}) \leqslant y_{d} \leqslant\right.\right.$ $\widehat{\varphi}(\widetilde{y})\}) \leqslant c_{4} s^{d}$ where $m_{d-1}(d y)$ is the surface measure on $\mathbb{R}^{d-1}$. Thus $\int_{|\widetilde{y}|=s} \mathbf{1}_{A}(y) V(|\widetilde{y}|) \nu(|\widetilde{y}|)$ $m_{d-1}(d y) \leqslant c_{4} V(s) \nu(s) s^{d}$ for $0<s<r / 4$. From (3.4), we note that $V(s)^{-1} \leqslant C_{V} V(r)^{-1}(r / s)^{\bar{\alpha} / 2}$ for $s \leqslant r$. Hence, by (3.3) and (1.2),

$$
\begin{aligned}
\mathrm{II} & \leqslant c_{3} c_{4} \int_{0}^{r} V(s) \nu(s) s^{d} d s \\
& \leqslant c_{5} \int_{0}^{r} V(s)^{-1} s d s \leqslant c_{4} \cdot C_{V} V(r)^{-1} r^{\bar{\alpha} / 2} \int_{0}^{r} s^{-\bar{\alpha} / 2} d s \\
& =c_{5} \cdot C_{V} V(r)^{-1} \frac{1}{1-\bar{\alpha} / 2} r^{1} \leqslant c_{6} V(r)^{-1}
\end{aligned}
$$

for some positive constant $c_{6}=c_{6}(\bar{\alpha}, \underline{c}, \bar{C}, \Lambda, d)$.

When $y \in E$, we have that $\left|y_{d}-\delta_{D}(y)\right| \leqslant \Lambda|\widetilde{y}|^{2}$. Indeed, if $0<y_{d}=\delta_{\mathbb{H}}(y) \leqslant \delta_{D}(y)$ and $y \in E, \delta_{D}(y) \leqslant y_{d}+|\varphi(\widetilde{y})| \leqslant y_{d}+\Lambda|\widetilde{y}|^{2}$. Since we assume that $R_{0}<1$ and $\Lambda>1$, if $y_{d}=\delta_{\mathbb{H}}(y) \geqslant \delta_{D}(y)$ and $y \in E$, the interior ball condition implies that

$$
\begin{aligned}
y_{d}-\delta_{D}(y) & \leqslant y_{d}-R_{0}+\sqrt{|\widetilde{y}|^{2}+\left(R_{0}-y_{d}\right)^{2}}=\frac{|\widetilde{y}|^{2}}{\sqrt{|\widetilde{y}|^{2}+\left(R_{0}-y_{d}\right)^{2}}+\left(R_{0}-y_{d}\right)} \\
& \leqslant \frac{|\widetilde{y}|^{2}}{2\left(R_{0}-y_{d}\right)} \leqslant \frac{|\widetilde{y}|^{2}}{R_{0}} \leqslant \Lambda|\widetilde{y}|^{2} .
\end{aligned}
$$

Furthermore, $\left|y_{d}-\delta_{D}(y)\right| \leqslant \Lambda|\widetilde{y}|^{2}$ yields that

$$
\Lambda|\widetilde{y}|^{2} \leqslant y_{d}-\Lambda|\widetilde{y}|^{2} \leqslant y_{d} \wedge \delta_{D}(y) \quad \text { and } \quad y_{d} \vee \delta_{D}(y)-\left(y_{d}-\Lambda|\widetilde{y}|^{2}\right) \leqslant 2 \Lambda|\widetilde{y}|^{2} .
$$

Hence, by the mean value Theorem and the scale invariant Harnack inequality for $Z^{d}$ ([11, Theorem 1.4]) applying to $V^{\prime}$, we have that

$$
\begin{aligned}
\left|h_{r}(y)-g_{x}(y)\right| & =\left|V\left(\delta_{D}(y)\right)-V\left(y_{d}\right)\right| \leqslant \sup _{u \in\left[\left(y_{d} \wedge \delta_{D}(y), y_{d} \vee \delta_{D}(y)\right]\right.} V^{\prime}(u)\left|\delta_{D}(y)-y_{d}\right| \\
& \leqslant \sup _{u \in\left[y_{d}-\Lambda|\widetilde{y}|^{2}, y_{d} \vee \delta_{D}(y)\right]} V^{\prime}(u)\left|\delta_{D}(y)-y_{d}\right| \\
& \leqslant c_{7} \inf _{u \in\left[y_{d}-\Lambda|\widetilde{y}|^{2}, y_{d} \vee \delta_{D}(y)\right]} V^{\prime}(u)|\widetilde{y}|^{2} \leqslant 2 \Lambda c_{7} V^{\prime}\left(y_{d}-\frac{1}{2} \widehat{\varphi}(\widetilde{y})\right)|\widetilde{y}|^{2} .
\end{aligned}
$$


Since $E \subset\left\{\left(\widetilde{y}, y_{d}\right):|\widetilde{y}|<r / 4, \widehat{\varphi}(\widetilde{y})<y_{d}<\frac{1}{2} \widehat{\varphi}(\widetilde{y})+r / 2\right\}$, using with (3.15) and the polar coordinates for $|\widetilde{y}|=v$, we first see that

$$
\begin{aligned}
\mathrm{III} & \leqslant 2 \Lambda c_{7} \int_{E} V^{\prime}\left(y_{d}-\frac{1}{2} \widehat{\varphi}(\widetilde{y})\right)|\widetilde{y}|^{2} \nu(|x-y|) d y \\
& \leqslant c_{8} \int_{0}^{r / 4} \int_{\widehat{\varphi}(v)}^{\frac{1}{2} \widehat{\varphi}(v)+r / 2} V^{\prime}\left(y_{d}-\frac{1}{2} \widehat{\varphi}(v)\right) \nu\left(\left(v^{2}+\left|y_{d}-x_{d}\right|^{2}\right)^{1 / 2}\right) v^{d} d y_{d} d v
\end{aligned}
$$

Let $s:=y_{d}-\frac{1}{2} \widehat{\varphi}(v)$. Since $\left(v^{2}+\left|y_{d}-x_{d}\right|^{2}\right)^{1 / 2} \geqslant\left(v+\left|y_{d}-x_{d}\right|\right) / 2$ and $\nu$ is decreasing, by (1.2) and (3.3), we have that

$$
\begin{aligned}
\nu\left(\left(v^{2}+\left|y_{d}-x_{d}\right|^{2}\right)^{1 / 2}\right) v^{d} & \leqslant \nu\left(\left(v+\left|s+\frac{1}{2} \widehat{\varphi}(r)-x_{d}\right|\right) / 2\right)\left(v+\left(\left|s+\frac{1}{2} \widehat{\varphi}(v)-x_{d}\right|\right)^{d}\right. \\
& \leqslant c_{9} V\left(v+\left|s+\frac{1}{2} \widehat{\varphi}(v)-x_{d}\right|\right)^{-2}
\end{aligned}
$$

For $g(s):=\sup _{u \geqslant s}(V(u) / u), V^{\prime}(s) \leqslant C_{3.1}(V(s) / s) \leqslant C_{3.1} g(s)$ by Proposition 3.1. Therefore we have that

$$
\begin{aligned}
\mathrm{III} & \leqslant c_{8} \cdot c_{9} \int_{0}^{r / 2} \int_{0}^{r / 2} V^{\prime}(s) V\left(v+\left|s+\frac{1}{2} \widehat{\varphi}(v)-x_{d}\right|\right)^{-2} d s d v \\
& \leqslant c_{10} \int_{0}^{r / 2} \int_{0}^{r / 2} g(s) V\left(v+\left|s+\frac{1}{2} \widehat{\varphi}(v)-x_{d}\right|\right)^{-2} d s d v .
\end{aligned}
$$

Applying [34, Lemma 4.4] with non-increasing functions $g(s)$ and $f(s):=V(s)^{-2}$ and $x(r)=$ $s+\frac{1}{2} \widehat{\varphi}(r)$, we have that

$$
\mathrm{III} \leqslant c_{11} \int_{0}^{3 r / 4} G(u) V(u)^{-2} d u .
$$

where $G(u)=\int_{0}^{u} g(s) d s$. By subadditivity of $V$ and (3.4), $G(u) \leqslant 2 \int_{0}^{u}(V(s) / s) d s \leqslant c_{12} V(u)$. Using (3.4) again, we conclude that

$$
\mathrm{III} \leqslant c_{11} \cdot c_{12} \int_{0}^{r} V(u)^{-1} d u \leqslant c_{13} V(r)^{-1} r^{\bar{\alpha} / 2} \int_{0}^{r} u^{-\bar{\alpha} / 2} d u \leqslant c_{14} V(r)^{-1}
$$

for some positive constant $c_{14}:=c_{14}(\underline{\alpha}, \bar{\alpha}, \underline{c}, \bar{C}, d)$.

\section{Estimates on exit distributions for $Y$}

In this section we give some key estimates on exit distributions for $Y$. Throughout this section, we assume that $Y$ is the symmetric pure jump Hunt process with the jumping intensity kernel $J$ satisfying the conditions $(\mathbf{J} 1.1),(\mathbf{J} 1.2),(\mathrm{SD})$ and $\left(\mathbf{K}_{\eta}\right)$.

For any $x \in \mathbb{R}^{d}$, stopping time $S$ (with respect to the filtration of $Y$ ), and non-negative measurable function $f$ on $\mathbb{R}_{+} \times \mathbb{R}^{d} \times \mathbb{R}^{d}$ with $f(s, y, y)=0$ for all $y \in \mathbb{R}^{d}$ and $s \geqslant 0$, we have the following Lévy system:

$$
\mathbb{E}_{x}\left[\sum_{s \leqslant S} f\left(s, Y_{s-}, Y_{s}\right)\right]=\mathbb{E}_{x}\left[\int_{0}^{S}\left(\int_{\mathbb{R}^{d}} f\left(s, Y_{s}, y\right) J\left(Y_{s}, y\right) d y\right) d s\right]
$$

(e.g., see [19, Appendix A]).

Throughout this section, we assume that $D$ is a $C^{1,1}$ open set with $C^{1,1}$ characteristics $\left(R_{0}, \Lambda\right)$, and without loss of generality, we will assume that $R_{0}<1$ and $\Lambda>1$. Recall that the function $h_{r}(y)=h_{r, z}(y)$ is defined in Proposition 3.4 . 
Lemma 4.1. Let $r \leqslant R_{0} / 2$. For any $z \in \partial D$ and $k \in \mathbb{N}$, let $B_{k}:=\{y \in D \cap B(z, r / 4)$ : $\left.\delta_{D \cap B(z, r / 4)}(y) \geqslant 2^{-k}\right\}$. Then, for every $u \in \mathbb{R}^{d}$ and $k \in \mathbb{N}$ with $|u|<2^{-k}<2^{-8} r$,

$$
\mathcal{L}^{u} h_{r, z}(w):=\lim _{\varepsilon \downarrow 0} \int_{|(w-u)-y|>\varepsilon}\left(h_{r, z}(y)-h_{r, z}(w-u)\right) J(w, u+y) d y
$$

is well defined in $B_{k}$ and there exists $Q_{4.1}=G_{4.1}\left(\phi, L_{0}, L_{3}, \Lambda, \eta, d\right)>0$ independent of $z \in \partial D$, $k \in \mathbb{N}$ with $2^{-k+8}<r \leqslant R_{0}$ such that

$$
\left|\mathcal{L}^{u} h_{r, z}(w)\right| \leqslant \frac{94.1}{V(r)} \quad \text { for all } w \in B_{k},|u|<2^{-k} .
$$

Proof. We fix $z \in \partial D$ and use the short notation $h_{r}(y)=h_{r, z}(y)$. For any $w \in B_{k}$ and $|u|<2^{-k}<2^{-8} r$, let $x:=w-u \in B(z, r / 4)$. Define $\kappa_{u}(x, y):=\kappa(u+x, u+y)$, and for $\varepsilon<2^{-k-1}$ denote $A_{\varepsilon}(x)$ and $L(x)$ by

$$
\begin{aligned}
A_{\varepsilon}(x) & :=\int_{\varepsilon<|x-y| \leqslant 1}\left(h_{r}(y)-h_{r}(x)\right)\left(\kappa_{u}(x, y)-\kappa_{u}(x, x)\right) \nu(|x-y|) d y, \\
L(x) & :=\int_{1<|x-y|}\left(h_{r}(y)-h_{r}(x)\right)\left(J(x, y)-\kappa_{u}(x, x) \nu(|x-y|)\right) d y,
\end{aligned}
$$

so that

$$
\begin{aligned}
& \int_{\varepsilon<|x-y| \leqslant 1}\left(h_{r}(y)-h_{r}(x)\right) \kappa_{u}(x, y) \nu(|x-y|) d y+\int_{1<|x-y|}\left(h_{r}(y)-h_{r}(x)\right) J(x, y) d y \\
= & A_{\varepsilon}(x)+\kappa_{u}(x, x) \cdot \mathcal{L}_{Z}^{\varepsilon} h_{r}(x)+L(x)
\end{aligned}
$$

where $\mathcal{L}_{Z}^{\varepsilon}$ is defined in $(3.6)$.

By the definition of $h_{r}$, (1.4), (1.2) and (J1.2), for $r \leqslant R_{0}<1$, we first obtain that

$$
|L(x)| \leqslant c_{0}\left(\left|\int_{1<|x-y|} J(x, y) d y\right|+\left|\int_{1<|x-y|} \nu(|x-y|) d y\right|\right) \leqslant c_{1} V(r)^{-1} .
$$

On the other hand,

$$
\begin{aligned}
\left|A_{\varepsilon}(x)\right| & \leqslant\left(\int_{|x-y|<r / 2}+\int_{r / 2 \leqslant|x-y|}\right)\left|h_{r}(y)-h_{r}(x)\right|\left|\kappa_{u}(x, y)-\kappa_{u}(x, x)\right| \nu(|x-y|) d y \\
& =: \operatorname{I}(x)+\operatorname{II}(x) .
\end{aligned}
$$

For $|x-y|<r / 2,\left|h_{r}(y)-h_{r}(x)\right| \leqslant V(|x-y|)$ by subadditivity of $V$, and $V(r) / V(|x-y|) \leqslant$ $C_{V}(r /|x-y|)^{\bar{\alpha} / 2}$ by (3.4). Also $\left|\kappa_{u}(x, y)-\kappa_{u}(x, x)\right| \leqslant L_{3}|x-y|^{\eta}$ by the assumption $\left(\mathbf{K}_{\eta}\right)$. Hence (1.2) and (3.3) imply that

$$
\begin{aligned}
|\mathrm{I}(x)| & \leqslant c_{2} \int_{|x-y|<r} V(|x-y|)^{-1}|x-y|^{\eta-d} d y \\
& \leqslant c_{2} C_{V} V(r)^{-1} r^{\bar{\alpha} / 2} \int_{|x-y|<r}|x-y|^{-\bar{\alpha} / 2+\eta-d} d y \leqslant c_{3} V(r)^{-1}
\end{aligned}
$$

for some positive constant $c_{3}:=c_{3}\left(\bar{\alpha}, \underline{c}, \bar{C}, L_{3}, \eta, d\right)$. The last inequality holds since $\eta>\bar{\alpha} / 2$. To obtain the upper bound of $\operatorname{II}(x)$, note that $\left|h_{r}(y)-h_{r}(x)\right| \leqslant 2 V(|x-y|)$ for $r / 2 \leqslant \mid x-$ $y \mid$. Indeed, if $y \in(D \cap B(z, r))^{c}$, then $\left|h_{r}(y)-h_{r}(x)\right|=\left|h_{r}(x)\right| \leqslant V(r) \leqslant 2 V(|x-y|)$ by 
subadditivity of $V$. If $y \in D \cap B(z, r),\left|h_{r}(y)-h_{r}(x)\right| \leqslant V\left(\left|\delta_{D}(y)-\delta_{D}(x)\right|\right) \leqslant V(|x-y|)$ by subadditivity of $V$. Hence by (1.4) and [6, Lemma 3.5], we obtain that

$$
|\mathrm{II}(x)| \leqslant 4 L_{0} \int_{r / 2 \leqslant|x-y|} V(|x-y|) \nu(|x-y|) d y \leqslant c_{5} V(r)^{-1} .
$$

From (4.3) and (4.4),

$$
\lim _{\varepsilon \downarrow 0} A_{\varepsilon}(x) \text { exists } \quad \text { and } \quad\left|\lim _{\varepsilon \downarrow 0} A_{\varepsilon}(x)\right| \leqslant\left(c_{3}+c_{5}\right) V(r)^{-1} .
$$

Finally from Proposition $3.4, \lim _{\varepsilon \downarrow 0} \mathcal{L}_{Z}^{\varepsilon} h_{r}(x)$ exists and

$$
\left|\lim _{\varepsilon \downarrow 0} \mathcal{L}_{Z}^{\varepsilon} h_{r}(x)\right| \leqslant C{ }_{3.4}^{V(r)^{-1}} .
$$

Hence combining (4.2), (4.5) and (4.6), we have the conclusion.

Theorem 4.2. For any $x \in D$, let $z_{x} \in \partial D$ be a point satisfying $\delta_{D}(x)=\left|x-z_{x}\right|$.

(1) There are constants $A_{4.2}=A_{4.2}\left(\phi, L_{0}, L_{3}, \Lambda, \eta\right) \in(0,1)$ and $C_{4.2}{ }_{1}=C 44_{4.2}{ }_{1}\left(\phi, L_{0}, L_{3}\right.$, $\Lambda, \eta)>0$, such that for any $s \leqslant A_{4.2} R_{0} / 2$ and $x \in D$ with $\delta_{D}(x)<s$,

$$
\mathbb{E}_{x}\left[\tau_{D \cap B\left(z_{x}, s\right)}\right] \leqslant C_{4.2}{ }_{1} V(s) V\left(\delta_{D}(x)\right) .
$$

(2) There is a constant $C_{4.2}{ }_{4}=C_{4.2}{ }_{2}\left(\phi, L_{0}, L_{3}, \Lambda, \eta\right)>0$, such that for any $s \leqslant R_{0} / 2$, $\lambda \geqslant 4$ and $x \in D$ with $\delta_{D}(x)<\lambda^{-1} s / 2$,

$$
\mathbb{P}_{x}\left(Y_{\tau_{D \cap B\left(z_{x}, \lambda-1 s\right)}} \in\left\{2 \Lambda|\widetilde{y}|<y_{d}, \lambda^{-1} s<|y|<\sin C S_{z_{x}}\right\}\right) \geqslant Q_{4.2} \frac{V\left(\delta_{D}(x)\right)}{V(s)} .
$$

Proof. Without loss of generality, we assume that $z_{x}=0$. For $R \leqslant R_{0} / 2$, let $h_{R}(y)=$ $V\left(\delta_{D}(y)\right) \mathbf{1}_{D \cap B(0, R)}(y)$. Let $f \geqslant 0$ be a smooth radial function such that $f(y)=0$ for $|y|>1$ and $\int_{\mathbb{R}^{d}} f(y) d y=1$. For $k \geqslant 1$, define $f_{k}(y):=2^{k d} f\left(2^{k} y\right)$ and $h_{R}^{(k)}:=f_{k} * h_{R} \in C_{c}^{2}\left(\mathbb{R}^{d}\right)$, and let $B_{k}^{\lambda}:=\left\{y \in D \cap B\left(0, \lambda^{-1} R / 4\right): \delta_{D \cap B\left(0, \lambda^{-1} R\right)}(y) \geqslant 2^{-k}\right\}$ for $\lambda \geqslant 4$.

Since $h_{R}^{(k)}$ is a $C_{c}^{2}$ function, $\mathcal{L} h_{R}^{(k)}$ is well defined everywhere. By Lemma 4.1, for $w \in B_{k}^{\lambda}$ and $u \in B\left(0,2^{-k}\right)$ the following limit

$$
\begin{aligned}
& \lim _{\varepsilon \downarrow 0} \int_{|w-y|>\varepsilon}\left(h_{R}(y-u)-h_{R}(w-u)\right) J(w, y) d y \\
= & \lim _{\varepsilon \downarrow 0} \int_{\left|(w-u)-y^{\prime}\right|>\varepsilon}\left(h_{R}\left(y^{\prime}\right)-h_{R}(w-u)\right) J\left(w, u+y^{\prime}\right) d y^{\prime}=\mathcal{L}^{u} h_{R}(w)
\end{aligned}
$$

exists and $-C_{4.1} V(R)^{-1} \leqslant \mathcal{L}^{u} h_{R}(w) \leqslant Q_{4.1} V(R)^{-1}$. We note that

$$
\begin{aligned}
& \int_{|w-y|>\varepsilon}\left(h_{R}^{(k)}(y)-h_{R}^{(k)}(w)\right) J(w, y) d y \\
= & \int_{|w-y|>\varepsilon} \int_{\mathbb{R}^{d}} f_{k}(u)\left(h_{R}(y-u)-h_{R}(w-u)\right) d u J(w, y) d y \\
= & \int_{|u|<2^{-k}} f_{k}(u) \int_{|w-y|>\varepsilon}\left(h_{R}(y-u)-h_{R}(w-u)\right) J(w, y) d y d u .
\end{aligned}
$$


By letting $\varepsilon \downarrow 0$ and using the dominated convergence theorem, it follows that for $w \in B_{k}^{\lambda}$ and $2^{-k+8}<\lambda^{-1} R$,

$$
\left|\mathcal{L} h_{R}^{(k)}(w)\right|=\left|\int_{|u|<2^{-k}} f_{k}(u) \mathcal{L}^{u} h_{R}(w) d u\right| \leqslant C_{4.1} V(R)^{-1} \int_{|u|<2^{-k}} f_{k}(u) d u=C 4_{4.1} V(R)^{-1} .
$$

Applying Lemma 2.2 to $B_{k}^{\lambda}$ and $h_{R}^{(k)}$, and using (4.9), for any $x \in B_{k}^{\lambda}$, we have

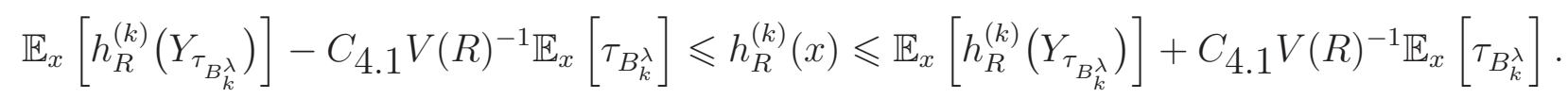

By letting $k \rightarrow \infty$, for any $x \in D \cap B\left(0, \lambda^{-1} R\right)$, we obtain

$$
\begin{aligned}
V\left(\delta_{D}(x)\right) & =h_{R}(x) \geqslant \mathbb{E}_{x}\left[h_{R}\left(Y_{\tau_{D \cap B(0, \lambda}-1} R\right)\right]-C \\
\text { and } V\left(\delta_{D}(x)\right) & =h_{R}(x) \leqslant \mathbb{E}_{x}\left[h_{R}\left(Y_{\tau_{D \cap B(0, \lambda}-1}\right)\right]+C(R)^{-1} \mathbb{E}_{x}\left[\tau_{D \cap B\left(0, \lambda^{-1} R\right)} V(R)^{-1} \mathbb{E}_{x}\left[\tau_{D \cap B\left(0, \lambda^{-1} R\right)}\right] .\right.
\end{aligned}
$$

For any $z \in D \cap B\left(0, \lambda^{-1} R\right)$ and $y \in D \cap\left(B(0, R) \backslash B\left(0, \lambda^{-1} R\right)\right)$, by the fact that $\nu$ is decreasing and (1.3) $), \nu(|y-z|) \geqslant \nu(2|y|) \geqslant c_{1} \nu(|y|)$. So by (1.4), (J1.1) and (4.1), we obtain

$$
\begin{aligned}
\mathbb{E}_{x}\left[h_{R}\left(Y_{\tau_{D \cap B(0, \lambda}-1}\right)\right] & \geqslant L_{0}^{-1} \mathbb{E}_{x} \int_{D \cap\left(B(0, R) \backslash B\left(0, \lambda^{-1} R\right)\right)} \int_{0}^{\tau_{D \cap B\left(0, \lambda^{-1} R\right)}} \nu\left(\left|Y_{t}-y\right|\right) d t h_{R}(y) d y \\
& \geqslant L_{0}^{-1} c_{1} \mathbb{E}_{x}\left[\tau_{D \cap B\left(0, \lambda^{-1} R\right)}\right] \int_{D \cap\left(B(0, R) \backslash B\left(0, \lambda^{-1} R\right)\right)} \nu(|y|) h_{R}(y) d y .
\end{aligned}
$$

Let $A:=\left\{\left(\widetilde{y}, y_{d}\right): 2 \Lambda|\widetilde{y}|<y_{d}\right\}$. For any $y \in A \cap B(0, R)$, since $y_{d}>2 \Lambda|\widetilde{y}|>2 \Lambda|\widetilde{y}|^{2}>\varphi(\widetilde{y})$, we have $A \cap B(0, R) \subset D \cap B(0, R)$ and

$$
\delta_{D}(y) \geqslant(1+\Lambda)^{-1}\left(y_{d}-\varphi(\widetilde{y})\right) \geqslant(2 \Lambda)^{-1}\left(y_{d}-\Lambda|\widetilde{y}|\right)>(4 \Lambda)^{-1} y_{d} \geqslant\left(4 \Lambda\left((2 \Lambda)^{-2}+1\right)^{1 / 2}\right)^{-1}|y| .
$$

Combining this and (3.4), $V\left(\delta_{D}(y)\right) \geqslant c_{2} V(|y|)$. By changing to polar coordinates with $|y|=t$, (3.3) and Proposition 3.1, we obtain that

$$
\begin{aligned}
& \int_{D \cap\left(B(0, R) \backslash B\left(0, \lambda^{-1} R\right)\right)} \nu(|y|) h_{R}(y) d y \geqslant c_{2} \int_{A \cap\left(B(0, R) \backslash B\left(0, \lambda^{-1} R\right)\right)} \nu(|y|) V(|y|) d y \\
& \geqslant c_{3} \int_{\lambda^{-1} R}^{R} \nu(t) V(t) t^{d-1} d t \geqslant c_{4} \int_{\lambda^{-1} R}^{R} V(t)^{-1} t^{-1} d t \\
& \geqslant c_{4} \cdot C_{3.1} \int_{\lambda^{-1} R}^{R} \frac{V^{\prime}(t)}{V(t)^{2}} d t=c_{4} \cdot C_{3.1}\left(V\left(\lambda^{-1} R\right)^{-1}-V(R)^{-1}\right) .
\end{aligned}
$$

Combining (4.12) and (4.13), there exists $C_{5}:=C_{5}\left(\underline{c}, \bar{\alpha}, \bar{C}, L_{0}, \Lambda, d\right)>0$ such that

$$
\mathbb{E}_{x}\left[h_{r}\left(Y_{\left.\tau_{D \cap B(0, \lambda-1}\right)}\right)\right] \geqslant C_{5} \mathbb{E}_{x}\left[\tau_{D \cap B\left(0, \lambda^{-1} R\right)}\right]\left(V\left(\lambda^{-1} R\right)^{-1}-V(R)^{-1}\right) .
$$

Using (3.4) again, $V\left(\lambda^{-1} R\right) \leqslant V\left(\lambda_{0}^{-1} R\right) \leqslant C_{V} \lambda_{0}^{-\underline{\alpha} / 2} V(R)$ for any $\lambda \geqslant \lambda_{0} \geqslant 4$. Let $\lambda_{0}:=$ $\left(2 C_{V}\left(C_{5}+C_{4.1}\right) / C_{5}\right)^{2 / \underline{\alpha}} \geqslant 1$. Then combining (4.10) and (4.14), we have that for $\lambda \geqslant \lambda_{0}$

$$
\begin{aligned}
V\left(\delta_{D}(x)\right) & \geqslant\left(C_{5} V\left(\lambda^{-1} R\right)^{-1}-\left(C_{5}+C_{4.1}\right) V(R)^{-1}\right) \mathbb{E}_{x}\left[\tau_{D \cap B\left(0, \lambda^{-1} R\right)}\right] \\
& \geqslant\left(C_{5} / 2\right) V\left(\lambda^{-1} R\right)^{-1} \mathbb{E}_{x}\left[\tau_{D \cap B\left(0, \lambda^{-1} R\right)}\right] .
\end{aligned}
$$


Thus, we have proved (4.7) with $A 4.2=\lambda_{0}^{-1}$ and $s=\lambda^{-1} R$ where $\lambda \geqslant \lambda_{0}$.

From (4.11) and Corollary 2.4 with (3.3) for $\delta_{D}(x)<\lambda^{-1} R / 2$ and $\lambda \geqslant 4$, we first note that

$$
\begin{aligned}
V\left(\delta_{D}(x)\right) & \leqslant V(R) \mathbb{P}_{x}\left(Y_{\left.\tau_{D \cap B(0, \lambda}-1 R\right)} \in D\right)+C[4.1 \\
& \leqslant\left(C(R)^{-1} \mathbb{E}_{x}\left[\tau_{D \cap B\left(0, \lambda^{-1} R\right)} V(R) V\left(\lambda^{-1} R\right)^{-2}+C 4.1\right]^{V(R)^{-1}}\right) \mathbb{E}_{x}\left[\tau_{D \cap B\left(0, \lambda^{-1} R\right)}\right] \\
& =c_{6} V(R)\left(V\left(\lambda^{-1} R\right)^{-2}+V(R)^{-2}\right) \mathbb{E}_{x}\left[\tau_{D \cap B\left(0, \lambda^{-1} R\right)}\right] .
\end{aligned}
$$

By (3.4) and the subadditivity of $V, V(s)^{-1} \geqslant 3^{-1}\left(3 \lambda^{-1} R / s\right)^{\underline{\alpha} / 2} C_{V}^{-1} V\left(\lambda^{-1} R\right)^{-1}$ for $s \leqslant 3 \lambda^{-1} R$. Combining this with (3.3) and using the polar coordinate with $|y|=t$, we have that

$$
\begin{aligned}
\int_{A \cap\left(B\left(0,3 \lambda^{-1} R\right) \backslash B\left(0,2 \lambda^{-1} R\right)\right)} \nu(|y|) d y & \geqslant c_{7} \int_{2 \lambda^{-1} R}^{3 \lambda^{-1} R} \nu(t) t^{d-1} d t \\
& \geqslant c_{8} \int_{2 \lambda^{-1} R}^{3 \lambda^{-1} R} V(t)^{-2} t^{-1} d t \geqslant c_{9} V\left(\lambda^{-1} R\right)^{-2} .
\end{aligned}
$$

For any $z \in B\left(0, \lambda^{-1} R\right)$ and $y \in B\left(0,3 \lambda^{-1} R\right) \backslash B\left(0,2 \lambda^{-1} R\right)$, by (1.3) and the fact that $\nu$ is decreasing, $\nu(|y-z|) \geqslant \nu(|y|+|z|) \geqslant \nu(3|y| / 2) \geqslant c_{10} \nu(|y|)$. So by (1.4), (J1.1) (4.1) and (4.18), we obtain

$$
\begin{aligned}
& \mathbb{P}_{x}\left(Y_{\left.\tau_{D \cap B(0, \lambda-1}\right)} \in A \cap\left(B\left(0,3 \lambda^{-1} R\right) \backslash B\left(0,2 \lambda^{-1} R\right)\right)\right) \\
\geqslant & L_{0}^{-1} \mathbb{E}_{x}\left[\int_{0}^{\left.\tau_{D \cap B(0, \lambda}-1 R\right)} \int_{A \cap\left(B\left(0,3 \lambda^{-1} R\right) \backslash B\left(0,2 \lambda^{-1} R\right)\right)} \nu\left(\left|Y_{s}-y\right|\right) d y d s\right] \\
\geqslant & L_{0}^{-1} c_{10} \mathbb{E}_{x}\left[\int_{0}^{\left.\tau_{D \cap B(0, \lambda}-1\right)} \int_{A \cap\left(B\left(0,3 \lambda^{-1} R\right) \backslash B\left(0,2 \lambda^{-1} R\right)\right)} \nu(|y|) d y d s\right] \\
\geqslant & c_{9} c_{10} L_{0}^{-1} V\left(\lambda^{-1} R\right)^{-2} \mathbb{E}\left[\tau_{\left.D \cap B\left(0, \lambda^{-1} R\right)\right] .}\right.
\end{aligned}
$$

Hence combining (4.17), (4.19), and the fact that $V$ is increasing, we conclude that for $\lambda \geqslant 4$,

$$
\begin{aligned}
V\left(\delta_{D}(x)\right) \leqslant c_{11} V(R)\left(V\left(\lambda^{-1} r\right)^{-2}+V(R)^{-2}\right) V\left(\lambda^{-1} R\right)^{2} & \\
\cdot & \mathbb{P}_{x}\left(Y_{\left.\tau_{D \cap B(0, \lambda-1}\right)} \in A \cap\left(B\left(0,3 \lambda^{-1} R\right) \backslash B\left(0,2 \lambda^{-1} R\right)\right)\right) \\
\leqslant 2 c_{11} V(R) \mathbb{P}_{x}\left(Y_{\tau_{D \cap B(0, \lambda}-1} \in\right) & \left.\in A \cap\left(B(0, R) \backslash B\left(0, \lambda^{-1} R\right)\right)\right) .
\end{aligned}
$$

Thus, we have proved (4.8) with $s=R$.

Remark 4.3.

\section{$5 \quad$ Upper bound estimates}

In this section, we derive the upper bound estimate on $p_{D}(t, x, y)$ for $t \leqslant T$ in $C^{1,1}$ open set $D$ with $C^{1,1}$ characteristics $\left(R_{0}, \Lambda\right)$. As before, we will assume that $R_{0}<1$ and $\Lambda>1$ and fix such $C^{1,1}$ open set $D$ throughout this section. We first introduce the next lemma which give a guideline to obtain the upper bound estimate on $p_{D}(t, x, y)$ (for its proof, see [18, Lemma 3.1] and [5, Lemma 1.10]). Applying (4.7) and Theorem 1.2 to (5.2), in Proposition 5.3 we will obtain the intermediate upper bound for $p_{D}(t, x, y)$ having one boundary decay. Applying this result, (4.7) and the upper bound of the survival probability of $Y$ (Lemma 5.2) to (5.1), we can get the short time sharp upper bound estimate for $p_{D}(t, x, y)$. 
Lemma 5.1. Let $Y$ be a symmetric pure jump Hunt process whose jumping intensity kernel $J$ satisfies the conditions $(\mathbf{J 1 . 1})$ and (J1.2). Suppose that $E \subset \mathbb{R}^{d}$ be an open set. Let $U_{1}, U_{3} \subset E$ be disjoint open subsets and $U_{2}:=E \backslash\left(U_{1} \cup U_{3}\right)$. If $x \in U_{1}, y \in U_{3}$ and $t>0$, we have

$$
\begin{aligned}
p_{E}(t, x, y) \leqslant & \mathbb{P}_{x}\left(Y_{\tau_{U_{1}}} \in U_{2}\right) \cdot \sup _{s<t, z \in U_{2}} p_{E}(s, z, y) \\
& +\int_{0}^{t} \mathbb{P}\left(\tau_{U_{1}}>s\right) \mathbb{P}_{y}\left(\tau_{E}>t-s\right) d s \cdot \sup _{u \in U_{1}, z \in U_{3}} J(u, z) \\
\leqslant & \mathbb{P}_{x}\left(Y_{\tau_{U_{1}}} \in U_{2}\right) \cdot \sup _{s<t, z \in U_{2}} p(s, z, y)+\left(t \wedge \mathbb{E}_{x}\left[\tau_{U_{1}}\right]\right) \cdot \sup _{u \in U_{1}, z \in U_{3}} J(u, z) .
\end{aligned}
$$

For the remainder of the section, we assume that $Y$ is the symmetric pure jump Hunt process with the jumping intensity kernel $J$ satisfying the conditions $(\mathbf{J 1 . 1}),(\mathbf{J} 1.2),\left(\mathbf{K}_{\eta}\right)$ and (SD). Let

$$
a_{T, R_{0}}:=\left[V\left(A_{4.2} R_{0} / 4\right)\right]^{2} T^{-1}
$$

where $A 4.2$ is the constant in Theorem $4.2(1)$. Denote $V^{-1}$ be the inverse function of $V$, then $V^{-1}\left(\sqrt{a_{T, R_{0}} \cdot t}\right) \leqslant A 4.2 R_{0} / 4$ for any $t \leqslant T$.

Lemma 5.2. There exists $C_{5.2}=C_{5.2}\left(\phi, L_{0}, L_{3}, \eta, R_{0}, \Lambda, T\right)$ such that for any $t \leqslant T$ and $x \in D$, we have that

$$
\mathbb{P}_{x}\left(\tau_{D}>t\right) \leqslant C_{5.2}\left(1 \wedge \frac{V\left(\delta_{D}(x)\right)}{\sqrt{t}}\right) .
$$

Proof. Let $r_{t}:=V^{-1}\left(\sqrt{a_{T, R_{0}} \cdot t}\right)$. We only consider the case $V\left(\delta_{D}(x)\right)<\sqrt{a_{T, R_{0}} \cdot t}$ which implies $\delta_{D}(x)<A A_{4.2} R_{0} / 4<R_{0} / 4$. Let $U_{t}:=D \cap B\left(z_{x}, 2 r_{t}\right) \subset D$ where $z_{x} \in \partial D$ with $\delta_{D}(x)=\left|x-z_{x}\right|$. Then, using Chebyshev's inequality and Corollary 2.4, we first obtain that

$$
\begin{aligned}
\mathbb{P}_{x}\left(\tau_{D}>t\right) & =\mathbb{P}_{x}\left(\tau_{U_{t}}>t, \tau_{D}=\tau_{U_{t}}\right)+\mathbb{P}_{x}\left(\tau_{D}>\tau_{U_{t}}>t\right) \\
& \leqslant \mathbb{P}_{x}\left(\tau_{U_{t}}>t\right)+\mathbb{P}_{x}\left(Y_{\tau_{U_{t}}} \in D\right) \leqslant\left(t^{-1}+C_{2.4} / \phi\left(2 r_{t}\right)\right) \mathbb{E}_{x} \tau_{U_{t}} .
\end{aligned}
$$

From (3.3) and the fact that $V$ is increasing and subadditive,

$$
\phi\left(2 r_{t}\right) \asymp\left[V\left(2 r_{t}\right)\right]^{2} \asymp\left[V\left(V^{-1}\left(\sqrt{a_{T, R_{0}} \cdot t}\right)\right]^{2}=a_{T, R_{0}} \cdot t .\right.
$$

Therefore, using (4.7) in Theorem 4.2, we conclude that

$$
\mathbb{P}_{x}\left(\tau_{D}>t\right) \leqslant c_{1} \frac{1}{t} \mathbb{E}_{x} \tau_{U_{t}} \leqslant c_{1} C_{4.2} 1 \frac{1}{t} V\left(r_{t}\right) V\left(\delta_{D}(x)\right) \leqslant c_{2} \frac{V\left(\delta_{D}(x)\right)}{\sqrt{t}} .
$$

We will use the following inequality several times, which follows from (WS): there exist $C_{I}:=\left(\bar{C} \underline{c}^{-1} \vee \underline{c}^{-2}\right)^{1 / \underline{\alpha}}>1$ such that

$$
C_{I}^{-1}\left(\frac{r}{R}\right)^{1 / \underline{\alpha}} \leqslant \frac{\phi^{-1}(r)}{\phi^{-1}(R)} \leqslant C_{I}\left(\frac{r}{R}\right)^{1 / \bar{\alpha}} \quad \text { for } 0<r \leqslant R .
$$

Recall the functions $F_{a, \gamma, T}(t, r)$ and $\Psi(t, x)$ are defined in (1.6) and (1.7), respectively.

Proposition 5.3. Let $a \leqslant a_{T, R_{0}}$. 
(1) Suppose that $D$ is bounded and the jumping intensity kernel $J$ satisfies the condition (J1). Then there exists a positive constant $C_{5.3}{ }_{1}=C_{5.3}\left(\phi, L_{0}, L_{3}, \eta, R_{0}, \Lambda, T, \operatorname{diam}(D), a\right)$ such that for any $(t, x, y) \in(0, T] \times D \times D$ with $V^{-1}(\sqrt{a \cdot t}) \leqslant|x-y|$, we have

$$
\left.p_{D}(t, x, y) \leqslant C_{5.3}\right]_{1} \Psi(t, x) \cdot\left(\left[\phi^{-1}(t)\right]^{-d} \wedge t \nu(|x-y|)\right) .
$$

(2) Suppose that the jumping intensity kernel J satisfies the condition (J2). Then there exists a positive constant $C_{5.3}=C_{5.3}\left(\beta, \phi, L_{0}, L_{3}, \eta, R_{0}, \Lambda, T, a\right)$ such that for any $(t, x, y) \in$ $(0, T] \times D \times D$ with $|x-y| \geqslant V^{-1}(\sqrt{a \cdot t}) \cdot \mathbf{1}_{\beta \in[0,1]}+2 \cdot \mathbf{1}_{\beta \in(1, \infty)}+\left(2+V^{-1}(\sqrt{a \cdot t})\right) \cdot \mathbf{1}_{\beta=\infty}$, we have

$$
p_{D}(t, x, y) \leqslant C_{[5.3]} \Psi(t, x) \cdot \begin{cases}F_{C_{1.2}^{\wedge \gamma_{1}, \gamma_{1}, T}}(t,|x-y| / 3) & \text { if } \beta \in[0, \infty), \\ (2 t / T|x-y|)^{9.2} & \text { if } \beta=\infty .\end{cases}
$$

Proof. Since we assume that $D$ is bounded in (1), by applying Theorem 1.1 instead of Theorem 1.2 the proof of (1) is similar to the that of (2), so we only give the proof of (2). Let $r_{t}:=$ $V^{-1}(\sqrt{a \cdot t}) / 9$. If $\delta_{D}(x) \geqslant r_{t} / 2$, using subadditivity of $V$, we see that $\Psi(t, x) \asymp 1$. Thus, by Theorem 1.2, and the fact that $r \rightarrow F_{c, \gamma, T}(t, r)$ is decreasing, we obtain the conclusion.

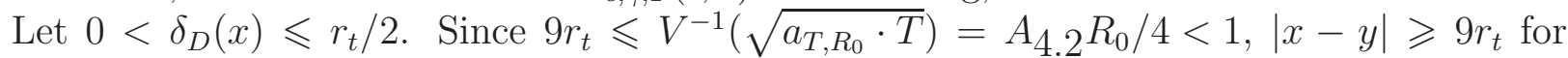
all $\beta \in[0, \infty]$. Choose a point $z_{x} \in \partial D$ with $\delta_{D}(x)=\left|x-z_{x}\right|$ and let $U_{1}:=B\left(z_{x}, r_{t}\right) \cap D$, $U_{3}:=\{z \in D:|z-x| \geqslant|x-y| / 2\}$ and $U_{2}:=D \backslash\left(U_{1} \cup U_{3}\right)$. Then $x \in U_{1}, y \in U_{3}$ and $U_{1} \cap U_{3}=\emptyset$. Note that $|x-y| / 2 \leqslant|x-y|-|z-x| \leqslant|y-z|$ for any $z \in U_{2}$. Therefore, by virtue of Theorem 1.2, we have we obtain

$$
\sup _{s<t, z \in U_{2}} p(s, z, y) \leqslant q_{1.2} \sup _{s<t,|z-y|>|x-y| / 2} F_{q_{1.2}^{\gamma_{1}, T}}(s,|z-y|) \leqslant c_{1} F_{q_{1.2}^{\gamma_{1}, T}}(t,|x-y| / 2) .
$$

In fact, if $\beta \in(1, \infty]$, we have $|z-y| \geqslant|x-y| / 2>1$ and so $F_{C{ }_{1.2}{ }^{\gamma_{1}, T}}(s,|z-y|)$ is increasing in $s$. If $\beta \in[0,1]$, we have $|z-y| \geqslant|x-y| / 2 \geqslant V^{-1}(\sqrt{a \cdot t}) / 2$ and so $\left(\left[\phi^{-1}(s)\right]^{-d} \wedge s \nu(\mid z-\right.$ $\left.y \mid) e^{-\gamma|z-y|^{\beta}}\right) \asymp s \nu(|z-y|) e^{-\gamma|z-y|^{\beta}}$ using (3.3), (3.4) and (5.3). Also, $s \nu(r) e^{-\gamma r^{\beta}}$ is increasing in $s$. Thus, combining there observations with the fact $r \rightarrow F_{{\left[\left[_{1.2} \gamma_{1}, T\right.\right.}}(t, r)$ is decreasing, the second inequality above holds.

By Corollary 2.4 and (4.7) in Theorem 4.2, we obtain

$$
\begin{aligned}
\mathbb{P}_{x}\left(Y_{\tau_{U_{1}}} \in U_{2}\right) & \leqslant \mathbb{P}_{x}\left(Y_{\tau_{U_{1}}} \in D\right) \leqslant q_{2.4} \phi\left(r_{t}\right)^{-1} \mathbb{E}_{x} \tau_{U_{1}} \\
& \leqslant q_{2.4} \cdot C_{4.2}{ }_{1} V\left(\delta_{D}(x)\right) / V\left(r_{t}\right) \leqslant c_{3} V\left(\delta_{D}(x)\right) / \sqrt{t} .
\end{aligned}
$$

In the last inequality we use monotonicity and subadditivity of $V$, which imply $V\left(r_{t}\right) \asymp \sqrt{t}$.

Note that for $u \in U_{1}$ and $z \in U_{3}$ that

$$
|u-z| \geqslant|z-x|-\left|x-z_{x}\right|-\left|u-z_{x}\right| \geqslant|x-y| / 2-3 r_{t} / 2 .
$$

Let $\beta \in[0, \infty)$. Since $|x-y| \geqslant 9 r_{t}$, from (5.6) we have $|u-z| \geqslant|x-y| / 3$ for $(u, z) \in U_{1} \times U_{3}$, therefore by (1.4), (1.5) and (J2),

$$
\left(\sup _{u \in U_{1}, z \in U_{3}} J(u, z)\right) \leqslant c_{4} e^{-\gamma_{1}(|x-y| / 3)^{\beta}} \nu(|x-y|) \leqslant c_{5} t^{-1} F_{\gamma_{1}, \gamma_{1}, T}(t,|x-y| / 3) .
$$


Combining this with (4.7) in Theorem 4.2, we conclude that

$$
\begin{aligned}
\mathbb{E}_{x}\left[\tau_{U_{1}}\right]\left(\sup _{u \in U_{1}, z \in U_{3}} J(u, z)\right) & \leqslant C 4.2]_{1} V(r) V\left(\delta_{D}(x)\right) \cdot c_{5} t^{-1} F_{\gamma_{1}, \gamma_{1}, T}(t,|x-y| / 3) \\
& \leqslant c_{6} \frac{V\left(\delta_{D}(x)\right)}{\sqrt{t}} F_{\gamma_{1}, \gamma_{1}, T}(t,|x-y| / 3) .
\end{aligned}
$$

If $\beta=\infty$, since $|u-z| \geqslant|x-y| / 2-3 r_{t} / 2 \geqslant\left(1+4 r_{t}\right)-3 r_{t} / 2 \geqslant 1$, we have $J(u, z)=0$.

Hence, by applying (5.4) (5.7) to (5.2) for the case $\beta \in[0, \infty)$ and by applying (5.7) to (5.2) for the case $\beta=\infty$, we reach the conclusion.

We denote by $X$ the process in the case $\beta=0$ in ( $\mathbf{J 2})$, that is, $X$ is a symmetric Hunt process whose jumping kernel is $J^{X}(x, y):=\kappa(x, y) \nu(|x-y|)$.

Proposition 5.4. (1) Suppose that the jumping intensity kernel $J$ satisfying (J1) and $D$ is bounded. There exists a positive constant 95.4$]_{1}=C_{5.4}\left(\phi, L_{0}, L_{3}, \eta, R_{0}, \Lambda, T, \operatorname{diam}(D)\right)$ such that for any $(t, x, y) \in(0, T] \times D \times D$, we have

$$
p_{D}(t, x, y) \leqslant C[5.4]_{1} \Psi(t, x) \Psi(t, y)\left(\left[\phi^{-1}(t)\right]^{-d} \wedge t \nu(|x-y|)\right) .
$$

(2) There exists a positive constant $C_{5.4}=C_{5.4}\left(\phi, L_{0}, L_{3}, \eta, R_{0}, \Lambda, T\right)$ such that for any $(t, x, y) \in$ $(0, T] \times D \times D$, we have

$$
p_{D}^{X}(t, x, y) \leqslant 9_{5.4} \Psi(t, x) \Psi(t, y)\left(\left[\phi^{-1}(t)\right]^{-d} \wedge t \nu(|x-y|)\right) .
$$

Proof. Using Theorem 1.1 and Proposition 5.3 (1) instead of Theorem 1.2 and Proposition 5.3 (2) respectively, the proof of (1) is almost identical to the one of (2). So we only give the proof of $(2)$.

The semigroup property, Theorem 1.2 (for $\beta=0$ ), (5.3) and Lemma 5.2 yield

$$
\begin{aligned}
p_{D}^{X}(t / 2, x, y) & \leqslant\left(\sup _{z, w \in D} p_{D}^{X}(t / 4, z, w)\right) \int_{D} p_{D}^{X}(t / 4, x, z) d z \\
& \leqslant c_{1}\left[\phi^{-1}(t / 4)\right]^{-d} \mathbb{P}_{x}\left(\tau_{D}>t / 4\right) \leqslant c_{2}\left[\phi^{-1}(t)\right]^{-d} \Psi(t, x) .
\end{aligned}
$$

Thus, by Proposition 5.3, (5.3) and Theorem 1.2 (for $\beta=0$ ), we obtain

$$
p_{D}^{X}(t / 2, x, y) \leqslant c_{3} \Psi(t / 2, x)\left(\left[\phi^{-1}(t / 2)\right]^{-d} \wedge(t / 2) \nu(|x-y|)\right) \leqslant c_{4} \Psi(t, x) p^{X}(t / 2, x, y) .
$$

Combining these with Theorem 1.2 (for $\beta=0$ ), the symmetry $p_{D}^{X}$ and the semigroup property of $p^{X}$, we conclude that

$$
\begin{aligned}
p_{D}^{X}(t, x, y) & =\int_{D} p_{D}^{X}(t / 2, x, z) \cdot p_{D}^{X}(t / 2, z, y) d z \leqslant c_{4}^{2} \Psi(t, x) \Psi(t, y) p^{X}(t, x, y) \\
& \leqslant c_{5} \Psi(t, x) \Psi(t, y)\left(\left[\phi^{-1}(t)\right]^{-d} \wedge t \nu(|x-y|)\right) .
\end{aligned}
$$

Suppose that the jumping intensity kernel $J$ satisfying (J2). By Meyer's construction (e.g., see [19, §4.1]), when $\beta \in(0, \infty]$ the process $Y$ can be constructed from $X$ by removing jumps of size greater than 1 with suitable rate. Let $p_{D}^{X}(t, x, y)$ be the transition density function of $X$ on $D$. For $\beta \in(0, \infty]$, we define

$$
\mathcal{J}(x):=\int_{\mathbb{R}^{d}} \kappa(x, y) \nu(|x-y|)\left(1-\chi(|x-y|)^{-1}\right) d y
$$


where $\chi(|x-y|)$ is defined in (1.5). Then $\|\mathcal{J}\|_{\infty} \leqslant c_{1} \int_{|z| \geqslant 1} \nu(|z|) d z<\infty$. By [1, Lemma 3.6] we have

$$
p_{D}(t, x, y) \leqslant e^{T\|\mathcal{J}\|_{\infty}} p_{D}^{X}(t, x, y) \quad \text { for any }(t, x, y) \in(0, T] \times D \times D .
$$

Thus, the sharp upper bound of of $p_{D}(t, x, y)$ for $|x-y|<M$ for some $M>0$ follows from the one of $p_{D}^{X}(t, x, y)$ and (5.8). Therefore Combining (5.8), Propositions 5.3)(2) and 5.4(2), we have the following result.

Proposition 5.5. Suppose that the jumping intensity kernel J satisfying (J2). There exists a positive constant $C_{5.5}=C_{5.5}\left(\beta, \phi, L_{0}, L_{3}, \eta, R_{0}, \Lambda, T\right)$ such that for every $(t, x, y) \in(0, T] \times$ $D \times D$ we have

$$
p_{D}(t, x, y) \leqslant G_{[5.5} \Psi(t, x) \cdot \begin{cases}F_{q_{1.2}^{\wedge \gamma_{1}, \gamma_{1}, T}}(t,|x-y| / 3) & \text { if } \beta \in[0, \infty), \\ F_{q_{1.2} \gamma^{\gamma_{1}, T}}(t,|x-y| / 2) & \text { if } \beta=\infty,\end{cases}
$$

where $G_{1.2}$ is the constant in Theorem 1.2 and $\gamma_{1}$ is the constant in (1.5).

Now we are ready to prove the upper bound of Theorem 1.3(1) and Theorem 1.5(1).

Proofs of the upper bounds of $p_{D}(t, x, y)$ in Theorems 1.3(1) and 1.5(1). In Proposition 5.5)(1), we have proved the upper bound of $p_{D}(t, x, y)$ in Theorem 1.3)(1). So we only give the proof of the upper bound of $p_{D}(t, x, y)$ in Theorem 1.5)(1).

Let $r_{t}:=V^{-1}\left(\sqrt{a_{T, R_{0}} \cdot t}\right)$ so that $r_{t} \leqslant A_{4.2} R_{0} / 4<1 / 4$. By Proposition 5.5 and the symmetry of $p_{D}(t, x, y)$, we only need to prove the upper bound of $p_{D}(t, x, y)$ for the case $\delta_{D}(x) \vee \delta_{D}(y)<r_{t}$, which we will assume throughout the proof.

If $\beta=\infty$ and $6<|x-y| \leqslant 6\left(1 \vee C_{[1.2}^{-1}\right)$, by (5.8) and Proposition [5.4, we have

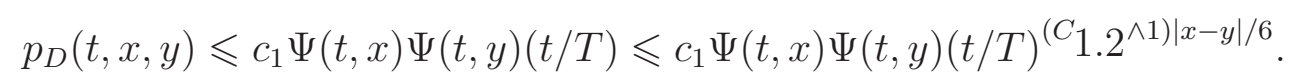

If either the case $\beta \in[0, \infty)$ and $|x-y| \leqslant 6\left(1 \vee C_{1.2}^{-1}\right)$ holds or the case $\beta=\infty$ and $|x-y| \leqslant 6$ holds, by (5.8) and Proposition 5.4(2), we have

$$
p_{D}(t, x, y) \leqslant e^{T\|\mathcal{J}\|_{\infty}} p_{D}^{X}(t, x, y) \leqslant c_{2} \Psi(t, x) \Psi(t, y)\left(\left[\phi^{-1}(t)\right]^{-d} \wedge t \nu(|x-y|)\right) .
$$

Thus, the upper bound of $p_{D}(t, x, y)$ in Theorem 1.5)(1) holds for $|x-y| \leqslant 6\left(1 \vee C_{1.2}^{-1}\right)$.

For the remainder of the proof, we assume that $\delta_{D}(x) \vee \delta_{D}(y)<r_{t}$ and $|x-y|>6\left(1 \vee C_{[1.2}^{-1}\right)$. For any $x$ with $\delta_{D}(x)<r_{t}$, let $z_{x} \in \partial D$ such that $\delta_{D}(x)=\left|z_{x}-x\right|$. Let $U_{1}:=B\left(z_{x}, r_{t}\right) \cap D$, $U_{3}:=\{z \in D:|z-x| \geqslant|x-y| / 2\}$, and $U_{2}:=D \backslash\left(U_{1} \cup U_{3}\right)$. Note that $x \in U_{1}$ and $y \in U_{3}$ and $|x-y| / 2 \leqslant|z-y|$ for $z \in U_{2}$. Thus, by Proposition 5.5 we have

$$
\begin{aligned}
& \sup _{s<t, z \in U_{2}} p_{D}(s, z, y) \\
& \leqslant \sup _{s<t, z \in U_{2}} q_{5.5} \frac{V\left(\delta_{D}(y)\right)}{\sqrt{s}} \cdot\left(F_{\mathcal{C 1 . 2}^{\wedge \gamma_{1}, \gamma_{1}, T}}(s,|z-y| / 3) \cdot \mathbf{1}_{\beta \in[0, \infty)}+F_{\mathbb{1 . 2}^{\gamma_{1}, T}}(s,|z-y| / 2) \cdot \mathbf{1}_{\beta=\infty}\right) \\
& \leqslant C_{5.5} V\left(\delta_{D}(y)\right) \sup _{s<t,|x-y| / 2 \leqslant|z-y|} \frac{1}{\sqrt{s}} \\
& \cdot\left(F_{Q_{1.2}^{\wedge \gamma_{1}, \gamma_{1}, T}}(s,|z-y| / 3) \cdot \mathbf{1}_{\beta \in[0, \infty)}+F_{\left[_{1.2}^{\gamma_{1}, T}\right.}(s,|z-y| / 2) \cdot \mathbf{1}_{\beta=\infty}\right) \\
& \leqslant c_{3} \frac{V\left(\delta_{D}(y)\right)}{\sqrt{t}} \cdot\left(F_{q_{1.2}^{\wedge \gamma_{1}, \gamma_{1}, T}}(t,|x-y| / 6) \cdot \mathbf{1}_{\beta \in[0, \infty)}+F_{q_{1.2}^{\gamma_{1}, T}}(t,|x-y| / 4) \cdot \mathbf{1}_{\beta=\infty}\right) .
\end{aligned}
$$


The last inequality is clear for $\beta \in[0, \infty)$ by the definition of $F_{q_{1.2}{ }^{\gamma_{1}, \gamma_{1}, T}}(t, r)$ and for $\beta=\infty$ we used the fact that $s \rightarrow s^{-1 / 2}(s / T r)^{a r}$ is increasing if $a r \geqslant 1$. Hence from (5.5) and (5.10), we obtain

$$
\begin{aligned}
& \mathbb{P}_{x}\left(Y_{\tau_{U_{1}}} \in U_{2}\right)\left(\sup _{s<t, z \in U_{2}} p_{D}(s, z, y)\right) \\
& \leqslant c_{4} \frac{V\left(\delta_{D}(x)\right)}{\sqrt{t}} \frac{V\left(\delta_{D}(y)\right)}{\sqrt{t}} \cdot \begin{cases}F_{Q_{1.2}{ }^{\gamma_{1}, \gamma_{1}, T}}(t,|x-y| / 6) & \text { if } \beta \in[0, \infty), \\
F_{Q_{1.2} r^{\gamma_{1}, T}}(t,|x-y| / 4) & \text { if } \beta=\infty .\end{cases}
\end{aligned}
$$

Also from Lemma 5.2, we have

$$
\begin{aligned}
& \int_{0}^{t} \mathbb{P}_{x}\left(\tau_{U_{1}}>s\right) \mathbb{P}_{y}\left(\tau_{D}>t-s\right) d s \leqslant \int_{0}^{t} \mathbb{P}_{x}\left(\tau_{D}>s\right) \mathbb{P}_{y}\left(\tau_{D}>t-s\right) d s \\
& \quad \leqslant C_{5.2}^{2} V\left(\delta_{D}(x)\right) V\left(\delta_{D}(y)\right) \int_{0}^{t} s^{-1 / 2}(t-s)^{-1 / 2} d s \leqslant c_{5} t \frac{V\left(\delta_{D}(x)\right)}{\sqrt{t}} \frac{V\left(\delta_{D}(y)\right)}{\sqrt{t}} .
\end{aligned}
$$

For $(u, z) \in U_{1} \times U_{3}$ and $|x-y|>6>6 r_{t}$, note that $|u-z| \geqslant|x-y|-|x-u|-|z-y| \geqslant|x-y| / 3$. Thus, if $\beta \in[0, \infty)$, by (1.5) and (J2),

$$
\left(\sup _{u \in U_{1}, z \in U_{3}} J(u, z)\right) \leqslant c_{6} e^{-\gamma_{1}(|x-y| / 3)^{\beta}} \nu(|x-y| / 3) \leqslant c_{7} t^{-1} F_{\gamma_{1}, \gamma_{1}, T}(t,|x-y| / 3) .
$$

Combining this with (5.12), we obtain

$$
\begin{aligned}
& \int_{0}^{t} \mathbb{P}_{x}\left(\tau_{U_{1}}>s\right) \mathbb{P}_{y}\left(\tau_{D}>t-s\right) d s \cdot\left(\sup _{u \in U_{1}, z \in U_{3}} J(u, z)\right) \\
& \leqslant c_{8} \frac{V\left(\delta_{D}(x)\right)}{\sqrt{t}} \frac{V\left(\delta_{D}(y)\right)}{\sqrt{t}} F_{\gamma_{1}, \gamma_{1}, T}(t,|x-y| / 3) .
\end{aligned}
$$

If $\beta=\infty$, since $|u-z|>1, J(u, z)=0$ on $U_{1} \times U_{3}$.

Therefore by applying (5.11) and (5.13) for $\beta \in[0, \infty)$ and by applying (5.11) for $\beta=$ $\infty$ in (5.1) of Lemma 5.1, we prove the upper bound of $p_{D}(t, x, y)$ in Theorem 1.5(1) for $\delta_{D}(x) \vee \delta_{D}(y)<r_{t}$ and $|x-y|>6\left(1 \vee C_{[1.2}^{-1}\right)$.

\section{$6 \quad$ Preliminary lower bound estimates}

In this section, we discuss a preliminary lower bound for $p_{D}(t, x, y)$. In this section we will always assume that $Y$ is the symmetric pure jump Hunt process with the jumping intensity kernel $J$ satisfying either the conditions $(\mathbf{J} 1.2)$ and $(\mathbf{J 1 . 3})$ or the condition $(\mathbf{J} 2)$.

Since $Y$ satisfies conditions imposed in [11, using [11, Theorem 5.2 and Lemma 2.5], the proof of the next lemma is the same as that of [17, Lemma 3.2]. Thus, we omit the proof.

Lemma 6.1. Let $a, b$ and $T$ be positive constants. Then there exists a constant $C_{6.1}=C_{6.1}$ $\left(a, b, L_{0}, \phi, T\right)>0$ such that for all $\lambda \in(0, T]$ we have

$$
\inf _{\substack{y \in \mathbb{R}^{d} \\|y-z| \leqslant \phi^{-1}(\lambda)}} \mathbb{P}_{y}\left(\tau_{B\left(z, 2 b \phi^{-1}(\lambda)\right)}>a \lambda\right) \geqslant C[6.1
$$


Let $D$ be an arbitrary non-empty open set, and $a$ and $T$ be positive constants. We use the convention that $\delta_{D}(\cdot) \equiv \infty$ when $D=\mathbb{R}^{d}$ to derive the lower bound of $p(t, x, y)$ in Theorem 1.1 and 1.2 simultaneously.

Using [11, Theorem 5.2] and Lemma 6.1, the proof of the following Proposition is similar to that of [17, Proposition 3.3]. Thus, we omit the proof.

Proposition 6.2. Let $D$ be an arbitrary open set and let a and $T$ be positive constants. Suppose that $(t, x, y) \in(0, T] \times D \times D$, with $\delta_{D}(x) \geqslant a \phi^{-1}(t) \geqslant 2|x-y|$. Then there exists a positive constant $C_{6.2}=C_{6.2}\left(a, L_{0}, \phi, T\right)$ such that $p_{D}(t, x, y) \geqslant C_{6.2}\left[\phi^{-1}(t)\right]^{-d}$.

From Lemma 6.1 and Proposition 6.2 we see that, under the condition (WS) on $\phi$, the behavor of $Y$ is locally stable in terms of $\phi$.

Proposition 6.3. Let $D$ be an arbitrary open set and let a and $T$ be positive constants.

(1) Suppose that the jumping intensity kernel $J$ satisfies the conditions (J1.2) and (J1.3). Then for every $M>0$, there exists a constant $C_{6.3}{ }_{1}=C_{6.3}{ }_{1}\left(a, M, L_{0}, \phi, T\right)>0$ such that for all $(t, x, y) \in(0, T] \times D \times D$ with $\delta_{D}(x) \wedge \delta_{D}(y) \geqslant a \phi^{-1}(t)$ and $a \phi^{-1}(t) \leqslant$ $2|x-y| \leqslant 2 M$ we have $p_{D}(t, x, y) \geqslant C\left[6.3{ }_{1} t \nu(|x-y|)\right.$.

(2) Suppose that the jumping intensity kernel J satisfies the condition (J2). Then there exists a constant $C_{6.3}=C_{6.3}\left(a, L_{0}, \phi, T\right)>0$ such that for every $(t, x, y) \in(0, T] \times D \times D$, with $\delta_{D}(x) \wedge \delta_{D}(y) \geqslant a \phi^{-1}(t)$ and $a \phi^{-1}(t) \leqslant 2|x-y|$, we have $p_{D}(t, x, y) \geqslant C 6.3 t \nu(\mid x-$ $y \mid) / \chi(|x-y|)$.

Proof. We first give the proof of (2). By Lemma 6.1, there exists $c_{1}=c_{1}\left(a, L_{0}, \phi, T\right)>0$ such that

$$
\inf _{\left\{|z-y| \leqslant 4^{-1} a \phi^{-1}(t)\right\}} \mathbb{P}_{z}\left(\tau_{B\left(z, 6^{-1} a \phi^{-1}(t)\right)}>t\right) \geqslant c_{1} .
$$

Thus by the strong Markov property

$$
\mathbb{P}_{x}\left(Y_{t}^{D} \in B\left(y, 2^{-1} a \phi^{-1}(t)\right)\right) \geqslant c_{1} \mathbb{P}_{x}\left(Y^{D} \text { hits the ball } B\left(y, 4^{-1} a \phi^{-1}(t)\right) \text { by time } t\right) .
$$

Using this and the Lévy system in (4.1), we obtain

$$
\begin{aligned}
& \mathbb{P}_{x}\left(Y_{t}^{D} \in B\left(y, 2^{-1} a \phi^{-1}(t)\right)\right) \\
\geqslant & c_{1} \mathbb{P}_{x}\left(Y_{\left.t \wedge \tau_{B\left(x, 6^{-1} a \phi^{-1}(t)\right)}^{D} \in B\left(y, 4^{-1} a \phi^{-1}(t)\right) \text { and } t \wedge \tau_{B\left(x, 6^{-1} a \phi^{-1}(t)\right)} \text { is a jumping time }\right)} J\left(Y_{s}, u\right) d u d s\right] . \\
= & c_{1} \mathbb{E}_{x}\left[\int_{B\left(y, 4^{-1} a \phi^{-1}(t)\right)}^{t \wedge \tau_{B\left(x, 6 \cdot 2^{-5} a \phi^{-1}(t)\right)}} \int_{0}\right.
\end{aligned}
$$

Lemma 6.1 also implies that

$$
\mathbb{E}_{x}\left[t \wedge \tau_{B\left(x, 6 \cdot 2^{-5} a \phi^{-1}(t)\right)}\right] \geqslant t \mathbb{P}_{x}\left(\tau_{B\left(x, 6 \cdot 2^{-5} a \phi^{-1}(t)\right)} \geqslant t\right) \geqslant c_{2} t \quad \text { for all } t \in(0, T] .
$$

Let $w$ be the point on the line connecting $x$ and $y$ (i.e., $|x-y|=|x-w|+|w-y|$ ) such that $|w-y|=7 \cdot 2^{-5} a \phi^{-1}(t)$, then $B\left(w, 2^{-5} a \phi^{-1}(t)\right) \subset B\left(y, 4^{-1} a \phi^{-1}(t)\right)$. Moreover, for every $(z, u) \in B\left(x, 6 \cdot 2^{-5} a \phi^{-1}(t)\right) \times B\left(w, 2^{-5} a \phi^{-1}(t)\right)$, we have

$$
\begin{aligned}
|z-u| & <6^{-1} a \phi^{-1}(t)+2^{-5} a \phi^{-1}(t)+|x-w| \\
& =|x-y|+\left(6 \cdot 2^{-5}+2^{-5}-7 \cdot 2^{-5}\right) a \phi^{-1}(t)=|x-y|
\end{aligned}
$$


and thus $B\left(w, 2^{-5} a \phi^{-1}(t)\right) \subset\{u:|z-u|<|x-y|\}$. Combining this result with (J2), (1.4) and (6.3), we obtain

$$
\begin{aligned}
& \mathbb{E}_{x}\left[\int_{0}^{t \wedge \tau_{B\left(x, 6 \cdot 2^{-5} a \phi^{-1}(t)\right)}} \int_{B\left(y, 4^{-1} a \phi^{-1}(t)\right)} J\left(Y_{s}, u\right) d u d s\right] \\
\geqslant & \mathbb{E}_{x}\left[\int_{0}^{t \wedge \tau_{B\left(x, 6 \cdot 2^{-5} a \phi^{-1}(t)\right)}} \int_{B\left(w, 2^{-5} a \phi^{-1}(t)\right)} J\left(Y_{s}, u\right) \mathbf{1}_{\left\{\left|Y_{s}-u\right|<|x-y|\right\}} d u d s\right] \\
\geqslant & L_{0}^{-1} \mathbb{E}_{x}\left[t \wedge \tau_{\left.B\left(x, 6 \cdot 2^{-5} a \phi^{-1}(t)\right)\right]\left|B\left(w, 2^{-5} a \phi^{-1}(t)\right)\right| \nu(|x-y|) / \chi(|x-y|)}\right. \\
> & c_{3} t\left[\phi^{-1}(t)\right]^{d} \nu(|x-y|) / \chi(|x-y|) .
\end{aligned}
$$

Then, using the semigroup property along with Proposition 6.2, (6.4) and (5.3), the proposition follows from the proof of [17, Proposition 3.5].

The proof of (1) is identical to the that of (2) except that we apply (J1.3) in (6.4) instead of (J2) and (1.4).

Combining Propositions 6.2 and 6.3. we obtain the following preliminary lower bound of $p_{D}(t, x, y)$. Note that the lower bound in Proposition 6.4(1) is the sharp interior lower bound of $p_{D}(t, x, y)$ under the conditions (J1.2) and (J1.3). Moreover, under the condition (J2), the lower bound in Proposition 6.4(2) that yields the sharp interior lower bound of $p_{D}(t, x, y)$ for the case $\beta \in[0,1]$ and the case $\beta \in(1, \infty]$ with $|x-y|<1$.

Proposition 6.4. Let $D$ be an arbitrary open set and let a and $T$ be positive constants.

(1) Suppose that the jumping intensity kernel $J$ satisfies the conditions (J1.2) and (J1.3).

Then, for every $(t, x, y) \in(0, T] \times D \times D$ and $M>0$, with $\delta_{D}(x) \wedge \delta_{D}(y) \geqslant a \phi^{-1}(t)$ and $|x-y|<M$, there exists a constant $C_{6.4]_{1}}=C_{6.4}\left(a, M, L_{0}, \phi, T\right)>0$ such that

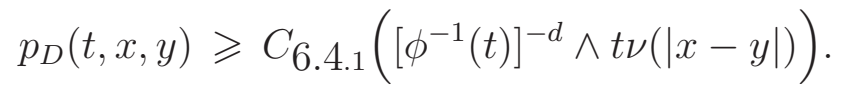

(2) Suppose that the jumping intensity kernel $J$ satisfies the condition (J2). Then, for every $(t, x, y) \in(0, T] \times D \times D$, with $\delta_{D}(x) \wedge \delta_{D}(y) \geqslant a \phi^{-1}(t)$, there exists a constant $46.4=$ $C_{6.4}\left(a, L_{0}, \phi, T\right)>0$ such that

$$
p_{D}(t, x, y) \geqslant C\left[6.4\left(\left[\phi^{-1}(t)\right]^{-d} \wedge t \nu(|x-y|) / \chi(|x-y|)\right) .\right.
$$

For the remainder of this section, assume that the jumping intensity kernel $J$ satisfies the condition (J2) for $\beta \in(1, \infty]$ with $|x-y| \geqslant 1$. Also, we assume that $D$ is an connected open set with the following property: there exist $\lambda_{1} \in[1, \infty)$ and $\lambda_{2} \in(0,1]$ such that for every $r \leqslant 1$ and $x, y$ in the same component of $D$ with $\delta_{D}(x) \wedge \delta_{D}(y) \geqslant r$ there exists in $D$ a length parameterized rectifiable curve $l$ connecting $x$ to $y$ with the length $|l|$ of $l$ less than or equal to $\lambda_{1}|x-y|$ and $\delta_{D}(l(u)) \geqslant \lambda_{2} r$ for $u \in[0,|l|]$.

Now we prove the preliminary lower bound of $p_{D}(t, x, y)$ separately for the case $\beta=\infty$ and the case $\beta \in(1, \infty)$. We will closely follow the proofs of [10, Theorem 3.6] and [12, Theorem $5.5]$.

Proposition 6.5. Let $\beta=\infty$. Suppose that $T>0$ and $a \in\left(0,\left(4 \phi^{-1}(T)\right)^{-1}\right]$. Then there exist constants $\left.C_{6.5}\right]_{i}=C_{6.5}\left(a, L_{0}, \phi, T, \lambda_{1}, \lambda_{2}\right)>0, i=1,2$, such that for any $x, y \in D$ with $\delta_{D}(x) \wedge \delta_{D}(y) \geqslant a \phi^{-1}(t),|x-y| \geqslant 1$, and $t \leqslant T$ we have

$$
p_{D}(t, x, y) \geqslant C_{6.5}\left(\frac{t}{T|x-y|}\right)^{\left\lceil[6.5]_{2}|x-y|\right.} .
$$


Proof. Let $R_{1}:=|x-y| \geqslant 1$, and by the assumption on $D$, there is a length parameterized curve $l \subset D$ connecting $x$ and $y$ such that the total length $|l| \leqslant \lambda_{1} R_{1}$ and $\delta_{D}(l(u)) \geqslant \lambda_{2} a \phi^{-1}(t)$ for every $u \in[0,|l|]$. Define $k$ be the integer satisfying $(4 \leqslant) 4 \lambda_{1} R_{1} \leqslant k<4 \lambda_{1} R_{1}+1 \leqslant 5 \lambda_{1} R_{1}$ and $r_{t}:=2^{-1} \lambda_{2} a \phi^{-1}(t) \leqslant 8^{-1}$. For each $i=0,1,2, \ldots, k$, let $x_{i}:=l(i|l| / k)$ and $B_{i}:=B\left(x_{i}, r_{t}\right)$, then $\delta_{D}\left(x_{i}\right) \geqslant 2 r_{t}$ and $B_{i} \subset B\left(x_{i}, 2 r_{t}\right) \subset D$. Since $4 \lambda_{1} R_{1} \leqslant k$ for each $y_{i} \in B_{i}$, we have

$$
\left|y_{i}-y_{i+1}\right| \leqslant\left|y_{i}-x_{i}\right|+\left|x_{i}-x_{i+1}\right|+\left|x_{i+1}-y_{i+1}\right| \leqslant \frac{1}{8}+\frac{|l|}{k}+\frac{1}{8} \leqslant \frac{\lambda_{1} R_{1}}{4 \lambda_{1} R_{1}}+\frac{1}{4}=\frac{1}{2} .
$$

Moreover $\delta_{D}\left(y_{i}\right) \geqslant \delta_{D}\left(x_{i}\right)-\left|y_{i}-x_{i}\right| \geqslant r_{t} \geqslant r_{t / k}$. Thus by Proposition 6.4(2), there are constants $c_{i}=c_{i}\left(a, L_{0}, \phi, T\right)>0, i=1,2$, such that for $\left(y_{i}, y_{i+1}\right) \in B_{i} \times B_{i+1}$ we have

$$
p_{D}\left(t / k, y_{i}, y_{i+1}\right) \geqslant c_{1}\left(\frac{1}{\left[\phi^{-1}(t / k)\right]^{d}} \wedge \frac{t / k}{\phi\left(\left|y_{i}-y_{i+1}\right|\right)\left|y_{i}-y_{i+1}\right|^{d}}\right) \geqslant c_{2} t /(T k) .
$$

The last inequality comes from $t / k \leqslant T / 4$ for the first part and (6.5) for the second part. Note that $r_{t} \geqslant c_{3}(t / k T)^{1 / \underline{\alpha}}$ for some $c_{3}=c_{3}\left(a, \phi, T, \lambda_{2}\right)$ by (5.3). Hence, combining these observations and the fact that $k \asymp R_{1}$, we conclude that

$$
\begin{aligned}
& p_{D}(t, x, y) \geqslant \int_{B_{1}} \ldots \int_{B_{k-1}} p_{D}\left(t / k, x, y_{1}\right) \ldots p_{D}\left(t / k, y_{k-1}, y\right) d y_{k-1} \ldots d y_{1} \\
& \geqslant\left(c_{2} t(T k)^{-1}\right)^{k} \Pi_{i=1}^{k-1}\left|B_{i}\right| \geqslant\left(c_{4} t(T k)^{-1}\right)^{c_{5} k} \geqslant\left(c_{6} t\left(T R_{1}\right)^{-1}\right)^{c_{7} R_{1}} \geqslant c_{8}\left(t\left(T R_{1}\right)^{-1}\right)^{c_{9} R_{1}} .
\end{aligned}
$$

Proposition 6.6. Let $\beta \in(1, \infty)$. Suppose that $T>0$ and $a \in\left(0,\left(4 \phi^{-1}(T)\right)^{-1}\right]$. Then there exist constants $\left.C_{6.6}\right]_{i}=C_{6.6}\left(a, \beta, \chi, L_{0}, \phi, T, \lambda_{1}, \lambda_{2}\right)>0, i=1,2$ such that for any $x, y \in D$ with $\delta_{D}(x) \wedge \delta_{D}(y) \geqslant a \phi^{-1}(t),|x-y| \geqslant 1$, and $t \leqslant T$ we have

$$
p_{D}(t, x, y) \geqslant C\left[\sigma_{6.6}\right]_{1} t \exp \left\{-C_{[6.6}\left(|x-y|\left(\log \frac{T|x-y|}{t}\right)^{\frac{\beta-1}{\beta}} \wedge(|x-y|)^{\beta}\right)\right\} \text {. }
$$

Proof. Let $R_{1}:=|x-y|$. If either $1 \leqslant R_{1} \leqslant 2$ or $R_{1}\left(\log \left(T R_{1} / t\right)\right)^{(\beta-1) / \beta} \geqslant\left(R_{1}\right)^{\beta}$, the proposition holds by virtue of Proposition 6.4(2). Thus for the remainder of this proof we assume that $R_{1}>2$ and $R_{1}\left(\log \left(T R_{1} / t\right)\right)^{(\beta-1) / \beta}<\left(R_{1}\right)^{\beta}$, which is equivalent to $1<R_{1}\left(\log T R_{1} / t\right)^{-1 / \beta}$ and $R_{1} \exp \left(-R_{1}^{\beta}\right)<t / T$.

Let $k \geqslant 2$ be a positive integer such that

$$
R_{1}\left(\log \frac{T R_{1}}{t}\right)^{-1 / \beta} \leqslant k<R_{1}\left(\log \frac{T R_{1}}{t}\right)^{-1 / \beta}+1<2 R_{1}\left(\log \frac{T R_{1}}{t}\right)^{-1 / \beta}
$$

then $R_{1} / k \geqslant 2^{-1}\left(\log \left(T R_{1} / t\right)\right)^{1 / \beta} \geqslant 2^{-1}(\log 2)^{1 / \beta}=: c_{0}$.

By the assumption on $D$, there is a length parameterized curve $l \subset D$ connecting $x$ and $y$ such that $|l| \leqslant \lambda_{1} R_{1}$ and $\delta_{D}(l(u)) \geqslant \lambda_{2} a \phi^{-1}(t)$ for every $u \in[0,|l|]$. Let $r_{t}:=\left(2^{-1} \lambda_{2} a \phi^{-1}(t)\right) \wedge$ $\left(c_{0} / 2\right)$ and define $x_{i}:=l(i|l| / k)$ and $B_{i}:=B\left(x_{i}, r_{t}\right)$, with $i=0,1, \ldots, k$. For every $y_{i} \in B_{i}$, $\delta_{D}\left(y_{i}\right) \geqslant 2^{-1} \lambda_{2} a \phi^{-1}(t)>2^{-1} \lambda_{2} a \phi^{-1}(t / k)$ and

$$
\left|y_{i}-y_{i+1}\right| \leqslant\left|x_{i}-x_{i+1}\right|+2 r_{t} \leqslant \frac{|l|}{k}+c_{0} \leqslant\left(\lambda_{1}+1\right) \frac{R_{1}}{k} .
$$


By Proposition 6.4 (2) and (6.8), and using the facts that $t / k \leqslant T / 2$ and $R_{1} / k \geqslant c_{0}$, we have that for any $\left(y_{i}, y_{i+1}\right) \in B_{i} \times B_{i+1}$,

$p_{D}\left(t / k, y_{i}, y_{i+1}\right) \geqslant c_{1}\left(\frac{1}{\left[\phi^{-1}(t / k)\right]^{d}} \wedge \frac{t}{k} \cdot \nu\left(\left|y_{i}-y_{i+1}\right|\right) / \chi\left(\left|y_{i}-y_{i+1}\right|\right)\right) \geqslant c_{2} \frac{t}{k} \cdot \frac{e^{-c_{3}\left(R_{1} / k\right)^{\beta}}}{\phi\left(R_{1} / k\right)\left(R_{1} / k\right)^{d}}$

for some constants $c_{i}=c_{i}\left(a, L_{0}, \phi, \chi, \beta, T, \lambda_{1}\right)>0, i=2,3$. Since $\phi\left(R_{1} / k\right) \leqslant c_{4}\left(R_{1} / k\right)^{\bar{\alpha}}$ by (WS) with $R_{1} / k \geqslant c_{0}$, using (6.7), we have that

$$
\begin{aligned}
p_{D}\left(t / k, y_{i}, y_{i+1}\right) & \geqslant c_{2} \cdot c_{4} \frac{t}{T R_{1}}\left(\frac{k}{R_{1}}\right)^{\bar{\alpha}+d-1} e^{-c_{3}\left(R_{1} / k\right)^{\beta}} \\
& \geqslant c_{2} \cdot c_{4} \frac{t}{T R_{1}}\left(\log \frac{T R_{1}}{t}\right)^{-\frac{\bar{\alpha}+d-1}{\beta}}\left(\frac{t}{T R_{1}}\right)^{c_{3}} \geqslant c_{5}\left(\frac{t}{T R_{1}}\right)^{c_{6}}
\end{aligned}
$$

for some $c_{i}=c_{i}\left(a, L_{0}, \phi, \chi, \beta, T, \lambda_{1}\right), i=5,6$. Note that $r_{t} \geqslant c_{7}\left(t / T R_{1}\right)^{1 / \underline{\alpha}}$ for some $c_{7}=$ $c_{7}\left(a, \beta, \phi, \lambda_{2}\right)$ by (5.3) and the fact that $t / T R_{1} \leqslant 1 / 2$. Combining this with (6.9), (6.7) and by the semigroup property, we conclude that

$$
\begin{aligned}
p_{D}(t, x, y) & \geqslant \int_{B_{1}} \cdots \int_{B_{k-1}} p_{D}\left(t / k, x, y_{1}\right) \cdots p_{D}\left(t / k, y_{k-1}, y\right) d y_{1} \cdots d y_{k-1} \\
& \geqslant c_{8} \exp \left\{-c_{9} k \log \left(T R_{1} / t\right)\right\} \\
& \geqslant c_{8} \exp \left\{-c_{9}\left(2 R_{1} \log \left(\frac{T R_{1}}{t}\right)^{-1 / \beta}\right) \log \frac{T R_{1}}{t}\right\} \\
& \geqslant c_{8} \exp \left\{-2 c_{9} \cdot R_{1} \log \left(\frac{T R_{1}}{t}\right)^{1-1 / \beta}\right\} .
\end{aligned}
$$

Proofs of the lower bounds in Theorems 1.1 and 1.2. The lower bound of $p(t, x, y)$ in Theorem 1.1 follows from Proposition 6.4(1) with $D=\mathbb{R}^{d}$. The lower bound of $p(t, x, y)$ in Theorem 1.2 for the case $\beta \in[0,1]$ and the case $\beta \in(1, \infty]$ with $|x-y|<1$ follows from Proposition $6.4(2)$ with $D=\mathbb{R}^{d}$ and the remaining cases of Theorem 1.2 follows from Propositions 6.5 and 6.6 with $D=\mathbb{R}^{d}$.

\section{Lower bound estimates}

In this section, we first obtain the boundary decay in Lemma 7.4 using (4.8), Lemma 6.1 and Lemmas 7.1 and 7.2 below. Using the semigroup property, and then applying Lemma 7.4 and the preliminary lower bound estimates in Section 6, we will derive the upper bound estimate on $p_{D}(t, x, y)$ with the boundary decay terms for $t \leqslant T$ in $C^{1,1}$ open set $D$ with $C^{1,1}$ characteristics $\left(R_{0}, \Lambda\right)$. As before, we assume that $R_{0}<1$ and $\Lambda>1$.

We first introduce the next lemma (for the proof see [16, Lemma 3.3]).

Lemma 7.1. Suppose that $E \subset \mathbb{R}^{d}$ be an open set and $U_{1}, U_{2} \subset E$ be disjoint open subsets. If $x \in U_{1}, y \in U_{2}$ and $t>0$, we have

$$
p_{E}(t, x, y) \geqslant t \mathbb{P}_{x}\left(\tau_{U_{1}}>t\right) \mathbb{P}_{y}\left(\tau_{U_{2}}>t\right) \inf _{(u, w) \in U_{1} \times U_{2}} J(u, w) .
$$


For the remainder of the section, we assume that $Y$ is the symmetric pure jump Hunt process with the jumping intensity kernel $J$ satisfying the conditions $(\mathbf{J} 1.1),(\mathbf{J} 1.2)$ and $\left(\mathbf{K}_{\eta}\right)$. For any $T>0$, let

$$
\widehat{a}_{T}:=\widehat{a}_{T, R_{0}}:=\frac{R_{0}}{80 \phi^{-1}(T)},
$$

and for $x \in D$ we use $z_{x}$ to denote a point on $\partial D$ such that $\left|z_{x}-x\right|=\delta_{D}(x)$.

We first give the survival probability where $x$ is near the boundary of $D$ in the following lemma.

Lemma 7.2. Let $a \leqslant \widehat{a}_{T}$. Then, there exists a constant $C_{7.2}=G_{7.2}\left(a, \phi, L_{0}, L_{3}, \eta, \Lambda, T\right)>0$ such that for every $t \leqslant T$ and $x \in D$ with $\delta_{D}(x)<a \phi^{-1}(t)$ we have

$$
\mathbb{P}_{x}\left(\tau_{B\left(z_{x}, 10 a \phi^{-1}(t)\right) \cap D}>t / 3\right) \geqslant Q \overline{7.2} \frac{V\left(\delta_{D}(x)\right)}{\sqrt{t}} .
$$

Proof. Without loss of generality, we assume that $z_{x}=0$. Consider a coordinate system $C S:=C S_{0}$ such that $B\left(0, R_{0}\right) \cap D=\left\{y=\left(\widetilde{y}, y_{d}\right) \in B\left(0, R_{0}\right)\right.$ in $\left.C S: y_{d}>\varphi(\widetilde{y})\right\}$, where $\varphi$ is a $C^{1,1}$ function such that $\varphi(0)=0, \nabla \varphi(0)=(0, \ldots, 0),\|\nabla \varphi\|_{\infty} \leqslant \Lambda$, and $|\nabla \varphi(\widetilde{y})-\nabla \varphi(\widetilde{w})| \leqslant$ $\Lambda|\widetilde{y}-\widetilde{w}|$. Define $\varphi_{1}(\widetilde{y}):=2 \Lambda|\widetilde{y}|$ and $V:=\left\{y=\left(\widetilde{y}, y_{d}\right) \in B\left(0, R_{0}\right)\right.$ in $\left.C S: y_{d}>\varphi_{1}(\widetilde{y})\right\}$. Since $\varphi_{1}(\widetilde{y}) \geqslant 2 \Lambda|\widetilde{y}|^{2}$ for $y \in B\left(0, R_{0}\right)$, the mean value theorem yields $V \subset B\left(0, R_{0}\right) \cap D$.

Let $U_{1}:=B\left(0,2 a \phi^{-1}(t)\right) \cap D, U_{2}:=B\left(0,10 a \phi^{-1}(t)\right) \cap D$, and

$$
W:=\left\{y=\left(\widetilde{y}, y_{d}\right) \in B\left(0,8 a \phi^{-1}(t)\right) \backslash B\left(0,2 a \phi^{-1}(t)\right) \text { in } C S: y_{d}>\varphi_{1}(\widetilde{y})\right\} \subset V .
$$

Since $\Lambda|\widetilde{w}|=\varphi_{1}(\widetilde{w}) / 2<w_{d} / 2$ for $w \in W$, we have

$$
\delta_{D}(w)>\frac{\left(w_{d}-\varphi(\widetilde{w})\right)}{(1+\Lambda)}>\frac{\left(w_{d}-\Lambda|\widetilde{w}|\right)}{(1+\Lambda)}>\frac{w_{d}}{2(1+\Lambda)} \quad \text { for } w \in W .
$$

Moreover, since $|\widetilde{w}| \leqslant(2 \Lambda)^{-1}|w| \leqslant \Lambda^{-1} 4 a \phi^{-1}(t) \leqslant a \phi^{-1}(t)$ for $w \in W$, we have

$$
w_{d}^{2}=|w|^{2}-|\widetilde{w}|^{2} \geqslant\left(2 a \phi^{-1}(t)\right)^{2}-\left(a \phi^{-1}(t)\right)^{2} \geqslant\left(a \phi^{-1}(t)\right)^{2} \quad \text { for } w \in W .
$$

Combining (7.3) and (7.4), we obtain $\delta_{D}(w)>2^{-1}(1+\Lambda)^{-1} a \phi^{-1}(t)$ and $B\left(w, r_{1} a \phi^{-1}(t)\right) \subset U_{2}$ for $w \in W$, where $r_{1}:=(2(1+\Lambda))^{-1}$. By virtue of the strong Markov property, Lemma 6.1, and (4.8), we have

$$
\begin{aligned}
& \mathbb{P}_{x}\left(\tau_{U_{2}}>t / 3\right) \geqslant \mathbb{P}_{x}\left(\tau_{U_{2}}>t / 3, Y_{\tau_{U_{1}}} \in W\right)=\mathbb{E}_{x}\left[\mathbb{P}_{Y_{\tau_{U_{1}}}}\left(\tau_{U_{2}}>t / 3\right): Y_{\tau_{U_{1}}} \in W\right] \\
\geqslant & \mathbb{E}_{x}\left[\mathbb{P}_{Y_{\tau_{U_{1}}}}\left(\tau_{B\left(Y_{\tau_{U_{1}}}, r_{1} a \phi^{-1}(t)\right)}>t / 3\right): Y_{\tau_{U_{1}}} \in W\right] \geqslant\left(\inf _{z \in \mathbb{R}^{d}} \mathbb{P}_{z}\left(\tau_{B\left(z, r_{1} a \phi^{-1}(t)\right)}>t / 3\right)\right) \mathbb{P}_{x}\left(Y_{\tau_{U_{1}}} \in W\right) \\
\geqslant & C_{6.1} \mathbb{P}_{x}\left(Y_{\tau_{U_{1}}} \in W\right) \geqslant C_{6.1} \cdot C_{4.2} \frac{V\left(\delta_{D}(x)\right)}{V\left(8 a \phi^{-1}(t)\right)} \geqslant c_{1} \frac{V\left(\delta_{D}(x)\right)}{\sqrt{t}} .
\end{aligned}
$$

By the subadditivity of $V$ and (3.3),$V\left(8 a \phi^{-1}(t)\right) \leqslant(8 a+1) V\left(\phi^{-1}(t)\right) \asymp \sqrt{t}$, and therefore we obtain the last inequality.

We introduce the following definition for the subsequent lemma.

Definition 7.3. Let $0<\kappa_{1} \leqslant 1 / 2$. We say that an open set $D$ is $\kappa_{1}$-fat if there is $R_{1}>0$ such that for all $x \in \bar{D}$ and all $r \in\left(0, R_{1}\right]$ there is a ball $B\left(A_{r}(x), \kappa_{1} r\right) \subset D \cap B(x, r)$. The pair $\left(R_{1}, \kappa_{1}\right)$ are called the characteristics of the $\kappa_{1}$-fat open set $D$. 
Note that a $C^{1,1}$ open set $D$ with characteristics $\left(R_{0}, \Lambda\right)$ is a $\kappa_{1}$-fat set with characteristics $\left(R_{1}, \kappa_{1}\right)$ depending only on $R_{0}, \Lambda$, and $d$, and without loss of generality, we assume that $R_{0} \leqslant R_{1}$ (by choosing $R_{0}$ smaller if necessary). Let $A_{r}(x)$ is always the point $A_{r}(x) \in D$ in Definition 7.3 for $D$.

Recall that the function $\Psi$ is defined in (1.7).

Lemma 7.4. There exists a constant $C_{7.4}=C_{7.4}\left(\phi, L_{0}, L_{3}, \eta, R_{0}, \Lambda, T\right)>0$ such that, for every $t \leqslant T$ and $x \in D$, we can find $x_{1}$ with $\delta_{D}\left(x_{1}\right) \geqslant 2^{-1} \kappa_{1} \widehat{a}_{T} \phi^{-1}(t)$ and $\left|x_{1}-x\right| \leqslant 6 \widehat{a}_{T} \phi^{-1}(t)$ such that

$$
\int_{B\left(x_{1}, 4^{-1} \kappa_{1} \widehat{a}_{T} \phi^{-1}(t)\right)} p_{D}(t / 3, x, z) d z \geqslant C_{7.4} \Psi(t, x) .
$$

Proof. Let $r_{t}:=\widehat{a}_{T} \phi^{-1}(t) \leqslant R_{0} / 80 \leqslant 1 / 80$ and we consider the case $\delta_{D}(x)<2^{-1} \kappa_{1} r_{t}$ first. In this case we let $x_{1}:=A_{6 r_{t}}\left(z_{x}\right)$ and denote $B_{x_{1}}:=B\left(x_{1}, 4^{-1} \kappa_{1} r_{t}\right)$ and $B_{z_{x}}:=B\left(z_{x}, 5 \kappa_{1} r_{t}\right) \cap D$ so that $B_{x_{1}} \cap B_{z_{x}}=\emptyset$. For any $u \in B_{z_{x}}$ and $w \in B_{x_{1}}$,

$$
|u-w| \leqslant\left|u-z_{x}\right|+\left|z_{x}-x_{1}\right|+\left|x_{1}-w\right| \leqslant 12 \kappa_{1} r_{t} \leqslant 1 .
$$

Since $\phi\left(12 \kappa_{1} r_{t}\right) \asymp \phi\left(\phi^{-1}(t)\right)=t$ by (WS), using (J1.1), (1.2) and (1.4), we have that

$$
\inf _{(u, w) \in B_{z_{x}} \times B_{x_{1}}} J(u, w) \geqslant L_{0}^{-1} \phi\left(12 \kappa_{1} r_{t}\right)^{-1}\left|12 \kappa_{1} r_{t}\right|^{-d} \geqslant c_{1} t^{-1}\left[\phi^{-1}(t)\right]^{-d}
$$

for some constant $c_{1}:=c_{1}\left(\phi, L_{0}, R_{0}, \Lambda, T\right)>0$. Therefore, Lemmas 7.1, 7.2, and 6.1 implies that

$$
\begin{aligned}
\int_{B_{x_{1}}} p_{D}(t / 3, x, z) d z & \geqslant \frac{t}{3} \int_{B_{x_{1}}} \mathbb{P}_{x}\left(\tau_{B_{z_{x}}}>t / 3\right) \mathbb{P}_{z}\left(\tau_{B_{x_{1}}}>t / 3\right) \cdot \inf _{(u, w) \in B_{z_{x}} \times B_{x_{1}}} J(u, w) d z \\
& \geqslant \frac{1}{3} \mathbb{P}_{x}\left(\tau_{B_{z_{x}}}>t / 3\right) \cdot C_{6.1} \int_{B_{x_{1}}} d z \cdot c_{1} \frac{1}{\left[\phi^{-1}(t)\right]^{d}} \\
& =c_{2} \mathbb{P}_{x}\left(\tau_{B_{z_{x}}}>t / 3\right) \geqslant c_{2} \cdot C_{7.2} \frac{V\left(\delta_{D}(x)\right)}{\sqrt{t}} .
\end{aligned}
$$

For $\delta_{D}(x) \geqslant 2^{-1} \kappa_{1} r_{t}$, let $x_{1}=x$ and $B_{x_{1}}:=B\left(x_{1}, 4^{-1} \kappa_{1} r_{t}\right)$. By Lemma 6.1,

$$
\int_{B_{x_{1}}} p_{D}(t / 3, x, z) d z \geqslant \int_{B_{x_{1}}} p_{B_{x_{1}}}(t / 3, x, z) d z=\mathbb{P}_{x}\left(\tau_{B_{x_{1}}}>t / 3\right)>C 6.1
$$

and this proves the lemma.

We are now ready to give the proof of the lower bound estimates for $p_{D}(t, x, y)$. Recall our assumption that $D$ is a $C^{1,1}$ open set. When the jumping intensity $J$ of $Y$ satisfies (J2), for the cases $\beta \in(1, \infty)$ with $|x-y| \geqslant 1$ and $\beta=\infty$ with $|x-y| \geqslant 4 / 5$, we assume in addition that the path distance in each connected component of $D$ is comparable to the Euclidean distance with characteristic $\lambda_{1}$. Note that combining this assumption with $C^{1,1}$ assumption entails that $D$ satisfies the assumption made before Proposition 6.5.

Proofs of the lower bound of $p_{D}(t, x, y)$ in Theorems 1.3(1), 1.5)(2) and 1.5)(3). Let $r_{t}:=\widehat{a}_{T} \phi^{-1}(t) \leqslant R_{0} / 80 \leqslant 1 / 80$. By Lemma 7.4 , for any $x, y \in D$, there exists $x_{1}, y_{1} \in D$ such that $\delta_{D}\left(x_{1}\right) \wedge \delta_{D}\left(y_{1}\right) \geqslant 2^{-1} \kappa_{1} r_{t}$ and $\left|x_{1}-x\right| \vee\left|y_{1}-y\right| \leqslant 6 r_{t}$, and

$$
\int_{B_{x_{1}}} p_{D}(t / 3, x, z) d z \int_{B_{y_{1}}} p_{D}(t / 3, y, z) d z \geqslant C_{[7.4}^{2} \Psi(t, x) \Psi(t, y),
$$


where $B_{x_{1}}:=B\left(x_{1}, 4^{-1} \kappa_{1} r_{t}\right)$ and $B_{y_{1}}:=B\left(y_{1}, 4^{-1} \kappa_{1} r_{t}\right)$. Thus by the semigroup property,

$$
\begin{aligned}
p_{D}(t, x, y) & =\int_{D} \int_{D} p_{D}(t / 3, x, u) p_{D}(t / 3, u, w) p_{D}(t / 3, w, y) d u d w \\
& \geqslant \int_{B_{x_{1}}} p_{D}(t / 3, x, u) d u \int_{B_{y_{1}}} p_{D}(t / 3, y, w) d w\left(\inf _{(u, w) \in B_{x_{1}} \times B_{y_{1}}} p_{D}(t / 3, u, w)\right) \\
& \geqslant G_{7.4}^{2} \Psi(t, x) \Psi(t, y) \inf _{(u, w) \in B_{x_{1}} \times B_{y_{1}}} p_{D}(t / 3, u, w) .
\end{aligned}
$$

We now carefully calculate the lower bounds of $p_{D}(t / 3, u, w)$ on $B_{x_{1}} \times B_{y_{1}}$. Since $\left|x-x_{1}\right| \vee$ $\left|y-y_{1}\right| \leqslant 6 r_{t}$, for $u \in B_{x_{1}}$ and $w \in B_{y_{1}}$ we have

$$
\begin{aligned}
& |x-y|-6^{-1} \leqslant|x-y|-\left(12+\left(\kappa_{1} / 2\right)\right) r_{t} \\
& \leqslant|u-w| \leqslant|x-y|+\left(12+\left(\kappa_{1} / 2\right)\right) r_{t} \leqslant|x-y|+6^{-1}
\end{aligned}
$$

and $\delta_{D}(u) \wedge \delta_{D}(w) \geqslant 4^{-1} \kappa_{1} r_{t}$.

We first assume that the jumping intensity kernel $J$ satisfies the condition (J2). Let $\beta \in$ $[0,1]$. If $|x-y| \leqslant 15 r_{t}$, then $|u-w| \leqslant 28 r_{t}<1$ and $\phi(|u-w|)|u-w|^{d} \leqslant c_{1} t\left[\phi^{-1}(t)\right]^{d}$ since $\phi(|u-w|) \leqslant \phi\left(28 \kappa_{1} r_{t}\right) \asymp \phi\left(\phi^{-1}(t)\right)=t$ by (WS). If $|x-y|>15 r_{t}$, then $|u-w| \leqslant|x-y|+6^{-1}$ and $\phi(|u-w|)|u-w|^{d} \leqslant c_{2} \phi(|x-y|)|x-y|^{d}$ since $r \rightarrow \phi(r)$ is increasing and using (WS). Combining these observations with Proposition 6.4(2), (1.2) and (1.5),

$p_{D}(t / 3, u, w) \geqslant c_{3}\left(\left[\phi^{-1}(t)\right]^{-d} \wedge t e^{-\gamma_{2}|u-w|^{\beta}} \nu(|u-w|)\right) \geqslant c_{4}\left(\left[\phi^{-1}(t)\right]^{-d} \wedge t e^{-\gamma_{2}|x-y|^{\beta}} \nu(|x-y|)\right)$.

If $\beta \in(1, \infty]$ and $|x-y| \leqslant 4 / 5$, then (7.8) yields $|u-w| \leqslant|x-y|+6^{-1}<1$. Similar to the above case, considering the cases $|x-y| \leqslant 15 r_{t}$ and $|x-y|>15 r_{t}$ separately, we have $p_{D}(t / 3, u, w) \geqslant c_{5}\left(\left[\phi^{-1}(t)\right]^{-d} \wedge t \cdot \nu(|x-y|)\right)$. Moreover,

(1) if $\beta \in(1, \infty)$ and $4 / 5 \leqslant|x-y|<2$, then $|u-w| \asymp 1$. Thus by Proposition 6.4(2), we have $p_{D}(t / 3, u, w) \geqslant c_{6} t$.

Hence combining (7.7) with these observations, we have proved the lower bound of $p_{D}(t, x, y)$ in Theorem 1.5)(2).

Suppose the jumping intensity kernel $J$ satisfies the condition (J1) and $M>0$. Let $|x-y|<M$. Similar to the $\beta \in[0,1]$ case, applying Proposition 6.4)(1) instead of Proposition 6.4(2) and considering $|x-y| \leqslant 15 r_{t} \wedge M$ and $15 r_{t} \wedge M<|x-y| \leqslant M$ separately, we have $p_{D}(t / 3, u, w) \geqslant c_{7}\left(\left[\phi^{-1}(t)\right]^{-d} \wedge t \cdot \nu(|x-y|)\right)$. Hence combining (7.7) with this, we have proved the lower bound of $p_{D}(t, x, y)$ in Theorem [1.3)(1).

We now return to the assumption that the jumping intensity kernel $J$ satisfies the condition (J2), further assume that the path distance in $D$ is comparable to the Euclidean distance. If $4 / 5 \leqslant|x-y|$, then (7.8) yields $|u-w| \asymp|x-y|$. Recall that we have already discuss the case $\beta \in(1, \infty)$ and $4 / 5 \leqslant|x-y|<2$ in (1). We now consider $p_{D}(t / 3, u, w)$ in each of the remaining cases.

(2) If $\beta=\infty$ and $4 / 5 \leqslant|x-y|<2$, then by Propositions 6.4 and 6.5 , we have

$$
p_{D}(t / 3, u, w) \geqslant c_{8} \frac{4 t}{5 T|x-y|} \geqslant c_{8}\left(\frac{4 t}{5 T|x-y|}\right)^{5|x-y| / 4} .
$$


(3) If $\beta \in(1, \infty)$ and $2 \leqslant|x-y|$, then $1<|u-w|$ and from Proposition 6.6 and (7.8) we obtain

$$
\begin{aligned}
& p_{D}(t / 3, u, w) \geqslant c_{9} t \exp \left\{-c_{10}\left(|u-w|\left(\log \frac{T|u-w|}{t}\right)^{\frac{\beta-1}{\beta}} \wedge|u-w|^{\beta}\right)\right\} \\
& \geqslant c_{9} t \exp \left\{-c_{10}\left((5|x-y| / 4)\left(\log \left(\frac{T\left(|x-y|+6^{-1}\right)}{t}\right)\right)^{\frac{\beta-1}{\beta}} \wedge(5|x-y| / 4)^{\beta}\right)\right\} \\
& \geqslant c_{9} t \exp \left\{-c_{11}\left(|x-y|\left(\log \frac{T|x-y|}{t}\right)^{\frac{\beta-1}{\beta}} \wedge|x-y|^{\beta}\right)\right\} .
\end{aligned}
$$

The last inequality comes from the inequality $\log r \leqslant \log (r+b) \leqslant 2 \log r$ for $r \geqslant 2 \vee b>0$.

(4) If $\beta=\infty$ and $2 \leqslant|x-y|$, then $1<|u-w|$ and from Proposition 6.5 and (7.8) we have

$$
\begin{aligned}
& p_{D}(t / 3, u, w) \geqslant c_{12}\left(\frac{t}{T|u-w|}\right)^{c_{13}|u-w|} \geqslant c_{12}\left(\frac{t}{T\left(|x-y|+6^{-1}\right)}\right)^{c_{13} 5|x-y| / 4} \\
& \geqslant c_{12}\left(\frac{t}{T|x-y|}\right)^{c_{13} 5|x-y| / 2} \geqslant c_{12}\left(\frac{4 t}{5 T|x-y|}\right)^{c_{13} 5|x-y| / 2} .
\end{aligned}
$$

The second last inequality holds by virtue of the inequality $r^{2} \geqslant r+b$ for $r \geqslant 2 \vee b>0$.

Hence combining (7.7) with the above observations $(1)-(4)$ on the lower bound of $p_{D}(t / 3, u, w)$, we have proved the lower bound of $p_{D}(t, x, y)$ in Theorem 1.5)(3).

Proof of Theorem 1.5(4). Let $D(x)$ and $D(y)$ be connected components containing $x$ and $y$, respectively. By definition of a $C^{1,1}$ open set, the distance between $x$ and $y$ is at least $R_{0}$. Using Lemma 7.4, we find that $x_{1} \in D(x)$ and $y_{1} \in D(y)$. Define $B_{x_{1}}$ and $B_{y_{1}}$ in the same way as when beginning the proof of Theorem 1.5)(2) and 1.5) (3) so that (7.6) holds.

For any $u \in B_{x_{1}}$ and $w \in B_{y_{1}}$, since $3 R_{0} / 4 \leqslant 3|x-y| / 4 \leqslant|u-w| \leqslant 5|x-y| / 4$, by Proposition $6.4(2)$ and (5.3),

$$
p_{D}(t / 3, u, w) \geqslant c_{1} t \nu(|u-w|) e^{-\gamma_{2}|u-w|^{\beta}} \geqslant c_{2} t \nu(|x-y|) e^{-\gamma_{2}(5|x-y| / 4)^{\beta}} .
$$

By the semigroup property, combining (7.6) and this observation, we conclude that

$$
\begin{aligned}
p_{D}(t, x, y) & \geqslant \int_{B_{x_{1}}} \int_{B_{y_{1}}} p_{D}(t / 3, x, w) p_{D}(t / 3, u, w) p_{D}(t / 3, w, y) d w d v \\
& \geqslant \int_{B_{x_{1}}} p_{D}(t / 3, x, u) d u \int_{B_{y_{1}}} p_{D}(t / 3, y, w) d w \cdot \inf _{(u, w) \in B_{x_{1}} \times B_{y_{1}}} p_{D}(t / 3, u, w) \\
& \geqslant c_{3} \Psi(t, x) \Psi(t, y) \cdot t \nu(|x-y|) e^{-\gamma_{2}(5|x-y| / 4)^{\beta}} .
\end{aligned}
$$

Proofs of Theorems 1.3(2) and 1.5(5). Using Lemmas 5.2 and 7.2 instead of [17, (5.1) and (5.10)], and by the fact that (3.3) and $D$ is bounded (and connected when $J$ satisfies the condition (J2) and $\beta=\infty)$, we can obtain the large time heat kernel estimates for $p_{D}(t, x, y)$ following the proofs of [17, Theorems 1.3(iii) and 1.5(iii)], so we omit the proofs. 


\section{Green function and boundary Harnack inequality}

In this section we give the Green function estimates and establish the boundary Harnack inequality as applications of the Dirichlet heat kernel estimates.

Proof of Theorem 1.6. When $d \geqslant 2$, the proof of Green function estimates is almost identical to the one of [17, Section 7]. Thus we skip the proof.

Suppose $d=1$. Note that by the inequality in Proposition 3.1, we have

$$
V^{\prime}(r) \leqslant c \frac{V(r)}{r} \quad \text { for } 0<r \leqslant M,
$$

Using (8.1) instead of [17, (7.3)], one can obtain the Green function estimates by following the proofs in [17, Section 7] line by line. Indeed, for any $T>0$, let

$$
K_{T}(a, r):=a+\phi(r) \int_{\phi(r) / T}^{1}\left(1 \wedge \frac{u a}{\phi(r)}\right) \frac{1}{u^{2} \phi^{-1}\left(u^{-1} \phi(r)\right)} d u+\frac{\phi(r)}{r}\left(1 \wedge \frac{a}{\phi(r)}\right)
$$

which is defined in [17, (7.4)]. By the same proof of [17, Theorem 7.3(iii)], we have that

$$
G_{D}(x, y) \asymp K_{T_{1}}(a(x, y),|x-y|)
$$

where $a(x, y)=\sqrt{\phi\left(\delta_{D}(x)\right)} \sqrt{\phi\left(\delta_{D}(y)\right)}$. Recall that $C_{I}$ is the constant in (15.3). Let $T_{1}:=$ $\left(2 \vee\left(2 C_{I}\right)^{\bar{\alpha}}\right) \phi(\operatorname{diam}(D))$. Since $0<a(x, y) \leqslant \phi(\operatorname{diam}(D))=\left(2^{-1} \wedge\left(2 C_{I}\right)^{-\bar{\alpha}}\right) T_{1}$ and $\phi(|x-y|) \leqslant$ $\phi(\operatorname{diam}(D)) \leqslant T_{1} / 2$, it is enough to show that for any $T>0$ and for any $0<a \leqslant\left(2^{-1} \wedge\right.$ $\left.\left(2 C_{I}\right)^{-\bar{\alpha}}\right) T$ and $0<\phi(r) \leqslant T / 2$,

$$
K_{T}(a, r) \asymp \frac{a}{r} \wedge\left(\frac{a}{\phi^{-1}(a)}+\left(\int_{r}^{\phi^{-1}(a)} \frac{\phi(s)}{s^{2}}\right)^{+}\right)
$$

where $x^{+}:=x \vee 0$.

When $0<a<\phi(r) \leqslant T / 2$, the proof of (8.2) is the same as that of [17, Lemma 7.2]. Now we assume that $\phi(r) \leqslant a \leqslant\left(2^{-1} \wedge\left(2 C_{I}\right)^{-\bar{\alpha}}\right) T$. Using (3.3), we have $c_{1}^{-1} V(r)^{2} \leqslant \phi(r) \leqslant c_{1} V(r)^{2}$ for some constant $c_{1}>1$. Thus by the change of variable $u=V(r)^{2} / V(s)^{2}$, we have that

$$
\int_{\phi(r) / T}^{\phi(r) / a} \frac{a d u}{u \phi^{-1}\left(u^{-1} \phi(r)\right)} \leqslant \int_{V(r)^{2} /\left(c_{1} T\right)}^{c_{1} V(r)^{2} / a} \frac{a d u}{u \phi^{-1}\left(u^{-1} \phi(r)\right)} \leqslant \int_{1 / V\left(a / c_{1}\right)^{2}}^{1 / V\left(c_{1} T\right)^{2}} \frac{2 a}{\phi^{-1}\left(c_{1}^{-1} V(s)^{2}\right)} \frac{V^{\prime}(s)}{V(s)} d s .
$$

Since $\phi^{-1}\left(c_{1}^{-1} V(s)^{2}\right) \geqslant \phi^{-1}\left(c_{1}^{-2} \phi(s)\right) \geqslant c_{2} s$ by (3.3) and (5.3), combining this with (8.1), we have that

$$
\int_{\phi(r) / T}^{\phi(r) / a} \frac{a \cdot d u}{u \phi^{-1}\left(u^{-1} \phi(r)\right)} \leqslant c_{3} a \int_{1 / V\left(a / c_{1}\right)^{2}}^{1 / V\left(c_{1} T\right)^{2}} \frac{1}{s^{2}} d s \leqslant c_{4} \frac{a}{\phi^{-1}(a)} .
$$

For the last inequality, we again used (3.3) and (5.3). Applying (8.3) to the proof of the upper bound for $K_{T}(a, r)$ in [17, (7.6)], and following the rest of the proof of [17, Theorem 7.3(iii)] for the $\phi(r) \leqslant a \leqslant\left(2^{-1} \wedge\left(2 C_{I}\right)^{-\bar{\alpha}}\right) T$ case, we obtain (8.2) and hence we prove Theorem 1.6 for all dimension.

To prove Theorem 1.8 we use the above estimates of Green function and the following the scale and translate invariant boundary Harnack inequality. 
Proposition 8.1. Suppose that $D$ is an open set in $\mathbb{R}^{d}$. Let $Y$ be a symmetric pure jump Hunt process whose jumping intensity kernel $J$ satisfies the conditions $(\mathbf{J} \mathbf{1}),(\mathbf{L}),(\mathbf{C})$ and $\left(\mathbf{K}_{\eta}\right)$. Then, there exists $c=c\left(\phi, \eta, L_{0}, L_{3}, d\right)$ such that for any $0<r<1, z \in \partial D$ and any nonnegative functions $f, g$ in $\mathbb{R}^{d}$ which are regular harmonic in $D \cap B(z, r)$ with respect to $Y$, and vanish in $D^{c} \cap B(z, r)$, we have

$$
\frac{f(x)}{f(y)} \leqslant c \frac{g(x)}{g(y)} \quad \text { for any } x, y \in D \cap B(z, 2 r / 3) .
$$

Proof. To prove the claim we use [7]. We only have to check assumptions stated therein. Note that $(\mathrm{C})$ is an uniform version of [7, Assumption C]. Thus there is a constant $c_{(2.7)}$ in [7] satisfies $c_{(2.7)}\left(x_{0}, R_{1}, R_{2}\right)=C^{*}\left(\phi, d, R_{1} / R_{2}\right)$ for any $x_{0} \in \mathbb{R}^{d}$ and $0<R_{1}<R_{2} \leqslant 2$. Let $0<r<1$ and $2 / 3<a \leqslant 2$.

We first check the bounds on the constants $c_{(2.8)}$ and $c_{(2.9)}$ in [7]. In our case, the constants $c_{(2.8)}$ and $c_{(2.9)}$ in [7] can be taken as

$$
c_{(2.8)}\left(x_{0}, a r, 2 r\right):=\inf _{\left\{a r \leqslant\left|x_{0}-y\right| \leqslant 2 r\right\}} J\left(x_{0}, y\right) \quad \text { and } \quad c_{(2.9)}\left(x_{0}, r\right) \leqslant C^{*}\left(\int_{\mathbb{R}^{d} \backslash B\left(x_{0}, 2 r\right)} J\left(x_{0}, y\right) d y\right)^{-1}
$$

where $C^{*}=C^{*}(\phi, d, 1 / 2)$ is the constant in (C). (see [7, (2.8) and (2.9)] and the last display of [7, Proposition 2.9]). Then by (J1.3) and (WS) for $c_{(2.8)}$, and by $(\mathbf{J} 1.1),(\mathbf{J} 1.2)(1.4)$ and (WS) for $c_{(2.9)}$, we have that

$$
c_{(2.8)}\left(x_{0}, a r, 2 r\right) \geqslant c_{1} \phi(r)^{-1} r^{-d} \quad \text { and } \quad c_{(2.9)}\left(x_{0}, r\right) \leqslant c_{2} \phi(r)
$$

where the constant $c_{1}>0$ depends on $\phi, a$ and $d$, and the constant $c_{2}>0$ depends on $\phi, L_{0}$ and $d$.

We now check [7, Assumption A-D] (and its scale and translate invariant version) holds. First of all, since $p(t, x, y)$ is continuous, clearly the transition operators $T_{t}$ of $Y$ is strong Feller. Recall that we assume that $T_{t}$ is Feller, that is, $T_{t}$ maps $C_{0}\left(\mathbb{R}^{d}\right)$ into $C_{0}\left(\mathbb{R}^{d}\right)$. Since $Y$ is symmetric, [7, Assumption A] holds.

Let $\widehat{A}$ be the corresponding generator on $C_{0}\left(\mathbb{R}^{d}\right)$ defined as

$$
\begin{aligned}
\widehat{A} u & :=\lim _{t \rightarrow 0} \frac{T_{t} u-u}{t} \quad \text { (strong limit) and } \\
D(\widehat{A}) & :=\left\{u \in C_{0}\left(\mathbb{R}^{d}\right): \widehat{A} u<\infty\right\} .
\end{aligned}
$$

Recall the operator $\mathcal{L} g(x)=P . V . \int(g(y)-g(x)) J(x, y) d y$ defined in (2.1). Then

$$
C_{c}^{2}\left(\mathbb{R}^{d}\right) \subset D(\widehat{A}) \quad \text { and } \quad \widehat{A} u=\mathcal{L} u \quad \text { for any } u \in C_{c}^{2}\left(\mathbb{R}^{d}\right) .
$$

Indeed, we first obtain that for any $u \in C_{c}^{2}\left(\mathbb{R}^{d}\right), \mathcal{L} u \in C_{0}\left(\mathbb{R}^{d}\right)$ by $(\mathbf{L})$ and so,

$$
\left\|T_{t}(\mathcal{L} u)-\mathcal{L} u\right\|_{\infty} \rightarrow 0 \quad \text { as } t \rightarrow 0 .
$$

Since, from Lemma 2.2, $M_{t}^{u}=u\left(Y_{t}\right)-u\left(Y_{0}\right)-\int_{0}^{t} \mathcal{L} u\left(Y_{s}\right) d s$ is $\mathbb{P}_{x}$-martingale with respect to the filtration of $Y$, we have that

$$
\frac{T_{t} u(x)-u(x)}{t}=\frac{1}{t} \mathbb{E}_{x}\left[\int_{0}^{t} \mathcal{L} u\left(Y_{s}\right) d s\right]
$$


Thus we obtain that for any $u \in C_{c}^{2}\left(\mathbb{R}^{d}\right)$,

$$
\sup _{x}\left|\frac{T_{t} u(x)-u(x)}{t}-\mathcal{L} u(x)\right|=\sup _{x}\left|\frac{1}{t} \int_{0}^{t} T_{s} \mathcal{L} u(x)-\mathcal{L} u(x) d s\right| \leqslant \frac{1}{t} \int_{0}^{t}\left\|T_{t}(\mathcal{L} u)-\mathcal{L} u\right\|_{\infty} d s,
$$

and combining this with (8.6), we conclude (8.5). Therefore, [7, Assumption B] holds with $\mathcal{D}=C_{c}^{2}\left(\mathbb{R}^{d}\right)$.

For $0<R_{1}<R_{2}$, let $A\left(x, R_{1}, R_{2}\right)=\left\{y \in \mathbb{R}^{d}: R_{1}<|x-y|<R_{2}\right\}$ be the open annulus around $x$, and $\bar{A}\left(x, R_{1}, R_{2}\right)$ the closure of $A\left(x, R_{1}, R_{2}\right)$. For every compact set $K$ and open set $U$ satisfying $K \subset U \subset \mathbb{R}^{d}$, let

$$
\mathcal{F}_{K, U}:=\left\{f \in C_{c}^{2}\left(\mathbb{R}^{d}\right): f \equiv 1 \text { in } K, f \equiv 0 \text { in } U^{c}, \text { and } 0 \leqslant f(x) \leqslant 1\right\},
$$

and $\varrho(K, U):=\inf _{f \in \mathcal{F}_{K, U}} \sup _{x} \mathcal{L} f(x)$. Then by Lemma 2.1 and (WS), for $2 / 3<a<b \leqslant 1$ there exist $c_{3}=c_{3}\left(\phi, \eta, L_{0}, L_{3}, a, b\right)$ such that for any $x_{0} \in \mathbb{R}^{d}$ and $0<r<1$,

$$
\widehat{\varrho}\left(x_{0}, a r, b r\right):=\varrho\left(\bar{A}\left(x_{0}, a r, b r\right), A\left(x_{0}, 2 r / 3,2 r\right)\right)+\varrho\left(\bar{B}\left(x_{0}, a r\right), B\left(x_{0}, b r\right)\right) \leqslant c_{3} \phi(r)^{-1} .
$$

Let $B_{u}:=B\left(x_{0}, u\right)$ be a ball centered at $x_{0}$ with radius $u>0$. Let $d \geqslant 1,0<r<1$ and $x, y \in B_{r}$. By Theorem 1.1 (with $M=2$ and $T=\phi(2)$ ) and the semigroup property we have, for $t_{0}=\phi(|x-y|)$

$$
\begin{aligned}
G_{B_{r}}(x, y) & \leqslant \int_{0}^{t_{0}} p(s, x, y) d s+\int_{0}^{\infty} p_{B_{r}}\left(s+t_{0}, x, y\right) d s \\
& \leqslant q_{1.1} \int_{0}^{t_{0}} s \nu(|x-y|) d s+\int_{B_{r}} p\left(t_{0}, z, y\right) G_{B_{r}}(x, z) d z \\
& \leqslant q_{1.1}\left(t_{0}^{2} \nu(|x-y|)+\left[\phi^{-1}\left(t_{0}\right)\right]^{-d} \mathbb{E}_{x} \tau_{B_{r}}\right) \\
& =\frac{G[1.1}{|x-y|^{d}}\left(\phi(|x-y|)+\mathbb{E}_{x} \tau_{B_{r}}\right) .
\end{aligned}
$$

Let $5 / 6<a<1$. For $x \in B_{5 r / 6}$ and $y \in B_{r} \backslash B_{a r},(a-5 / 6) r \leqslant|x-y| \leqslant 2 r$. Hence, by (8.4) and (WS) we obtain

$$
c_{(2.10)}\left(x_{0}, 5 r / 6, a r, r\right):=\sup _{\substack{x \in B_{5 r / 6} \\ y \in B_{r} \backslash B_{a r}}} G_{B_{r}}(x, y) \leqslant c_{4} \frac{\phi(r)}{r^{d}} .
$$

where the constant $c_{4}$ depends on $\phi, a$ and $d$. Hence [7, Assumption D] holds.

We have observed that [7, Assumption A-Assumption D] hold. In addition, by (8.4), (8.7) and (8.9), the upper bound of the constants $c_{(3.9)}, c_{(3.11)}$ and $c_{(1.1)}$ in [7] from the expressions of the constants $c_{(3.9)}, c_{(3.11)}$ and $c_{(1.1)}$ in [7, (3.9)-(3.11)] so that for any $x_{0} \in \mathbb{R}^{d}$ and $0<r<1$,

$$
\begin{aligned}
& c_{(3.9)}\left(x_{0}, 5 r / 6,11 r / 12, r\right) \leqslant c_{6} \frac{\phi(r)}{r^{d}}, \\
& c_{(3.11)}\left(x_{0}, 5 r / 6, r\right) \leqslant 2 c_{(3.9)}\left(x_{0}, 5 r / 6,11 r / 12, r\right) \\
& \quad \cdot \max \left(\frac{\widehat{\varrho}\left(x_{0}, 11 r / 12, r\right)}{c_{(2.8)}\left(x_{0}, 11 r / 12,2 r\right)},|B(0,1)| C^{*}(\phi, d, 1 / 2) r^{d}\right) \leqslant c_{7} \phi(r), \quad \text { and } \\
& c_{(1.1)}\left(x_{0}, 2 r / 3, r\right) \leqslant\left(\widehat{\varrho}\left(x_{0}, 3 r / 4,5 r / 6\right) \cdot c_{(3.11)}\left(x_{0}, 5 r / 6, r\right)+C^{*}(\phi, d, 9 / 10)\right)^{4} \leqslant c_{8}
\end{aligned}
$$

where the constants $c_{i}, i=6,7,8$ are depending only on $\phi, \eta, L_{0}, L_{3}$ and $d$. Therefore, we obtain the scaling and translation invariant version of [7, (BHI)] for $r<1$, with the constant $c_{(1.1)}=c_{(1.1)}\left(x_{0}, 2 r / 3, r\right)$ which is independent of $r<1$ and $x_{0} \in \mathbb{R}^{d}$. 
Alternatively, one can check the conditions in [35, Section 4], which also provides [35, Corollary 4.2], the scaling and translation invariant version of (BHI).

We now use the above proposition to prove Theorem 1.8 .

Proof of Theorem 1.8. Suppose that $D$ is a $C^{1,1}$ open set in $\mathbb{R}^{d}$ with characteristics $\left(R_{0}, \Lambda\right)$. Since $D$ is a $C^{1,1}$ open set, it is easy to see that for any $z \in \partial D$ there exits a bounded $C^{1,1}$ open set $U$ in $\mathbb{R}^{d}$ whose characteristics depend only on $R_{0}$ and $\Lambda$ (independent of $z \in \partial D$ ) such that $B\left(z, 7 R_{0} / 8\right) \cap D \subset U \subset B\left(z, R_{0}\right) \cap D$ (if $d=1$ we can take $U=\left(z, z+R_{0}\right)$ or $U=\left(z-R_{0}, z\right)$ ). Choose a point $z_{0} \in U \backslash \overline{B\left(z, 3 R_{0} / 4\right)}$ and let $g_{1}(x)=G_{U}\left(x, z_{0}\right)$. Since $g_{1}$ is regular harmonic in $D \cap B\left(z, 3 R_{0} / 4\right)$, applying Proposition 8.1 we obtain

$$
\frac{f(x)}{f(y)} \leqslant c_{1} \frac{g_{1}(x)}{g_{1}(y)}, \quad x, y \in D \cap B(z, r / 2) .
$$

Theorem [1.6 implies the claim of the theorem.

\section{References}

[1] M. T. Barlow, R. F. Bass, Z.-Q. Chen, and M. Kassmann. Non-local Dirichlet forms and symmetric jump processes. Trans. Amer. Math. Soc., 361(4):1963-1999, 2009.

[2] J. Bertoin. Lévy processes, volume 121 of Cambridge Tracts in Mathematics. Cambridge University Press, Cambridge, 1996.

[3] K. Bogdan, T. Grzywny, and M. Ryznar. Heat kernel estimates for the fractional Laplacian with Dirichlet conditions. Ann. Probab., 38(5):1901-1923, 2010.

[4] K. Bogdan, T. Grzywny, and M. Ryznar. Density and tails of unimodal convolution semigroups. J. Funct. Anal., 266(6):3543-3571, 2014.

[5] K. Bogdan, T. Grzywny, and M. Ryznar. Dirichlet heat kernel for unimodal Lévy processes. Stochastic Process. Appl., 124(11):3612-3650, 2014.

[6] K. Bogdan, T. Grzywny, and M. Ryznar. Barriers, exit time and survival probability for unimodal Lévy processes. Probab. Theory Related Fields, 162(1-2):155-198, 2015.

[7] K. Bogdan, T. Kumagai, and M. Kwaśnicki. Boundary Harnack inequality for Markov processes with jumps. Trans. Amer. Math. Soc., 367(1):477-517, 2015.

[8] L. A. Caffarelli and A. Vasseur. Drift diffusion equations with fractional diffusion and the quasi-geostrophic equation. Ann. of Math. (2), 171(3):1903-1930, 2010.

[9] Z.-Q. Chen. On notions of harmonicity. Proc. Amer. Math. Soc., 137(10):3497-3510, 2009.

[10] Z.-Q. Chen, P. Kim, and T. Kumagai. Weighted poincaré inequality and heat kernel estimates for finite range jump processes. Math. Ann., 342(4):833-883, 2008.

[11] Z.-Q. Chen, P. Kim, and T. Kumagai. On heat kernel estimates and parabolic Harnack inequality for jump processes on metric measure spaces. Acta Math. Sin. (Engl. Ser.), 25(7):1067-1086, 2009. 
[12] Z.-Q. Chen, P. Kim, and T. Kumagai. Global heat kernel estimates for symmetric jump processes. Trans. Amer. Math. Soc., 363(9):5021-5055, 2011.

[13] Z.-Q. Chen, P. Kim, and R. Song. Heat kernel estimates for the Dirichlet fractional Laplacian. J. Eur. Math. Soc. (JEMS), 12(5):1307-1329, 2010.

[14] Z.-Q. Chen, P. Kim, and R. Song. Two-sided heat kernel estimates for censored stable-like processes. Probab. Theory Related Fields, 146(3-4):361-399, 2010.

[15] Z.-Q. Chen, P. Kim, and R. Song. Dirichlet heat kernel estimates for fractional Laplacian with gradient perturbation. Ann. Probab., 40(6):2483-2538, 2012.

[16] Z.-Q. Chen, P. Kim, and R. Song. Global heat kernel estimate for relativistic stable processes in exterior open sets. J. Funct. Anal., 263(2):448-475, 2012.

[17] Z.-Q. Chen, P. Kim, and R. Song. Dirichlet heat kernel estimates for rotationally symmetric Lévy processes. Proc. Lond. Math. Soc. (3), 109(1):90-120, 2014.

[18] Z.-Q. Chen, P. Kim, and R. Song. Dirichlet heat kernel estimates for subordinate Browinan motions with Gaussian components. J. reine angew. Math., 711:111-138, 2016.

[19] Z.-Q. Chen and T. Kumagai. Heat kernel estimates for jump processes of mixed types on metric measure spaces. Probab. Theory Related Fields, 140(1-2):277-317, 2008.

[20] S. Cho. Two-sided global estimates of the Green's function of parabolic equations. Potential Anal., 25(4):387-398, 2006.

[21] K. L. Chung and Z. X. Zhao. From Brownian motion to Schrödinger's equation, volume 312 of Grundlehren der Mathematischen Wissenschaften [Fundamental Principles of Mathematical Sciences]. Springer-Verlag, Berlin, 1995.

[22] R. Cont and P. Tankov. Financial modelling with jump processes. Chapman \& Hall/CRC Financial Mathematics Series. Chapman \& Hall/CRC, Boca Raton, FL, 2004.

[23] E. B. Davies. The equivalence of certain heat kernel and Green function bounds. J. Funct. Ana, 71(1):88-103, 1987.

[24] S. N. Ethier and T. G. Kurtz. Markov processes. Characterization and convergence. Wiley Series in Probability and Mathematical Statistics: Probability and Mathematical Statistics. John Wiley \& Sons Inc., New York, 1986.

[25] B. Fristedt. Sample functions of stochastic processes with stationary, independent increments. In Advances in probability and related topics, Vol. 3, pages 241-396. Dekker, New York, 1974.

[26] M. Fukushima. On transition probabilities of symmetric strong Markov processes. J. Math. Kyoto Univ., 12:431-450, 1972.

[27] M. Fukushima. Dirichlet forms and Markov processes, volume 23 of North-Holland Mathematical Library. North-Holland Publishing Co., Amsterdam-New York; Kodansha, Ltd., Tokyo, 1980.

[28] F. Hiroshima and J. Lörinczi. Lieb-Thirring bound for Schrödinger operators with Bernstein functions of the Laplacian. Commun. Stoch. Anal., 6(4):589-602, 2012. 
[29] K. M. Hui. A Fatou theorem for the solution of the heat equation at the corner points of a cylinder. Trans. Amer. Math. Soc., 333(2):607-642, 1992.

[30] K. Kaleta, M. Kwaśnicki, and J. Małecki. One-dimensional quasi-relativistic particle in the box. Rev. Math. Phys., 25(8):1350014, 52, 2013.

[31] K. Kaleta and P. Sztonyki. Upper estimates of transition densities for stable-dominated semigroups. J. Evol. Equ., 13:633-650, 2013.

[32] G. Karch and W. A. Woyczyński. Fractal Hamilton-Jacobi-KPZ equations. Trans. Amer. Math. Soc., 360(5):2423-2442, 2008.

[33] K.-Y. Kim and P. Kim. Two-sided estimates for the transition densities of symmetric markov processes dominated by stable-like processes in $c^{1, \eta}$ open sets. Stochastic Process. Appl., 124(9):3055-3083, 2014.

[34] P. Kim, R. Song, and Z. Vondraček. Global uniform boundary Harnack principle with explicit decay rate and its application. Stochastic Process. Appl., 124(1):235-267, 2014.

[35] P. Kim, R. Song, and Z. Vondraček. Scale invariant boundary harnack principle at infinity for Feller processes. Potential Anal., pages 1-31, 2017.

[36] T. Kulczycki and M. Ryznar. Gradient estimates of harmonic functions and transition densities for Lévy processes. Trans. Amer. Math. Soc., 368(1):281-318, 2016.

[37] E. H. Lieb and R. Seiringer. The stability of matter in quantum mechanics. Cambridge University Press, Cambridge, 2010.

[38] W. E. Pruitt. The growth of random walks and Lévy processes. Ann. Probab., 9(6):948956, 1981.

[39] K.-i. Sato. Lévy processes and infinitely divisible distributions, volume 68 of Cambridge Studies in Advanced Mathematics. Cambridge University Press, Cambridge, 1999. Translated from the 1990 Japanese original, Revised by the author.

[40] R. L. Schilling and T. Uemura. On the Feller property of Dirichlet forms generated by pseudo differential operators. Tohoku Math. J. (2), 59(3):401-422, 2007.

[41] R. L. Schilling and T. Uemura. On the structure of the domain of a symmetric jump-type Dirichlet form. Publ. Res. Inst. Math. Sci., 48(1):1-20, 2012.

[42] M. L. Silverstein. Classification of coharmonic and coinvariant functions for a Lévy process. Ann. Probab., 8(3):539-575, 1980.

[43] N. T. Varopoulos. Gaussian estimates in Lipschitz domains. Canad. J. Math., 55(2):401431, 2003.

[44] T. Watanabe. The isoperimetric inequality for isotropic unimodal Lévy processes. $Z$. Wahrsch. Verw. Gebiete, 63(4):487-499, 1983.

[45] Q. S. Zhang. The boundary behavior of heat kernels of Dirichlet Laplacians. J. Differential Equations, 182(2):416-430, 2002. 


\section{Tomasz Grzywny}

Department of Pure and Applied Mathematics, Wrocław University of Science and Technology, Wybrzeże Wyspiańskiego 27, 50-370 Wrocław, Poland

E-mail: tomasz.grzywny@pwr.edu.pl

\section{Kyung-Youn Kim}

Institute of Mathematics, Academia Sinica, 6F, Astronomy-Mathematics Building, No. 1, Sec. 4, Roosevelt Road, Taipei 10617, TAIWAN

E-mail: kykim@gate.sinica.edu.tw

\section{Panki Kim}

Department of Mathematical Sciences and Research Institute of Mathematics, Seoul National University, Building 27, 1 Gwanak-ro, Gwanak-gu, Seoul 151-747, Republic of Korea

E-mail: pkim@snu.ac.kr 